
21 the Drosophila brain and many non-dFB neurons in the brain and in the Ventral Nerve

\title{
A Split-GAL4 screen identifies novel sleep-promoting neurons in the Ventral
} Nerve Cord of Drosophila

Joseph D. Jones ${ }^{1}$, Brandon L. Holder ${ }^{1}$, Kiran R. Eiken ${ }^{1}$, Alex Vogt $^{1}$, Adriana I. Velarde ${ }^{1}$, Alexandra J. Elder ${ }^{1}$, Jennifer A. McEllin ${ }^{1}$ and Stephane Dissel ${ }^{1^{*}}$

${ }^{1}$ Department of Genetics, Developmental and Evolutionary Biology, School of Biological and Chemical Sciences, University of Missouri-Kansas City, 5007 Rockhill Rd, Kansas City, MO 64110

*Correspondence to: dissels@umkc.edu

\section{Abstract}

As in the mammalian system, sleep in Drosophila is regulated by multiple brain regions. Among them, neurons projecting to the dorsal Fan-Shaped Body (dFB) have been intensively studied and the data suggest they play a critical role in sleep regulation. The 23E10-GAL4 driver is the most widely used tool to label and manipulate dFB neurons. Multiple studies have reported that activation of 23E10-GAL4 neurons promotes sleep. However, anatomical analyses revealed that 23E10-GAL4 labels 23-30 dFB neurons in 
22 Cord (VNC), the fly equivalent of the spinal cord. To better understand the role of

23 individual dFB neurons in sleep regulation, we undertook a Split-GAL4 screen to gain access to subsets of 23E10-GAL4 expressing cells. In this study, we report the discovery of two VNC cholinergic sleep-promoting neurons labeled by the 23E10-GAL4 driver.

\section{Introduction}

Understanding the neural basis of behavior is a major aspect of neurobiology. However, unequivocally assigning a behavior to a specific neuron or group of neurons is not a trivial task. The extreme complexity, diversity and connectivity of the mammalian nervous system renders this task even more daunting. To study the neural basis of a behavior, an investigator must be able to specifically manipulate a distinct group of cells and monitor the behavior of interest. Such approaches require precise genetic tools that allow for the manipulation of discrete neurons or groups of neurons. While such tools exist in mammalian systems (1), they may not be available for all the diverse types of neurons that underlie a specific behavior. It is therefore not surprising that animal models have been extensively used to untangle the neural basis of many different behaviors (2). One such model, the fruit fly Drosophila melanogaster, with its simpler nervous system and unparalleled genetic toolbox, has become a workhorse in the study of complex behaviors, such as courtship $(3,4)$, feeding $(5,6)$, and sleep $(7)$. Multiple binary systems have been developed to access and manipulate specific groups of

43 neurons in the fly nervous system: GAL4/UAS (8); LexA/LexAop (9) and; QF/QUAS

44 (10). Of these 3 systems, the GAL4/UAS system has been by far the most extensively 
used. However, the expression patterns of GAL4 drivers are often not restricted enough to clearly link a behavior to specific neurons. Importantly, refinement of GAL4 expression pattern can be achieved by employing the intersectional Split-GAL4

48 technology (11).

Sleep is a behavior that has been described in a variety of species ranging from jellyfish to humans (12). Sleep is regulated by two processes, the circadian clock which gates the occurrence of sleep, and the sleep homeostat which controls the intensity and duration of sleep, in response to prior wakefulness (13). Although the precise function of sleep still remains unknown, there is ample evidence supporting the notion that sleep is 55 required for maintaining optimal physiological and behavioral performance (14). A priori, sleep could appear to be a detrimental activity as it competes with other motivated

57 behaviors such as feeding, mating, or parenting, and renders organisms defenseless against potential predators. However, despite these negative outcomes, sleep has been maintained throughout evolution, emphasizing its essential value (15).

Over the last 20 years, multiple studies have clearly demonstrated that the mechanisms and regulation of sleep are largely conserved from flies to mammals (16). Like in the mammalian system, sleep-regulating centers are found in many areas in the Drosophila brain $(16,17)$. One such sleep-regulating center that has gathered a lot of attention comprises neurons that project to the dorsal Fan-Shaped body (dFB). The Fan-Shaped Body $(F B)$ is part of the central complex in the Drosophila brain, a region organized into multiple layers that plays a role in many functions, including locomotion control (18), 
courtship behavior (19) and memory $(20,21)$, nociception (22), visual feature recognition (23) and processing (24), social behaviors (25) and, feeding decisionmaking (26).

Previous studies demonstrated that increasing the activity of neurons contained in the C5-GAL4, 104y-GAL4, and C205-GAL4 drivers strongly promotes sleep $(27,28)$. While the expression patterns of these 3 independent drivers are broad, they show prominent overlap in neurons that project to the dFB (27). Based on these observations, the authors concluded that it is likely that the dFB plays a role in regulating sleep but could not rule out a role for neurons outside the dFB (27). In addition, further studies established that reducing the excitability of 104y-GAL4 neurons reduces sleep $(29,30)$. Highlighting the strong interaction between sleep and memory, activation of 104y-GAL4 neurons consolidates Short-Term Memory (STM) to Long-Term Memory (LTM) $(27,31)$ and restores STM to the classical memory mutant rutabaga and dunce (32). Taken together, these data point at dFB neurons as important modulator of sleep.

Further work supported a role for the dFB in sleep homeostasis by demonstrating that sleep deprivation increases the excitability of 104y-GAL4 dFB neurons (29). A follow up study proposed that increasing sleep pressure switches dFB neurons from an electrically silent to an electrically active state and that this process is regulated by dopaminergic signaling to the dFB (33) and the accumulation of mitochondrial reactive oxygen species in dFB neurons (34). The physiological properties of dFB neurons have 
led to the suggestion that dFB neurons are functionally analogous to the ventrolateral preoptic nucleus (VLPO), a key sleep-regulating center in the mammalian brain $(29,35)$.

Importantly, recent studies have used 23E10-GAL4, a more restrictive driver to manipulate and monitor dFB neurons. $(33,36)$. Several groups have demonstrated that chronic and acute activation (using thermogenetic or optogenetic methods) of 23E10GAL4 neurons increases sleep (31, 37-39). Because of its more restricted expression pattern and strong capacity to modulate sleep, 23E10-GAL4 (as it relates to sleep) is seen as a dFB specific driver by most in the scientific community. Remarkably, a recent study described an electron microscopy-based connectome of the central complex including the dFB and demonstrated that the 23E10-GAL4 driver contains 31 neurons in the brain that project to the dFB (40). Whether these $31 \mathrm{dFB}$ neurons can be considered as a functionally homogeneous group is still unclear. Interestingly, Qian et al. demonstrated that only a small subset of 23E10-GAL4 neurons express the $5 \mathrm{HT} 2 \mathrm{~b}$ serotonin receptor and that these cells regulate sleep and sleep homeostasis (37).

105 Unfortunately, this study did not investigate the role of non-5HT2b expressing 23E10-

106 GAL4 neurons in sleep regulation. In addition, whether there are non-dFB 5HT2b 107 expressing 23E10-GAL4 neurons that could potentially play a role in sleep modulation is 108 not clearly addressed in this study either. Taken together, these data suggest that 109 23E10-GAL4 dFB neurons probably constitute a functionally heterogenous group of 110 cells. 
112 Surprisingly, activation of 23E10-GAL4 expressing neurons, unlike 104y-GAL4, does

113 not consolidate STM to LTM (31). In this study, the authors demonstrated that in

114 addition to the dFB, 104y-GAL4 expresses in neurons projecting to the ventral FB (vFB)

115 and that these neurons also regulate sleep and are responsible for memory

116 consolidation (31). These data emphasize the need to use genetic tools that are as

117 specific as possible to clearly assign a role in sleep regulation and sleep function to

118 specific neurons/groups of neurons. Often, GAL4 drivers that are extensively used to

119 manipulate a specific neuronal group also express in regions outside the area of

120 interest. This makes it difficult to clearly identify the neural basis of sleep behavior.

122 With this in mind, we wanted to know which of the 31 dFB 23E10-GAL4 neurons are

123 important for sleep regulation. To do so, we employed a targeted, intersectional Split-

124 GAL4 strategy (11) focused on 23E10-GAL4 dFB neurons. Surprisingly, our data

125 demonstrates that in the 23E10-GAL4 driver, there are non-dFB sleep-promoting

126 neurons. Importantly, these cholinergic neurons are located in the Ventral Nerve Cord

127 (VNC), the fly equivalent to the spinal cord. Nevertheless, we confirm that 23E10-GAL4

128 dFB neurons regulate sleep homeostasis while 23E10-GAL4 VNC neurons do not.

129 Thus, our work reveals that there are multiple groups (at least two) of sleep-regulating

130 neurons in an extensively used "dFB-specific" GAL4 driver. These data clearly highlight

131 the need to use extremely specific tools to manipulate limited number of neurons when

132 trying to unequivocally assign a behavior to a group of cells. 
The 23E10-GAL4 driver promotes sleep and contains many non dFB neurons in the central nervous system

137 Previous studies have reported that increasing the activity of dFB neurons using the 23E10-GAL4 driver reliably increases sleep (31, 37-39). We first sought to confirm

139 these observations in our laboratory. Thus, we expressed the thermogenetic TrpA1 cation channel, which is activated by transferring flies to $31^{\circ} \mathrm{C}(41)$, in $23 \mathrm{E} 10-\mathrm{GAL} 4$ expressing neurons. Individual flies were monitored for two days at $22^{\circ} \mathrm{C}$ (baseline days, no activation), then temperature was raised to $31^{\circ} \mathrm{C}$ for $24 \mathrm{~h}$ on day 3 (activation day). A schematic of the experimental setup is provided in Figure 1A. As seen in Figure $1 \mathrm{~B}, \mathrm{C}$, raising the temperature changes the sleep profile of both parental controls and results in a loss of sleep (Figure 1E). These data are in agreement with previous reports on the effect of high temperature on sleep $(42,43)$. On the other hand, activating 23E10-GAL4 neurons strongly increases sleep (Figure 1D,E). Importantly, activation of 23E10-GAL4 neurons does not affect the amount of locomotor activity when the flies are awake (Figure 1F). Thus, we confirmed that activating 23E10-GAL4 neurons promotes sleep and that the increase in sleep is not caused by a general motor defect,

151 in agreement with previous reports using the same GAL4 driver (31, 37-39) . the field see it as a "dFB-specific" tool. However, GAL4 lines often are not that specific

155 and commonly express in neurons outside the region of interest. To clarify the 156 expression pattern of 23E10-GAL4, we expressed GFP and carefully quantified the 157 number of neurons that are GFP positive. As seen in Figure 1G, $\mathrm{H}$, there are many 
neurons labelled by 23E10-GAL4 in the brain and in the VNC (See also Video 1 and Video 2). Importantly, many of these neurons are not dFB neurons. Our analysis revealed that there are more than 50 neurons in the brain labelled by 23E10-GAL4, only half of which are dFB neurons (Table1). In addition, 23E10-GAL4 labels about 18 neurons in the VNC (Table1). Interestingly, we note that while the electron microscopy study discussed above identified 31 dFB 23E10-GAL4 neurons (40), we do not systematically observe 31 neurons in every brain. This discrepancy is probably due to the different methodologies used in the two studies. We suspect that some 23E10GAL4 dFB neurons may be only weakly expressing GAL4 and are therefore not always visible using confocal microscopy. However, based on this expression pattern, we conclude that it is impossible to unequivocally assign a role in sleep promotion to 23E10-GAL4 dFB neurons.

\section{The 23E10-GAL4 driver contains sleep-promoting neurons that are not dFB} neurons

To shed light on the role of individual 23E10-GAL4 expressing neurons in sleep regulation, we employed a Split-GAL4 strategy (11) to access a reduced number of 23E10-GAL4 expressing cells. In designing this screen, our primary region of interest in the brain was the dFB, a choice dictated by the number of studies supporting a role for this structure in sleep regulation $(27-30,33,34,37,44,45)$. Central to the Split-GAL4 technology is the fact that the functional GAL4 transcription factor can be separated into two non-functional fragments, a GAL4 DNA-binding domain (DBD) and an activation domain (AD). Different enhancers are used to express these two fragments and the 
181 functional GAL4 transcription factor is reconstituted only in the cells in which both

182 enhancers are active (11).

183 We obtained 22 different AD lines based on their associated GAL4 line expressing in 184 the dFB, as observed using the Janelia Flylight website $(36,46)$. Since the goal of our 185 screen was to identify the contribution of individual 23E10 dFB neurons in sleep 186 regulation, we designed a targeted approach by combining these individual $A D$ lines to a 23E10-DBD line $(46,47)$, thus creating 22 new Split-GAL4 lines named FBS for FanShaped Body Spplits lines (See Table2 for a description of these lines). These newly created lines were screened behaviorally for their ability to modulate sleep and anatomically to identify their expression patterns. To do so, each individual Split-GAL4 FBS line was crossed to a line expressing both the thermogenetic TrpA1 cation channel and a mCD8GFP construct. Flies were maintained at $22^{\circ} \mathrm{C}$ for 2 days before raising the temperature to $31^{\circ} \mathrm{C}$ at the beginning of day 3 for a duration of $24 \mathrm{~h}$. Temperature was brought down to $22^{\circ} \mathrm{C}$ on day 4 (Figure $2 \mathrm{~A}$ ). For each individual fly, we calculated the 195 percentage of total sleep change between activation day (day3) and baseline day 196 (day2) (Figure 2A). As a control for this screen, we used an enhancerless AD construct 197 (created in the vector used to make all the AD lines) combined with 23E10-DBD, since it 198 is the common element in all the Split-GAL4 lines screened in this experiment. As seen 199 in Figure $2 \mathrm{~B}, \mathrm{D}$, control female flies show a reduction in sleep upon transferring to $31^{\circ} \mathrm{C}$, 200 an effect particularly evident during the night. These data are in agreement with 201 previous studies documenting that changes in temperature modulate sleep architecture 202 and that high temperature disturbs sleep at night $(42,43,48,49)$. However, acute 203 activation of the neurons contained in 9 of the 22 FBS lines led to significant increases 
204 in percentage sleep change in female flies (FBS4, FBS5, FBS42, FBS45, FBS53,

205

206

207

208

209

210

211

212

213

214

215

216

217

218

219

220

221

222

223

224

225

226

FBS68, FBS72, FBS81 and FBS84), when compared with controls. Individual sleep

traces for all 22 FBS lines are shown in Figure 2E,F,G,H and Figure 2-figure

supplement 1. Importantly, analysis of activity counts during awake time reveals that increases in sleep are not caused by a reduction in locomotor activity (Figure 2C). On

the contrary, 3 of the strongly sleep-inducing lines even show an increase in waking activity upon neuronal activation (Figure 2C). This increase is likely explained by the need for a fly to perform tasks that are mutually exclusive to sleep in a reduced amount of waking time. While increases in total sleep are indicative of increase sleep quantity, this measurement does not provide information about sleep quality or sleep depth. To assess whether sleep quality is modulated by FBS lines, we analyzed sleep consolidation during the day and night in these flies. As seen in Figure 2-figure supplement 2A, upon neuronal activation, daytime sleep bout duration is significantly increased in 8 out of the 9 sleep-promoting lines (FBS4, FBS5, FBS42, FBS45, FBS53, FBS68, FBS72 and FBS84), while it is unaffected in FBS81. Notably, increased sleep bout duration is believed to be an indication of increased sleep depth (50). Thus, these data indicate that activating neurons contained in these 8 FBS lines not only increases sleep level but probably also increases sleep depth. Interestingly, one of the lines that did not show an increase in total sleep, has a significant increase in daytime sleep bout duration (FBS28). During the nighttime, the effect of high temperature on sleep is obvious as most lines, including the control, display a significant decrease in sleep bout duration when raising the temperature to $31^{\circ} \mathrm{C}$ (Figure 2-figure supplement $2 \mathrm{~B}$ ). Only 6 of the 9 sleep-promoting lines (FBS4, FBS5, FBS42, FBS45, FBS53 and FBS68) 
227 maintain similar nighttime sleep bout durations before and during neuronal activation.

228 Importantly, in these 6 lines nighttime sleep bout duration is significantly increased at

$22931^{\circ} \mathrm{C}$ when compared to control flies (Figure 2-figure supplement 2B). These data

230 indicate that most sleep-promoting Split-GAL4 lines increase daytime sleep

231 consolidation and suggest that they could also increase nighttime sleep consolidation.

232 However, because of the clear effect of high temperature on nighttime sleep, this claim

233 requires further validation.

235 In addition, since sleep in Drosophila is sexually dimorphic (51-53), we systematically

236 assessed male flies in our experiments. As seen in Figure 2-figure supplement 3, we

237 obtained almost identical behavioral data when analyzing male flies using our

238 thermogenetic activation approach. Thus, we conclude that we have identified 9 FBS

239 lines that promote sleep when thermogenetically activated in males and females.

241 Having identified 9 different Split-GAL4 lines that significantly increased sleep when

242 activated, we sought to identify the neurons that are contained within these lines.

243 Because the goal of our screen was to identify sleep-regulating dFB neurons in the

244 23E10-GAL4 pattern of expression, we expected that most of our FBS lines, sleep-

245 promoting or not, would express in dFB cells. This is indeed the case as 20 out of the

24622 FBS lines express in some dFB neurons (Figure 2, Figure 2-figure supplement 1,

247 Table 2). The two exceptions to this are FBS25 (expresses in no neurons at all) and

248 FBS5 as discussed below. Regarding sleep-promoting FBS lines, 8 out of 9 have some

249 expression in the dFB. However, and surprisingly, one of the strongly sleep-promoting 
250 lines, FBS5, does not express in any dFB neurons at all (Figure 2F, Video 3, Video 4).

251 Rather, FBS5 strongly expresses in two clusters of 4-5 neurons located in the anterior

252 ventrolateral protocerebrum and in 4 VNC cells located in the metathoracic ganglion. A

253 close examination of the anatomy of these 4 VNC neurons indicate that they have

254 processes in the brain. Two of these neurons show a very specific pattern of expression

255 with brain processes located in the superior medial protocerebrum. Because of their

256 characteristic look, we named these processes "bowtie". These processes are

257 highlighted in the right panel in Figure 2F (see also Video 3). FBS5 additionally labels

258 two neurons in the VNC whose cell bodies are located very close to the "bowtie"

259 neurons. These neurons have characteristic processes in each leg ganglion (yellow

260 arrows in Figure 2F-VNC image). Based on their anatomy, we hypothesize that these

261 cells could be Taste Projection Neurons 1 (TPN1) that receive inputs from sweet

262 gustatory receptor leg neurons (GRNs) and convey this information to the brain in the

263 subesophageal zone (SEZ) (54). We observed these putative TPN1 neurons in 14 out

264 of the 22 FBS lines. Importantly, since all the neurons in FBS5 are part of the 23E10-

265 GAL4 expression pattern, these data indicate that this driver contains non-dFB sleep-

266 promoting cells.

268 Most other sleep-promoting line expression patterns are very discrete with only two

269 major areas of expression; dFB neurons, and the metathoracic ganglion region

270 containing the two "bowtie" neurons and TPN1-like cells (FBS42, FBS45, FBS53,

271 FBS68, FBS72, FBS81 and FBS84) (Figure 2G,H; Figure 2-figure supplement 1;

272 Table2). Some of these FBS lines show minimal amount of expression elsewhere in the 
273 brain, but there is no consistency in these additionally labeled cells between different

274 lines. Of note, FBS4 expresses in 18-23 dFB neurons (Figure 2E, Table 2) and no other

275 cells in the brain or VNC. Considering that 23E10-GAL4 reliably labels 23-30 dFB

276 neurons in our confocal microscopy experiments (Table1), we conclude that FBS4

277 expresses in $75-80 \%$ of $23 E 10$ dFB neurons. Because of its dFB specific expression

278 pattern and its ability to increase sleep when thermogenetically activated, we suspect

279 that FBS4 probably contains neuron(s) that are regulating sleep. However, we also note

280 that the sleep increase triggered by FBS4 thermogenetic activation is not as striking as

281 when activating other strongly sleep-promoting lines like FBS5, FBS53 or FBS68

282 (Figure 2B for females and Figure 2-figure supplement 3A for males).

284 One particularly interesting line is FBS45. FBS45 is strongly sleep-promoting and is 285 expressed in 21-32 dFB neurons, indicating that most if not all 23E10 dFB neurons are 286 present in FBS45 (Figure 2G, Table2). However, FBS 45 also expresses in the two 287 "bowtie" neurons. Importantly, FBS45 does not label any other neurons in the brain and 288 VNC.

290 When combining behavioral and anatomical data, it appears that the numbers of dFB 291 neurons contained within a specific FBS line cannot be predictive of whether the line 292 can promote sleep upon neuronal activation. For example, FBS68 strongly promotes 293 sleep and expresses in only 3-6 dFB neurons (Figure 2H, Table 2), while FBS60 294 contains 22-27 dFB neurons and does not promote sleep (Figure 2-figure supplement 295 1L, Table 2). 
296 Remarkably, a careful examination of expression patterns reveals that many FBS lines

297 appear to express in the "bowtie" VNC neurons. Note that the brain processes of these

298 cells are located extremely close to the axonal projections of dFB neurons, making the

299 visualization of these VNC projections sometimes difficult, depending on the strength of

300 dFB projection's staining. Nevertheless, these VNC "bowtie" neurons are present in 17

301 out of 22 FBS lines (See Figure 2 and Figure 2-figure supplement 1 VNC "bowtie"

302 processes). Importantly, we can see these neurons and their processes in all sleep-

303 promoting lines with the notable exception of FBS4 (Figure 2, Figure 2-figure

304 supplement 1, Table 2), as discussed above. Upon examination of the literature, we

305 found that neurons with similar projections are frequently seen in many Janelia GAL4

306 lines (55). This study reports that these neurons (referred to as "sparse T" in this work)

307 and their processes are seen in up to $60 \%$ of all Janelia GAL4 lines. The fact that these

308 "bowtie" neurons are frequently present in many GAL4 lines probably explains why we

309 see them in the vast majority of our FBS lines (18 out of 22 or $82 \%$ of them).

311 In conclusion, our thermogenetic screen and anatomical analysis identified 9 sleep-

312 promoting lines with diverse patterns of expression. While the identity of the neurons

313 responsible for sleep promotion in these different lines is not clear at this stage, our

314 analysis indicates that there are multiple groups of sleep-promoting neurons in the

315 23E10-GAL4 pattern of expression; one of which appears to be part of the dFB (FBS4)

316 while the other is non-dFB based (FBS5).

\section{Optogenetic confirmation of sleep promoting FBS lines}


319 Because of the strong effect that temperature has on sleep, we sought to confirm our thermogenetic findings using an alternative way to manipulate FBS lines. The logic

321 behind our thinking is that it may be difficult to fully describe and characterize the sleep

322 behaviors of our FBS lines when temperature itself has such a profound effect on sleep.

323 Furthermore, a recent study demonstrated that some of the effects of high temperature

324 on sleep are mediated by GABAergic transmission on dFB neurons (49). Taken

325 together, these data suggest that thermogenetic manipulations may not be optimal to

326 conduct our screen. We thus undertook an optogenetic screen using CsChrimson (56).

327 Our optogenetic experimental setup is described in Figure 3A. Notably, for neurons to

328 be activated when expressing CsChrimson, flies need to be fed all trans-retinal and the

329 neurons must be stimulated with a 627nm LED light source (56). Thus, optogenetic

330 approaches provide a much better control on the timing and parameters of activation.

331 Nonetheless, optogenetic activation gave results that are mostly identical to the

332 thermogenetic approach. That is, most sleep-promoting lines identified using TrpA1 also

333 induce sleep when activated with CsChrimson in retinal-fed flies (FBS5, FBS42, FBS45,

334 FBS53, FBS68 and FBS72) (Figure 3B for female data). Notable differences are FBS4,

335 FBS81 and FBS84 which do not promote sleep when optogenetically activated in

336 female flies. However, activating FBS81 and FBS84 significantly increases sleep in

337 retinal-fed male flies (Figure 3-figure supplement 2A). Importantly, unlike FBS81 and

338 FBS84, activation of FBS4 neurons does not increase sleep in retinal-fed males (Figure

339 3-figure supplement 2A). Thus, there is a clear discrepancy for FBS4 comparing

340 thermogenetic and optogenetic activations. Another difference between the two

341 activation approaches is FBS70 that is sleep-promoting using CsChrimson in retinal-fed 
342 female flies while it was not using TrpA1 (Figure 3B). Surprisingly, retinal-fed

343 FBS70>UAS-CsChrimson males do not increase sleep upon LED stimulation,

344 potentially revealing a sexually dimorphic phenomenon (Figure 3-figure supplement 2A).

345 Importantly, assessment of locomotor activity during waking period reveals that

346 optogenetic activation does not decrease waking activity in long sleeping retinal-fed

347 females (Figure 3C) and males (Figure 3-figure supplement 2B), ruling out the

348 possibility that locomotor defects are the underlying cause of the sleep phenotypes

349 observed. Examination of sleep bout duration in retinal-fed flies indicates that most

350 sleep-promoting FBS lines increase sleep consolidation during the day (Figure 3D for

351 females and Figure 3-figure supplement $2 \mathrm{C}$ for males) and during the night (Figure 3E

352 for females and Figure 3-figure supplement 2D for males). These data indicate that

353 optogenetic activation increases sleep quantity and probably sleep quality (sleep depth)

354 in many of the FBS lines first identified using our thermogenetic approaches.

355 Interestingly, some FBS lines that did not increase total sleep upon optogenetic

356 activation increased daytime sleep bout duration: FBS57 and FBS60 females (Figure

357 3D) and FBS33, FBS35, FBS64 and FBS87 males (Figure 3-figure supplement 2C).

358 These data suggest that these lines may also express in sleep-regulating neurons.

360 Importantly, analysis of sleep parameters in vehicle-fed flies demonstrate that the sleep

361 phenotypes we observed are specific to retinal-fed and LED stimulated flies (Figure 3-

362 figure supplement 1 for females and Figure 3-figure supplement 3 for males). 
364 In conclusion, our thermogenetic and optogenetic neuronal activation revealed several

365 strongly sleep-promoting lines. When looking at their expression patterns, our data

366 clearly demonstrate that 23E10 GAL4 contains at least two different sleep-promoting

367 regions, one that is part of the $\mathrm{dFB}$ and one that is not.

The 23E10-GAL4 driver contains sleep-promoting neurons that are not located in the brain

371 To reveal the identity of the non-dFB 23E10 sleep-promoting neurons, we undertook a second screen creating additional Split-GAL4 lines focusing on neurons that are present in FBS5. To facilitate this task, we used Color Depth MIP mask search, a software plug374 in for Fiji (57). This approach identified several AD lines (containing neurons of interest) 375 that we combined with our 23E10-DBD stock. An example of such a line is shown in 376 Figure 4A-D. This Split-GAL4 line (VT020742-AD;23E10-DBD) expresses only in 4 VNC 377 neurons (Figure 4C). Projections of 2 of these are clearly "bowtie" like (gray arrows in 378 Figure 4A-B). Projections of the other two appear to be identical to TPN1 neurons that 379 have been previously described (yellow arrows in Figure 4A-D). Remarkably, VT020742-AD;23E10-DBD does not express in any other neurons in the brain and 381 VNC. To confirm the presence of TPN1 neurons in this Split-GAL4 line we expressed 382 GFP using a TPN1 specific driver, 30A08-LexA (54) while simultaneously expressing 383 RFP using VT020742-AD;23E10-DBD. As seen in Figure 4-figure supplement 4A, there 384 is clear GFP and RFP co-expression in two out of the four VT020742-AD;23E10-DBD 385 neurons (yellow arrows). In addition, we also see co-expression of GFP and RFP in the 386 processes that go into each leg ganglion, but only RFP expression in the "bowtie" 
387 processes. From these data we conclude that VT020742-AD;23E10-DBD labels 4

388 neurons in the metathoracic ganglion of the VNC: 2 "bowtie" neurons and 2 TPN1 cells.

389 Optogenetic activation of these 4 neurons strongly increases sleep in retinal-fed

390 females (Figure 4K) and males (Figure 4-figure supplement 2A). In addition, daytime

391 and nighttime sleep bout duration are significantly increased in retinal-fed VT020742-

392 AD;23E10-DBD>UAS-CsChrimson flies (Figure 4M-N for females and Figure 4-figure

393 supplement 2C-D for males). Importantly, analysis of waking activity reveals that the

394 sleep increases are not caused by a deficit in locomotor activity (Figure 4L for females

395 and Figure 4-figure supplement 2B for males).

396

397 We then wanted to find out whether TPN1 neurons are the cells involved in sleep

398 promotion in VT020742-AD;23E10-DBD. We thus created a 30A08-AD;23E10-DBD

399 Split-GAL4 line. This line labels only the two TPN1 neurons and no other cells in the

400 brain and VNC (Figure 4E-F). Importantly, optogenetic activation of 30A08-AD;23E10-

401 DBD neurons has no effect on sleep (Figure 4K-N and Figure 4-figure supplement 2A-

402 D), indicating that the sleep-promoting capacity of VT020742-AD;23E10-DBD most

403 likely lies within the two "bowtie" neurons. To further rule out an involvement of TPN1

404 neurons in sleep promotion, we adopted the Split-GAL4 repressor Killer Zipper (KZip+)

405 technology which inhibits an AD and DBD from forming a functional GAL4 (58).

406 Expressing KZip + with 30A08-LexA while expressing GFP with VT020742-AD;23E10-

407 DBD, reduces the number of GFP positive cells from 4 to 2 (Figure 4-figure supplement

408 4B). Only the two "bowtie" neurons can be seen in this combination. Thus, we can

409 effectively subtract the TPN1 neurons from the VT020742-AD;23E10-DBD pattern of 
410

411

412

413

414

415

416

417

418

419

420

421

422

423

424

425

426

427

428

429

430

431

432

expression. Optogenetic activation of the remaining "bowtie" neurons (in retinal-fed VT020742-AD;23E10-DBD>UAS-CsChrimson, 30A08-LexA>LexAop2-KZip+ flies) still promotes sleep (Figure 4-figure supplement 4C). Taken together these data indicate that the two "bowtie" neurons in VT020742-AD;23E10-DBD (and 23E10-GAL4) increase sleep when activated and that TPN1 neurons are not involved in sleep promotion.

To further refine our analysis, we constructed an additional Split-GAL4 line (VT013602$A D ; 23 E 10-D B D$ ) that only expresses in the two "bowtie" like VNC neurons (gray arrows in Figure 4G-J, Video 5, Video 6). Optogenetic activation of retinal-fed VT013602AD;23E10-DBD neurons strongly promotes sleep (Figure 4K and Figure 4-figure supplement 2A for male data) and increases daytime (Figure 4M and Figure 4-figure supplement 2C) and nighttime bout duration (Figure 4N and Figure 4-figure supplement 2D). Again, increases in sleep are not caused by abnormal locomotor activity (Figure 4L and Figure 4-figure supplement 2B). Importantly, analysis of sleep parameters in vehicle-fed flies reveal sleep phenotypes observed are specific to activation of VT013602-AD; 23E10-DBD neurons (Figure 4-figure supplement 1 and Figure 4-figure supplement 3). Since activating the two "bowtie" neurons increases daytime and nighttime sleep bout duration, we hypothesize that this manipulation increases sleep quality, namely sleep depth. To investigate this possibility, we measured arousal thresholds in VT013602-AD;23E10-DBD>UAS-CsChrimson flies. As seen in Figure 4figure supplement 5 , the proportion of retinal-fed flies awakened by mild mechanical perturbations ( 1 and 2 ) is significantly reduced in LED stimulated (LED ON, activated) individuals compared with non-activated flies (LED OFF). Thus, these data demonstrate 
433 that when activated, the two "bowtie" neurons increase sleep quantity and sleep depth

434 as indicated by an increase in arousal threshold. Importantly, at the highest perturbation

435 level (4), retinal-fed LED activated VT013602-AD;23E10-DBD>UAS-CsChrimson flies

436 respond to the stimulus similarly to controls, indicating that the sleep that is induced by

437 activation of these neurons is reversible.

438

439 To assess whether the "bowtie" neurons seen in VT020742-AD;23E10-DBD and

440 VT013602-AD;23E10-DBD are the same, we constructed a line that carries both

441 VT020742-AD and VT013602-AD as well as 23E10-DBD. Expressing GFP with this line

442 labels only 4 VNC neurons, 2 "bowtie" cells and 2 TPN1 (Figure 4-figure supplement

443 4D). If the "bowtie" neurons were different in VT020742-AD;23E10-DBD and VT013602-

$444 \quad A D ; 23 E 10-D B D$ we would expect to see 6 cells. Since this is not the case, we can

445 conclude that the 2 "bowtie" neurons observed in VT020742-AD;23E10-DBD and

446 VT013602-AD;23E10-DBD are the same. To provide functional support to this finding,

447 we created an additional Split-GAL4 line, VT013602-AD;VT020742-DBD. This Split-

448 GAL4 line labels 2 "bowtie" cells in the VNC with typical processes in the brain (gray

449 arrows in Figure 4-figure supplement 4E) and promotes sleep when activated

450 thermogenetically (Figure 4-figure supplement 4F-H). Taken together, these data

451 demonstrate that we have unequivocally identified two novel sleep-promoting neurons

452 located in the VNC. Importantly, these cells are part of the 23E10-GAL4 expression

453 pattern. We will from now on refer to these neurons as VNC-SP (for VNC Sleep-

454 Promoting). 


\section{Silencing VNC-SP neurons reduces sleep}

457 Having demonstrated that activation of VNC-SP neurons increases sleep duration and

458 sleep depth, we wondered whether these cells could modulate sleep bi-directionally.

459 Thus we performed experiments to silence the activity of VNC-SP neurons either

460 chronically, by expressing the inward rectifying potassium channel Kir2.1 (59) or acutely

461 by using the temperature sensitive Shits1 construct (60). As seen in Figure 5A-B, total

462 sleep is significantly reduced when expressing Kir2.1 in VNC-SP neurons in female

463 flies. This reduction in sleep is accompanied by a reduction of both daytime (Figure 5C)

464 and nighttime sleep bout duration (Figure 5D), suggesting a defect in sleep

465 consolidation. Importantly, analysis of activity counts during waking time reveals that the

466 decrease in sleep is not due to hyperactivity (Figure 5E). We obtained identical

467 behavioral results when expressing Kir2.1 in VNC-SP neurons of male flies (Figure 5F-

468 J). Thus, chronic silencing of VNC-SP neurons decreases sleep and disrupts sleep

469 consolidation.

470

471 To confirm the findings obtained using chronic silencing we decided to employ an acute

472 silencing protocol by expressing Shits1 (60) in VNC-SP neurons. Notably, this approach

473 relies on increasing temperature to silence neurons. Because of the previously reported

474 effect of temperature on sleep, it is not surprising to see that the shift in temperature

475 reduces sleep in control flies (Figure $5 \mathrm{~K}$ for females and Figure $5 \mathrm{~N}$ for males). Acute

476 silencing of VNC-SP neurons also reduces sleep, an effect particularly striking at night

477 (Figure 5L and Figure 5M). Importantly, nighttime sleep is significantly more reduced in

$478 \quad$ VNC-SP $>$ Shits1 flies than in controls (Figure 5M and Figure 5P). 
479 Taken together, our chronic and acute silencing data demonstrate that VNC-SP neurons positively regulate sleep. Importantly, these results further reinforce our

481 findings using optogenetic and thermogenetic activation experiments.

\section{VNC-SP neurons express and use acetylcholine to modulate sleep}

484 Having demonstrated that VNC-SP neurons modulate sleep bi-directionally, we sought to identify the neurotransmitter(s) used by these cells. A previous study suggested that

486 most neurons in the VNC use one of three neurotransmitters: GABA, Glutamate or Acetylcholine (61). With this in mind, we expressed GFP in VNC-SP neurons and incubated them with antibodies to GABA, choline acetyltransferase (ChAT), the enzyme necessary to produce Acetylcholine and the vesicular glutamate transporter (VGlut). As seen in Figure 6A, we observed clear co-labelling of GFP and ChAT in VNC-SP neurons. To further confirm that VNC-SP neurons express ChAT, we employed a combinatorial approach expressing RFP in VNC-SP cells and GFP driven by a ChATLexA (62) construct that is inserted within the endogenous ChAT locus. This construct ensures that the expression of LexA is controlled by ChAT regulatory sequences and should reflect transcriptional activity at the endogenous ChAT locus. As seen in Figure

496 6-figure supplement 1A, we observe clear co-labelling of GFP and RFP in VNC-SP 497 neurons (gray arrow). In addition, we employed a similar approach using 23E10-GAL4

498 to drive expression of RFP. As seen in Figure 6-figure supplement 1B, we observe clear 499 co-labelling of GFP and RFP in two neurons in the metathoracic ganglion of the VNC

500 (gray arrows). The evidence clearly suggests that these two cells are the VNC-SP 
502 per brain hemisphere are co-labelled by 23E10-GAL4 and ChAT-LexA construct (Figure

503 6-figure supplement 1B, bottom panels, red arrows). Taken together, these data

504 indicate that VNC-SP neurons and some 23E10-GAL4 dFB neurons are cholinergic.

505

506 Importantly, we did not detect any co-labelling between GFP and VGlut (Figure 6B) or

507 GABA (Figure 6C) in these experiments. To find out whether VNC-SP neurons express

508 any additional neurotransmitters we also performed experiments using antibodies to

509 serotonin (5HT, Figure 6D), tyrosine hydroxylase (TH, Figure 6E), the key enzyme in

510 the synthesis of dopamine, and tyrosine decarboxylase 2 (Tdc2, Figure 6F), an enzyme

511 involved in the synthesis of octopamine. We did not observe any co-labelling with any of

512 these 3 antibodies.

514 These data indicate that VNC-SP neurons are cholinergic but they do not provide any

515 information as to whether cholinergic transmission is needed for the sleep modulating

516 role of VNC-SP neurons. To address this question, we sought to disrupt cholinergic

517 transmission in VNC-SP neurons using an RNAi approach. Thus, we obtained two

518 independent RNAi lines for ChAT. We first sought to validate them by crossing them to

519 the pan-neuronal elav-GAL4 driver. One of the lines (60028) led to lethality when

520 crossed to elav-GAL4, indicative of a significative reduction in ChAT levels. The second

521 line (25856) gave rise to progeny suggesting it is not very efficient. Note that a similar

522 approach was used to validate ChAT RNAi constructs in other studies (63). However, to

523 further confirm the efficiency of these ChAT RNAi constructs we performed a qPCR

524 analysis on flies expressing ChAT RNAi (60028) specifically in the eyes using a GMR- 
525 GAL4 driver. Figure 6-figure supplement 1C demonstrates that ChAT mRNA levels are significantly reduced (about $20 \%$ ) in the eyes of GMR-GAL4>ChAT RNAi (60028) flies compared with controls. Notably, ChAT mRNA levels are unaffected in the body of

528 these flies, illustrating the efficiency and specificity of the ChAT RNAi line. Importantly, qPCR analysis of the second ChAT RNAi line (25856) showed no significant reduction

530 in ChAT levels, further demonstrating its inefficiency (data not shown). Based on this 531 data, we decided to only employ one ChAT RNAi (60028) in our behavioral studies.

As seen in Figure 6-figure supplement 1D-E, ChAT levels as measured by immunocytochemistry are significantly reduced when expressing ChAT RNAi in VNC-

535 SP neurons. Behaviorally, reducing ChAT levels in VNC-SP neurons significantly

536 reduces sleep (Figure 6G-H) and, daytime (Figure 6I) and nighttime (Figure 6J) sleep

537 bout duration in female flies. Importantly, the reduction in sleep is not due to

538 hyperactivity (Figure 6K). We obtained similar behavioral data when looking at males

539 (Figure 6-figure supplement 1F-I). Taken together, our data indicate that cholinergic

540 transmission is involved in regulating the sleep modulating effect of VNC-SP neurons.

541 We also note that the behavioral effects of reducing ChAT levels and silencing VNC-SP

542 neurons are consistent with each other, suggesting that in the absence of ChAT, the

543 ability of VNC-SP neurons to positively regulate sleep is seriously diminished. To further

544 interrogate the necessity of cholinergic transmission for VNC-SP neurons to promote

545 sleep, we activated VNC-SP neurons optogenetically, while simultaneously expressing

546 ChAT RNAi in these cells. As seen in Figure 6L, expression of CsChrimson and a RNAi

547 line targeting GFP (control RNAi) does not prevent sleep increase in retinal-fed flies. 
548 However, expressing ChAT RNAi completely abolishes the sleep promotion triggered by

549 activation of VNC-SP neurons. In conclusion, our data clearly demonstrate that VNC-SP

550 neurons are cholinergic and that disruption of cholinergic transmission abolishes the

551 sleep-promoting role of these cells.

552

553 To further confirm that the sleep phenotypes we observe are due to VNC-SP neurons,

554 we optogenetically activated VNC-SP cells while expressing the Split-GAL4 repressor

$555 \mathrm{KZip}+(58)$ under the control of a ChAT-LexA driver. As seen in Figure 6-figure

556 supplement 1J, expressing the KZip+ repressor in ChAT expressing neurons blocks the

557 sleep induction caused by activation of VNC-SP neurons. These data strongly suggest

558 that the KZip+ is expressed in VNC-SP neurons and actively represses the binding of

559 the GAL4-AD and GAL4-DBD constructs. To visually confirm repression of GAL4

560 activity, we expressed GFP in VNC-SP neurons and the KZip+ repressor in ChAT-LexA

561 cells. As seen in Figure 6-figure supplement 1K, expressing the KZip+ repressor in

562 ChAT expressing neurons completely abolishes the expression of GFP seen in VNC-SP

563 neurons. Taken together, these data confirm that the sleep phenotypes we observe are

564 due to activation of VNC-SP neurons and further demonstrate that these cells are

565 cholinergic.

566

567 Since VNC-SP neurons are observed in 18 of our 22 FBS lines, and in most sleep568 promoting lines (except for FBS4), we wondered whether VNC-SP neurons are the cells 569 responsible for the sleep increase in all our lines. We decided to focus on FBS45, since 570 it expresses in most if not all 23E10-GAL4 dFB neurons (Table 2) and in the two VNC- 
571 SP neurons. Importantly, FBS45 is one of the strongest sleep-promoting lines we have

572 studied in this work. Our question was the following: Is the sleep increase caused by

573 activation of FBS45 due to VNC-SP neurons, dFB neurons, or both group of cells? To

574 answer this question, we expressed UAS-CsChrimson in FBS45 neurons while

575 simultaneously driving the expression of the KZip+ repressor in ChAT-LexA cells. We

576 hypothesize that this manipulation should repress GAL4 formation specifically in VNC-

577 SP neurons. However, since we have reported that ChAT-LexA also expresses in some

578 23E10-GAL4 dFB neurons (Figure 6-figure supplement 1B), our manipulation may

579 repress GAL4 in a few dFB neurons. As seen in Figure 6-figure supplement 1L,

580 expressing the KZip+ repressor in ChAT-LexA prevents retinal-fed FBS45>UAS-

581 CsChrimson flies from increasing sleep when activated. These data indicate that the

582 neurons responsible for the sleep increase when activating FBS45 cells are cholinergic

583 and that expressing CsChrimson only in FBS45 neurons that are not targeted by ChAT-

584 LexA (non-cholinergic) cannot promote sleep. We then visually assessed the extent of

585 ChAT-LexA driven KZip+ repression in FBS45 neurons by expressing UAS-GFP. As

586 seen in Figure 6-figure supplement 1M, expressing the KZip+ repressor in ChAT-LexA

587 completely abolishes GFP expression in VNC-SP neurons in FBS45>UAS-GFP; ChAT-

588 LexA>LexAop2-KZip+ flies. However, as we expected based on our previous data,

589 ChAT-LexA>LexAop2-KZip+ consistently removes GFP expression in 4-6 dFB neurons

590 as well. Taken together, these data suggest that the sleep-promoting capacity of FBS45

591 relies on cholinergic neurons. While we strongly believe that the cells responsible are

592 VNC-SP neurons, we cannot completely exclude a role for one of the ChAT expressing 
23E10-GAL4 dFB neurons. Further work will help clarify the role of cholinergic dFB neurons in sleep regulation.

Since the VNC receives and integrates sensory inputs from the periphery and sends

596 this information to the brain (64-66), we wondered whether VNC-SP neurons could

597 similarly send sleep-relevant signals to the brain. The location of VNC-SP neurons in

598 the VNC, and their processes in the brain suggests that this is a likely possibility. To find 599 out, we expressed the dendritic marker DenMark (67) in conjunction with the 600 presynaptic marker synaptotagmin-GFP (syt.eGFP) (68) in VNC-SP neurons. Our data 601 indicate that VNC-SP neurons have mostly postsynaptic sites in the VNC, as expected if 602 these cells receive sensory information from the periphery (Figure 6-figure supplement 603 2, middle panels). However, and surprisingly, the brain processes of these cells are 604 labeled with both the presynaptic and postsynaptic markers (Figure 6-figure supplement 6052 , top panels). This suggests that VNC-SP neurons are well positioned to receive 606 sensory inputs from the periphery in the VNC and that they are sending this information

607 to the brain. However, since the brain processes of VNC-SP neurons are also harboring 608 postsynaptic sites, it is highly likely that they also get inputs in the brain. Nevertheless, our data suggest that VNC-SP neurons send sleep-relevant signals to the brain.

612 Our data unequivocally demonstrate that VNC-SP neurons, which are part of the 613 23E10-GAL4 pattern of expression, are sleep-promoting. Since 23E10-GAL4 is widely 614 seen as dFB specific driver when it comes to sleep regulation, the identification of VNC615 SP neurons is a major finding that impacts our current views about the role of the dFB in 
sleep regulation. Our data shows that all but one FBS sleep-promoting lines contain VNC-SP neurons. The only sleep-promoting line that is dFB specific (FBS4) only mildly promotes sleep (when compared with other lines) in our thermogenetic screen but does not using optogenetic. These data raise important questions about the role of the dFB in sleep regulation and sleep-promotion.

To try to address these questions we went back to the 23E10-GAL4 driver and its 23E10-LexA counterpart (69). First, we wanted to check whether 23E10-GAL4 and 23E10-LexA express in the same number of neurons. This is an important control since it was reported that often for a given enhancer sequence, the LexA expression pattern does not fully recapitulate the GAL4 expression pattern (69). We created a line expressing GFP under the control of 23E10-LexA and RFP under the control of 23E10GAL4. As seen in Figure 7A-B, the 23E10-LexA driver does not fully recapitulate the 23E10-GAL4 pattern of expression in the dFB. Some cells are clearly co-labelled by GFP and RFP (yellow arrows) but some other dFB neurons only express RFP (gray arrow). Importantly, we did not observe any dFB cells (or any other cell in the brain) that are only labeled by 23E10-LexA. These data indicate that, like many other LexA drivers, 23E10-LexA only expresses in a subset of the 23E10-GAL4 pattern of expression. We then turned our attention to the metathoracic ganglion of the VNC. As seen in Figure 7C, 23E10-LexA labels the two TPN1 neurons (yellow arrows) while 23E10-GAL4 labels the two TPN1 cells as well as the two VNC-SP neurons (gray arrows). The processes of TPN1 neurons are highlighted in Figure 7E and those from VNC-SP in Figure 7D. We see clear co-labelling of TPN1 processes by 23E10-LexA and 23E10-GAL4, but VNC- 
SP processes are only labeled by 23E10-GAL4. Taken together, we conclude that 23E10-LexA only expresses in a subset of 23E10-GAL4 neurons and that 23E10-LexA does not express in VNC-SP neurons.

We then wondered whether 23E10-LexA could promote sleep when activated

644 optogenetically. As seen in Figure 7F, optogenetic activation of retinal-fed 23E10changes in sleeping levels. However, optogenetic activation of retinal-fed 23E10LexA>LexAop2-CsChrimson has no effect on sleep (Figure 7G). The stark difference in behavior seen when activating 23E10-GAL4 and 23E10-LexA could be explained by the fact that 23E10-GAL4 expresses in more neurons than 23E10-LexA. In this scenario the absence of VNC-SP neurons in the 23E10-LexA pattern of expression must account for some if not all this difference. However, since 23E10-LexA does not fully recapitulate 652 the expression pattern of 23E10-GAL4 in the dFB, we cannot rule out that some of 653 these missing dFB neurons may be involved in sleep promotion. An alternative explanation is that 23E10-LexA may not be as strong a driver as 23E10-GAL4 and therefore would not express sufficient levels of the CsChrimson construct. In our 656 experience this is very unlikely as we have optogenetically activated other pairs of 657 Gal4/LexA drivers and obtained identical and strong sleep activation with both the LexA 658 and GAL4 counterparts (data not shown). 
$66237,44,45)$ we think that this is very unlikely. Most of the previous work supports a role

663 for dFB neurons in sleep homeostasis. In particular, sleep deprivation increases the

664 excitability of dFB neurons (29) and augmented sleep pressure switches dFB neurons

665 from an electrically silent to an electrically active state (33). Furthermore, reducing the

666 excitability of dFB neurons by reducing levels of the Rho-GTPase-activating protein

667 encoded by the crossveinless-c (cv-c) gene leads to a defect in sleep homeostasis (29).

With this in mind, we wanted to assess whether silencing all 23E10-GAL4 neurons, or

670 just 23E10-GAL4 dFB and VNC-SP neurons separately would affect sleep homeostasis

671 (Figure 8A). As seen in Figure 8B, chronic silencing of all 23E10-GAL4 neurons by

672 expression of Kir2.1 effectively blocks homeostatic sleep rebound when compared with

673 both parental controls. Interestingly, chronic silencing of VNC-SP cells does not disrupt

674 homeostatic sleep rebound (Figure 8C) while silencing of most 23E10-GAL4 dFB

675 neurons (contained in the FBS4 line) does (Figure 8D). These data support a role for

676

23E10-GAL4 dFB neurons in sleep homeostasis, in agreement with previous studies.

677

678 Taken together, our data indicate that the 23E10-GAL4 driver contains at least two

679 groups of sleep-regulating neurons, one located in the dFB that is mostly involved in

680 sleep homeostasis and VNC-SP neurons that are dispensable for sleep homeostasis. 


\section{Discussion}

685 The strength of the fly model lies in its strong genetic techniques, allowing researchers

686 to selectively manipulate discrete populations of neurons and monitor how specific

687 behaviors are affected. To do so, an impressive collection of binary expression systems

688 have been developed. In particular, the GAL4/UAS system (8) has been the keystone of

689 Drosophila neurobiological studies. However, GAL4 drivers are often not specific

690 enough and express in cells outside the region of interest. This can make the task of

691 unequivocally assigning a given behavior to a specific neuron or group of neurons

692 particularly difficult. Importantly, refinement of GAL4 expression pattern can be

693 achieved by employing the intersectional Split-GAL4 technology (11).

694

695 Like mammalian sleep, sleep in Drosophila is regulated by multiple areas in the brain

$696(16,17)$. Particularly, an expanding amount of evidence supports a role for dFB neurons

697 in sleep regulation $(27-30,33,34,37,44,45)$. The most widely used tool to manipulate

698 dFB neurons is the 23E10-GAL4 driver $(33,36)$, and thermogenetic or optogenetic

699 activation of 23E10-GAL4 neurons results in increased sleep (31, 37-39). However,

700 whether 23E10-GAL4 dFB neurons can be considered as a functionally homogeneous

701 group is still unclear. Also unclear is whether the sleep-promotion caused by activation

702 of 23E10-GAL4 neurons is due to dFB neurons. This concern is reinforced by the fact

703 that 23E10-GAL4 expresses in many neurons outside the dFB in the brain and in the

704 VNC (Figure 1, Table 1). In this study, we sought to identify the role of individual 23E10-

705 GAL4 dFB neurons in sleep regulation. 
707 To identify sleep-promoting neurons within the 23E10-GAL4 pattern of expression we adopted a targeted Split-GAL4 strategy combining 22 individual AD lines (selected for the strong dFB expression pattern of their GAL4 counterparts) with a 23E10-DBD line. We screened these 22 novel Split-GAL4 FBS lines behaviorally and anatomically. First, we employed a thermogenetic approach to activate the neurons contained in these 22

712 lines. These experiments identified 9 FBS lines that promote sleep when activated in 713 females and males (Figure 2). An anatomical assessment of each line revealed that 714 most sleep-promoting FBS lines express in some dFB neurons, as expected based on our targeted approach. However, they also express in other areas in the brain and especially in the metathoracic ganglion of the VNC. In fact, we identified two very clear

717 and characteristic processes associated with 4 different VNC neurons in many FBS

718 lines, sleep-promoting or not. The first set of two VNC neurons have typical processes

719 in each leg ganglion and send processes in the brain that terminate in the SEZ. Our 720 data demonstrated that these cells are TPN1 neurons (Figure 4) (54). We observed

721 these neurons in 14 out of the 22 FBS lines. However, we demonstrated that TPN1 neurons are not promoting sleep when activated (Figure 4). The second set of two VNC

723 neurons send processes to the superior medial protocerebrum. Due to their

724 characteristic anatomical features, we named these processes "bowtie". Importantly,

725 these processes are seen in 18 out of 22 FBS lines (and in 8 out of the 9 sleep-

726 promoting lines). The high frequency of observation of these processes in our FBS lines

727 suggest that "bowtie" neurons are commonly expressed in many GAL4 lines. In fact, a

728 MultiColor FlpOut (MCFO) study reported that these neurons are observed in more than

$72960 \%$ of all Janelia GAL4 lines (55). Only one line, FBS4, promotes sleep when 
730 thermogenetically activated and expresses only in dFB cells, containing about $80 \%$ of

731 23E10-GAL4 dFB neurons. FBS4 can thus be considered as a dFB specific driver. We

732 note that the amount of sleep induction triggered by thermogenetic activation of FBS4 is

733 lower than in most other sleep-promoting FBS lines. However, the most surprising

734 finding from our anatomical analysis is FBS5, a line that has no expression in the dFB,

735 and still promotes sleep when activated. This clearly demonstrates that 23E10-GAL4

736 contains sleep-promoting neurons that are not dFB cells.

738 Because temperature has a strong impact on sleep $(42,43,48,49)$, we confirmed our

739 findings using an optogenetic activation method (Figure 3). Most sleep-promoting FBS

740 lines increased sleep when activated using either methods. The notable exception is the

741 dFB specific FBS4 line, which did not affect sleep at all upon optogenetic activation.

742 What is the source of this discrepancy? We can propose several scenarios. The first

743 possible explanation is that the threshold of activation needed for the neurons contained

744 in FBS4 to fire and promote sleep is high. This would imply that thermogenetic

745 activation, which is constant during the $24 \mathrm{~h}$ activation day, provides stronger activation

746 than the fragmented optogenetic protocol. Considering that dFB neurons are subject to

747 dopaminergic regulation (33), we hypothesize that under baseline conditions, dFB

748 neurons are under severe dopaminergic inhibition and that our optogenetic activation

749 protocol is not sufficient to bring these cells above firing threshold. A second possibility

750 is that FBS4 expresses in neurons underlying the effect of high temperature on sleep.

751 This hypothesis is supported by a recent study demonstrating that some of the effects of

752 high temperature on sleep are mediated by GABAergic transmission on dFB neurons 
753 (49). It is thus conceivable, that activating FBS4 neurons thermogenetically may

754 counteract the effect of increasing temperature on sleep. This would explain why we

755 only see a sleep-promoting effect when activating FBS4 using thermogenetic and not

756 optogenetic.

757

758 A further screen demonstrated that the VNC 'bowtie" neurons are sleep-promoting

759 (Figure 4). Activating these cells (renamed VNC-SP neurons) increases sleep, sleep

760 consolidation and sleep depth by increasing arousal thresholds. Moreover, chronic, and

761 acute silencing of VNC-SP neurons reduce sleep and sleep consolidation (Figure 5)

762 confirming that VNC-SP neurons positively regulate sleep. We then sought to discover

763 the neurochemical identity of VNC-SP neurons and identified acetylcholine as a key

764 modulator of sleep in VNC-SP neurons (Figure 6). Reducing cholinergic transmission in

765 VNC-SP neurons reduces sleep and sleep consolidation, an effect mirroring the

766 silencing experiments described earlier. Moreover, we demonstrate that VNC-SP

767 neurons use cholinergic transmission to promote sleep when optogenetically activated.

768

769 Since we observed 'bowtie" processes in most FBS lines, we assume that most of these

770 lines express in VNC-SP neurons. One could therefore wonder why we do not observe

771 total sleep changes when activating these lines? Importantly, our analysis of sleep

772 parameters including day and night sleep bout duration reveals that sleep consolidation

773 is significantly increased using at least one activation protocol with 7 of these "non

774 sleep-promoting" "bowtie" containing lines (FBS28, FBS33, FBS35, FBS57, FBS60,

775 FBS64 and FBS87). Thus, out of the 18 FBS lines that express in VNC-SP neurons, 16 
776 of them increase at least one sleep parameter when activated. The only lines that

777 express in VNC-SP neurons and do not affect any sleep parameters when activated are

778 FBS1 and FBS58. Interestingly, we observed that the GFP signal in the VNC-SP

779 "bowtie" processes of these two lines is weak (Figure 2-figure supplement 1), indicating reduced GAL4 expression in these cells. This observation may provide an explanation

781 for the lack of sleep changes when activating these lines. We also suspect that levels of

782 GAL4 expression in VNC-SP neurons could explain why some FBS lines are promoting 783 sleep more strikingly than others.

Where in the nervous system are these VNC-SP cholinergic signals received? We show 786 that VNC-SP neurons receive synaptic inputs in the VNC and have presynaptic sites in 787 the brain (Figure 6-figure supplement 2). This suggests that VNC-SP neurons are well 788 positioned to receive and integrate sleep-relevant sensory inputs from the periphery in 789 the VNC and that they are sending this information to the brain. The nature of these 790 inputs will require additional investigations that will further uncover the function and role 791 of VNC-SP neurons in sleep regulation. Known modulators of sleep include the immune 792 system and metabolic functions. Based on their location, it is possible that VNC-SP 793 neurons integrate signals relevant to these two sleep-modulating systems.

795 Our data raised important questions about the role of dFB neurons in sleep regulation.

796 Importantly, we show that 23E10-GAL4 dFB neurons are involved in sleep homeostasis 797 as silencing them completely abrogates sleep rebound following a night of sleep 798 deprivation (Figure 8). Our data is in agreement with previous work supporting a role for 
799 dFB neurons in sleep homeostasis $(29,33)$. Interestingly, we also demonstrate that

800 VNC-SP neurons are not part of the homeostatic sleep circuit. These findings suggest

801 that dFB neurons and VNC-SP neurons regulate different aspects and functions of

802 sleep. Here again, further work will determine the role of VNC-SP neurons.

803

804 Finally, our data clearly highlight the need to obtain tools that are as specific as possible when attempting to link a given behavior with a specific neuron or group of neurons. In

806 particular, the frequency at which VNC-SP neurons are observed in existing GAL4 lines

807 (in up to $60 \%$ of all Janelia GAL4 lines) (55) raises some important methodological

808 questions. For example, are VNC-SP neurons present in the expression pattern of a

809 GAL4 line that is known to modulate sleep and is seen as a tool specific for a given

810 structure in the brain? Future studies using GAL4 lines in sleep studies will need to take

811 account of the potential presence of VNC-SP neurons in their expression pattern.

812 Perhaps more importantly, existing data may need to be reinterpreted in the light of our

813 finding.

815 Conclusions

816

817 Our work identified a novel group of cholinergic sleep-promoting neurons located in the

818 VNC in Drosophila. We show that these cells are perfectly positioned to receive and

819 integrate sensory inputs from the periphery and send this information to the brain. We

820 demonstrate that these cells regulate sleep quantity and sleep quality. We further

821 demonstrate that they are not involved in sleep homeostasis whereas 23E10-GAL4 dFB 
822 neurons are. Further work will need to determine the nature of the sleep-relevant

823 information that is relayed by VNC-SP neurons.

824

825 Materials and Methods

826

827 Key Resources Table

828

\begin{tabular}{|c|c|c|c|c|}
\hline $\begin{array}{l}\text { Reagent type } \\
\text { (species) or } \\
\text { resource }\end{array}$ & Designation & $\begin{array}{l}\text { Source or } \\
\text { reference }\end{array}$ & Identifiers & $\begin{array}{l}\text { Additional } \\
\text { information }\end{array}$ \\
\hline $\begin{array}{l}\text { Genetic reagent } \\
(D . \\
\text { melanogaster })\end{array}$ & P\{GMR23E10-GAL4\}attP2 & $\begin{array}{l}\text { Bloomington } \\
\text { Drosophila } \\
\text { Stock Center }\end{array}$ & RRID:BDSC_49032 & \\
\hline $\begin{array}{l}\text { Genetic reagent } \\
(D . \\
\text { melanogaster })\end{array}$ & $\begin{array}{l}\mathrm{w}\left[{ }^{*}\right] ; \mathrm{P}\{\mathrm{y}[+\mathrm{t} 7.7] \mathrm{w}[+\mathrm{mC}]=\mathrm{UAS}- \\
\text { TrpA1(B).K\}attP16 }\end{array}$ & $\begin{array}{l}\text { Bloomington } \\
\text { Drosophila } \\
\text { Stock Center }\end{array}$ & RRID:BDSC_26263 & \\
\hline $\begin{array}{l}\text { Genetic reagent } \\
(D . \\
\text { melanogaster })\end{array}$ & Canton-S & $\begin{array}{l}\text { Laboratory of } \\
\text { Paul Shaw }\end{array}$ & & \\
\hline $\begin{array}{l}\text { Genetic reagent } \\
(D . \\
\text { melanogaster })\end{array}$ & $\begin{array}{l}\mathrm{w}\left[^{\star}\right] ; \mathrm{P}\{\mathrm{y}[+\mathrm{t} 7.7] \\
\mathrm{w}[+\mathrm{mC}]=10 X U A S-I V S- \\
\mathrm{mCD} 8:: G F P\} \text { attP40 }\end{array}$ & $\begin{array}{l}\text { Bloomington } \\
\text { Drosophila } \\
\text { Stock Center }\end{array}$ & RRID:BDSC_32186 & \\
\hline $\begin{array}{l}\text { Genetic reagent } \\
(D . \\
\text { melanogaster })\end{array}$ & $\begin{array}{l}\mathrm{w}\left[{ }^{*}\right] ; \mathrm{P}\{\mathrm{y}[+\mathrm{t} 7.7] \\
\mathrm{w}[+\mathrm{mC}]=10 X U A S-I V S- \\
\mathrm{mCD} 8:: G F P\} a t t P 2\end{array}$ & $\begin{array}{l}\text { Bloomington } \\
\text { Drosophila } \\
\text { Stock Center }\end{array}$ & RRID:BDSC_32185 & \\
\hline $\begin{array}{l}\text { Genetic reagent } \\
(D . \\
\text { melanogaster }) \\
\end{array}$ & $\begin{array}{l}\mathrm{w}[1118] ; \mathrm{P}\{\mathrm{y}[+\mathrm{t} 7.7] \\
\mathrm{w}[+\mathrm{mC}]=23 \mathrm{E} 10- \\
\text { GAL4.DBD\}attP2/TM3 }\end{array}$ & $\begin{array}{l}\text { Bloomington } \\
\text { Drosophila } \\
\text { Stock Center }\end{array}$ & RRID:BDSC_69269 & \\
\hline $\begin{array}{l}\text { Genetic reagent } \\
(D . \\
\text { melanogaster })\end{array}$ & $\begin{array}{l}w[1118] ; P\{y[+t 7.7] \\
w[+m C]=p 65 . A D . U w\} a t t P 40\end{array}$ & $\begin{array}{l}\text { Bloomington } \\
\text { Drosophila } \\
\text { Stock Center }\end{array}$ & RRID:BDSC_71210 & Empty-AD \\
\hline $\begin{array}{l}\text { Genetic reagent } \\
(D . \\
\text { melanogaster) }\end{array}$ & $\mathrm{P}\{\mathrm{R} 84 \mathrm{C} 10-\mathrm{p65.AD}\}$ attP40 & $\begin{array}{l}\text { Bloomington } \\
\text { Drosophila } \\
\text { Stock Center }\end{array}$ & RRID:BDSC_70833 & $\begin{array}{l}\text { AD for FBS } \\
4\end{array}$ \\
\hline $\begin{array}{l}\text { Genetic reagent } \\
(D . \\
\text { melanogaster }) \\
\end{array}$ & $\mathrm{P}\{\mathrm{R} 23 \mathrm{E} 12-\mathrm{p} 65 . \mathrm{AD}\}$ attP40 & $\begin{array}{l}\text { Bloomington } \\
\text { Drosophila } \\
\text { Stock Center }\end{array}$ & RRID:BDSC_70602 & $\begin{array}{l}\text { AD for FBS } \\
5\end{array}$ \\
\hline
\end{tabular}




\begin{tabular}{|c|c|c|c|c|}
\hline $\begin{array}{l}\text { Genetic reagent } \\
(D . \\
\text { melanogaster })\end{array}$ & $\begin{array}{l}\text { w[1118]; P\{y[+t7.7] } \\
\text { w[+mC]=VT050238- } \\
\text { p65.AD\}attP40 }\end{array}$ & $\begin{array}{l}\text { Bloomington } \\
\text { Drosophila } \\
\text { Stock Center }\end{array}$ & RRID:BDSC_72962 & $\begin{array}{l}\text { AD for } F B S \\
45\end{array}$ \\
\hline $\begin{array}{l}\text { Genetic reagent } \\
(D . \\
\text { melanogaster) }\end{array}$ & P\{VT023823-p65.AD\}attP40 & $\begin{array}{l}\text { Bloomington } \\
\text { Drosophila } \\
\text { Stock Center }\end{array}$ & RRID:BDSC_86629 & $\begin{array}{l}\text { AD for } F B S \\
68\end{array}$ \\
\hline $\begin{array}{l}\text { Genetic reagent } \\
(D . \\
\text { melanogaster })\end{array}$ & $P\{R 12 D 12-p 65 . A D\} a t t P 40$ & $\begin{array}{l}\text { Bloomington } \\
\text { Drosophila } \\
\text { Stock Center }\end{array}$ & RRID:BDSC_70539 & $\begin{array}{l}\text { AD for } F B S \\
1\end{array}$ \\
\hline $\begin{array}{l}\text { Genetic reagent } \\
(D . \\
\text { melanogaster) }\end{array}$ & $\mathrm{P}\{\mathrm{R} 42 \mathrm{H} 01-\mathrm{p} 65 . \mathrm{AD}\}$ attP40 & $\begin{array}{l}\text { Bloomington } \\
\text { Drosophila } \\
\text { Stock Center }\end{array}$ & RRID:BDSC_70687 & $\begin{array}{l}A D \text { for } F B S \\
6\end{array}$ \\
\hline $\begin{array}{l}\text { Genetic reagent } \\
(D . \\
\text { melanogaster })\end{array}$ & $P\{R 22 A 12-p 65 . A D\} a t t P 40$ & $\begin{array}{l}\text { Bloomington } \\
\text { Drosophila } \\
\text { Stock Center }\end{array}$ & RRID:BDSC_70120 & $\begin{array}{l}A D \text { for } F B S \\
25\end{array}$ \\
\hline $\begin{array}{l}\text { Genetic reagent } \\
(D . \\
\text { melanogaster })\end{array}$ & P\{R54G07-p65.AD\}attP40 & $\begin{array}{l}\text { Bloomington } \\
\text { Drosophila } \\
\text { Stock Center }\end{array}$ & RRID:BDSC_70737 & $\begin{array}{l}A D \text { for } F B S \\
28\end{array}$ \\
\hline $\begin{array}{l}\text { Genetic reagent } \\
(D . \\
\text { melanogaster) }\end{array}$ & $P\{R 49 C 04-p 65 . A D\} a t t P 40$ & $\begin{array}{l}\text { Bloomington } \\
\text { Drosophila } \\
\text { Stock Center }\end{array}$ & RRID:BDSC_71075 & $\begin{array}{l}A D \text { for } F B S \\
33\end{array}$ \\
\hline $\begin{array}{l}\text { Genetic reagent } \\
(D . \\
\text { melanogaster })\end{array}$ & $P\{R 65 C 03-p 65 . A D\} a t t P 40$ & $\begin{array}{l}\text { Bloomington } \\
\text { Drosophila } \\
\text { Stock Center }\end{array}$ & RRID:BDSC_71005 & $\begin{array}{l}A D \text { for } F B S \\
35\end{array}$ \\
\hline $\begin{array}{l}\text { Genetic reagent } \\
(D . \\
\text { melanogaster) }\end{array}$ & P\{VT058545-p65.AD\}attP40 & $\begin{array}{l}\text { Bloomington } \\
\text { Drosophila } \\
\text { Stock Center }\end{array}$ & RRID:BDSC_74090 & $\begin{array}{l}\text { AD for } F B S \\
41\end{array}$ \\
\hline $\begin{array}{l}\text { Genetic reagent } \\
(D . \\
\text { melanogaster })\end{array}$ & $P\{R 12 G 09-p 65 . A D\} a t t P 40$ & $\begin{array}{l}\text { Bloomington } \\
\text { Drosophila } \\
\text { Stock Center }\end{array}$ & RRID:BDSC_68826 & $\begin{array}{l}\text { AD for } F B S \\
42\end{array}$ \\
\hline $\begin{array}{l}\text { Genetic reagent } \\
(D . \\
\text { melanogaster) }\end{array}$ & P\{VT043400-p65.AD\}attP40 & $\begin{array}{l}\text { Bloomington } \\
\text { Drosophila } \\
\text { Stock Center }\end{array}$ & RRID:BDSC_75874 & $\begin{array}{l}A D \text { for } F B S \\
53\end{array}$ \\
\hline $\begin{array}{l}\text { Genetic reagent } \\
(D . \\
\text { melanogaster })\end{array}$ & P\{VT025720-p65.AD\}attP40 & $\begin{array}{l}\text { Bloomington } \\
\text { Drosophila } \\
\text { Stock Center }\end{array}$ & RRID:BDSC_73432 & $\begin{array}{l}A D \text { for } F B S \\
57\end{array}$ \\
\hline $\begin{array}{l}\text { Genetic reagent } \\
(D . \\
\text { melanogaster) }\end{array}$ & P\{VT064566-p65.AD\}attP40 & $\begin{array}{l}\text { Bloomington } \\
\text { Drosophila } \\
\text { Stock Center }\end{array}$ & RRID:BDSC_71451 & $\begin{array}{l}A D \text { for } F B S \\
58\end{array}$ \\
\hline $\begin{array}{l}\text { Genetic reagent } \\
(D . \\
\text { melanogaster) }\end{array}$ & P\{VT023818-p65.AD\}attP40 & $\begin{array}{l}\text { Bloomington } \\
\text { Drosophila } \\
\text { Stock Center }\end{array}$ & RRID:BDSC_73262 & $\begin{array}{l}A D \text { for } F B S \\
60\end{array}$ \\
\hline $\begin{array}{l}\text { Genetic reagent } \\
(D . \\
\text { melanogaster })\end{array}$ & P\{VT027956-p65.AD\}attP40 & $\begin{array}{l}\text { Bloomington } \\
\text { Drosophila } \\
\text { Stock Center }\end{array}$ & RRID:BDSC_73061 & $\begin{array}{l}A D \text { for } F B S \\
64\end{array}$ \\
\hline
\end{tabular}




\begin{tabular}{|c|c|c|c|c|}
\hline $\begin{array}{l}\text { Genetic reagent } \\
(D . \\
\text { melanogaster })\end{array}$ & P\{VT034811-p65.AD\}attP40 & $\begin{array}{l}\text { Bloomington } \\
\text { Drosophila } \\
\text { Stock Center }\end{array}$ & RRID:BDSC_71337 & $\begin{array}{l}\text { AD for } F B S \\
70\end{array}$ \\
\hline $\begin{array}{l}\text { Genetic reagent } \\
(D . \\
\text { melanogaster) }\end{array}$ & P\{VT026953-p65.AD\}attP40 & $\begin{array}{l}\text { Bloomington } \\
\text { Drosophila } \\
\text { Stock Center }\end{array}$ & RRID:BDSC_74413 & $\begin{array}{l}\text { AD for } F B S \\
72\end{array}$ \\
\hline $\begin{array}{l}\text { Genetic reagent } \\
(D . \\
\text { melanogaster }) \\
\end{array}$ & $\mathrm{P}\{\mathrm{R} 74 \mathrm{G} 10-\mathrm{p} 65 . \mathrm{AD}\}$ attP40 & $\begin{array}{l}\text { Bloomington } \\
\text { Drosophila } \\
\text { Stock Center }\end{array}$ & RRID:BDSC_71131 & $\begin{array}{l}\text { AD for } F B S \\
81\end{array}$ \\
\hline $\begin{array}{l}\text { Genetic reagent } \\
(D . \\
\text { melanogaster) }\end{array}$ & P\{VT007754-p65.AD\}attP40 & $\begin{array}{l}\text { Bloomington } \\
\text { Drosophila } \\
\text { Stock Center }\end{array}$ & RRID:BDSC_74138 & $\begin{array}{l}A D \text { for } F B S \\
84\end{array}$ \\
\hline $\begin{array}{l}\text { Genetic reagent } \\
(D . \\
\text { melanogaster })\end{array}$ & P\{VT045793-p65.AD\}attP40 & $\begin{array}{l}\text { Bloomington } \\
\text { Drosophila } \\
\text { Stock Center }\end{array}$ & RRID:BDSC_71441 & $\begin{array}{l}\text { AD for FBS } \\
87\end{array}$ \\
\hline $\begin{array}{l}\text { Genetic reagent } \\
(D . \\
\text { melanogaster })\end{array}$ & $\begin{array}{l}\mathrm{w}[1118] ; \mathrm{P}\{\mathrm{y}[+\mathrm{t} 7.7] \\
\mathrm{w}[+\mathrm{mC}]=20 X U A S-I V S- \\
\text { CsChrimson.mVenus }\} \text { attP40 }\end{array}$ & $\begin{array}{l}\text { Bloomington } \\
\text { Drosophila } \\
\text { Stock Center }\end{array}$ & RRID:BDSC_55135 & \\
\hline $\begin{array}{l}\text { Genetic reagent } \\
(D . \\
\text { melanogaster) }\end{array}$ & P\{VT020742-p65.AD\}attP40 & $\begin{array}{l}\text { Bloomington } \\
\text { Drosophila } \\
\text { Stock Center }\end{array}$ & RRID:BDSC_73460 & \\
\hline $\begin{array}{l}\text { Genetic reagent } \\
(D . \\
\text { melanogaster })\end{array}$ & $P\{R 30 A 08-p 65 . A D\} a t t P 40$ & $\begin{array}{l}\text { Bloomington } \\
\text { Drosophila } \\
\text { Stock Center }\end{array}$ & RRID:BDSC_71007 & \\
\hline $\begin{array}{l}\text { Genetic reagent } \\
(D . \\
\text { melanogaster) }\end{array}$ & P\{VT013602-p65.AD\}attP40 & $\begin{array}{l}\text { Bloomington } \\
\text { Drosophila } \\
\text { Stock Center }\end{array}$ & RRID:BDSC_74209 & \\
\hline $\begin{array}{l}\text { Genetic reagent } \\
(D . \\
\text { melanogaster })\end{array}$ & P\{GMR30A08-lexA\}attP40 & $\begin{array}{l}\text { Bloomington } \\
\text { Drosophila } \\
\text { Stock Center }\end{array}$ & RRID:BDSC_54756 & \\
\hline $\begin{array}{l}\text { Genetic reagent } \\
(D . \\
\text { melanogaster })\end{array}$ & $\begin{array}{l}\mathrm{y}[1] \mathrm{w}\left[{ }^{*}\right] ; \mathrm{P}\{\mathrm{y}[+\mathrm{t} 7.7] \\
\mathrm{w}[+\mathrm{mC}]=10 X U A S-I V S- \\
\mathrm{mCD} 8:: R F P\} \text { attP18 P\{y[+t7.7] } \\
\text { w[+mC]=13XLexAop2- } \\
\text { mCD8::GFP\}attP8 }\end{array}$ & $\begin{array}{l}\text { Bloomington } \\
\text { Drosophila } \\
\text { Stock Center }\end{array}$ & RRID:BDSC_32229 & \\
\hline $\begin{array}{l}\text { Genetic reagent } \\
(D . \\
\text { melanogaster })\end{array}$ & $\begin{array}{l}\mathrm{w}\left[{ }^{*}\right] ; \mathrm{P}\{\mathrm{y}[+\mathrm{t} 7.7] \\
\mathrm{w}[+\mathrm{mC}]=13 \mathrm{XLexAop2-} \\
\text { KZip+.3XHA\}su(Hw)attP5/CyO; } \\
\text { TM6B, Tb[1]/MKRS }\end{array}$ & $\begin{array}{l}\text { Bloomington } \\
\text { Drosophila } \\
\text { Stock Center }\end{array}$ & RRID:BDSC_76253 & \\
\hline $\begin{array}{l}\text { Genetic reagent } \\
(D . \\
\text { melanogaster) }\end{array}$ & $\begin{array}{l}\mathrm{w}\left[{ }^{\star}\right] ; \mathrm{P}\{\mathrm{y}[+\mathrm{t} 7.7] \\
\mathrm{w}[+\mathrm{mC}]=13 \mathrm{XLExAop} 2- \\
\text { KZip+.3XHA\}attP2 }\end{array}$ & $\begin{array}{l}\text { Bloomington } \\
\text { Drosophila } \\
\text { Stock Center }\end{array}$ & RRID:BDSC_76254 & \\
\hline $\begin{array}{l}\text { Genetic reagent } \\
(D . \\
\text { melanogaster }) \\
\end{array}$ & P\{VT020742-GAL4.DBD\}attP2 & $\begin{array}{l}\text { Bloomington } \\
\text { Drosophila } \\
\text { Stock Center }\end{array}$ & RRID:BDSC_72912 & \\
\hline
\end{tabular}




\begin{tabular}{|c|c|c|c|c|}
\hline $\begin{array}{l}\text { Genetic reagent } \\
\text { (D. } \\
\text { melanogaster) }\end{array}$ & $\begin{array}{l}\mathrm{w}[1118] ; \mathrm{P}\{\mathrm{y}[+\mathrm{t} 7.7] \\
\mathrm{w}[+\mathrm{mC}]=\mathrm{GAL} 4 . \mathrm{DBD} . U \mathrm{w}\} a t t P 2\end{array}$ & $\begin{array}{l}\text { Bloomington } \\
\text { Drosophila } \\
\text { Stock Center }\end{array}$ & RRID:BDSC_71209 & Empty-DBD \\
\hline $\begin{array}{l}\text { Genetic reagent } \\
(D . \\
\text { melanogaster) }\end{array}$ & $\begin{array}{l}\left.w{ }^{*}\right] ; P\{w[+m C]=U A S- \\
\text { Hsap\KCNJ2.EGFP }\} 7\end{array}$ & $\begin{array}{l}\text { Bloomington } \\
\text { Drosophila } \\
\text { Stock Center }\end{array}$ & RRID:BDSC_6595 & UAS-Kir2.1 \\
\hline $\begin{array}{l}\text { Genetic reagent } \\
(D . \\
\text { melanogaster) }\end{array}$ & $\begin{array}{l}\text { pJFRC100-20XUAS-TTS- } \\
\text { Shibire-ts1-p10 in VK00005 }\end{array}$ & $\begin{array}{l}\text { Laboratory of } \\
\text { Gerry Rubin }\end{array}$ & (70) & UAS-Shits1 \\
\hline $\begin{array}{l}\text { Genetic reagent } \\
\text { (D. } \\
\text { melanogaster) }\end{array}$ & GMR-GAL4 & $\begin{array}{l}\text { Laboratory of } \\
\text { Jeff Price }\end{array}$ & (71) & \\
\hline $\begin{array}{l}\text { Genetic reagent } \\
\text { (D. } \\
\text { melanogaster) }\end{array}$ & P\{GawB\}elav[C155] & $\begin{array}{l}\text { Bloomington } \\
\text { Drosophila } \\
\text { Stock Center }\end{array}$ & RRID:BDSC_458 & elav-GAL4 \\
\hline $\begin{array}{l}\text { Genetic reagent } \\
(D . \\
\text { melanogaster })\end{array}$ & P\{TRiP.HMC05021\}attP40 & $\begin{array}{l}\text { Bloomington } \\
\text { Drosophila } \\
\text { Stock Center }\end{array}$ & RRID:BDSC_60028 & $\begin{array}{l}\text { Expresses } \\
\text { dsRNA for } \\
\text { RNAi of } \\
\text { ChAT under } \\
\text { UAS control }\end{array}$ \\
\hline $\begin{array}{l}\text { Genetic reagent } \\
(D . \\
\text { melanogaster) }\end{array}$ & $\begin{array}{l}\mathrm{y}[1] \mathrm{v} \text { [1]; P\{y[+t7.7] } \\
\text { v[+t1.8]=UAS- } \\
\text { GFP.VALIUM10\}attP2 }\end{array}$ & $\begin{array}{l}\text { Bloomington } \\
\text { Drosophila } \\
\text { Stock Center }\end{array}$ & RRID:BDSC_35786 & $\begin{array}{l}\text { Control } \\
\text { RNAi line }\end{array}$ \\
\hline $\begin{array}{l}\text { Genetic reagent } \\
(D . \\
\text { melanogaster })\end{array}$ & $\begin{array}{l}\mathrm{w}[1118] ; \mathrm{P}\{\mathrm{w}[+\mathrm{mC}]=U A S-D c r- \\
\text { 2.D }\} 2\end{array}$ & $\begin{array}{l}\text { Bloomington } \\
\text { Drosophila } \\
\text { Stock Center }\end{array}$ & RRID:BDSC_24650 & $\begin{array}{l}\text { UAS-Dicer2 } \\
\text { on } \\
\text { chromosome } \\
2\end{array}$ \\
\hline $\begin{array}{l}\text { Genetic reagent } \\
(D . \\
\text { melanogaster })\end{array}$ & $\begin{array}{l}w[1118] ; P\{w[+m C]=U A S-D c r- \\
\text { 2.D } 110\end{array}$ & $\begin{array}{l}\text { Bloomington } \\
\text { Drosophila } \\
\text { Stock Center }\end{array}$ & RRID:BDSC_24651 & $\begin{array}{l}\text { UAS-Dicer2 } \\
\text { on } \\
\text { chromosome } \\
3\end{array}$ \\
\hline $\begin{array}{l}\text { Genetic reagent } \\
(D . \\
\text { melanogaster) }\end{array}$ & $\begin{array}{l}\mathrm{w}[1118] ; \mathrm{P}\{\mathrm{y}[+\mathrm{t} 7.7] \\
\mathrm{w}[+\mathrm{mC}]=20 X U A S-I V S- \\
\text { CsChrimson.mVenus\}attP2 }\end{array}$ & $\begin{array}{l}\text { Bloomington } \\
\text { Drosophila } \\
\text { Stock Center }\end{array}$ & RRID:BDSC_55136 & \\
\hline $\begin{array}{l}\text { Genetic reagent } \\
(D . \\
\text { melanogaster) }\end{array}$ & $\begin{array}{l}\mathrm{w}[1118] ; \mathrm{P}\{\mathrm{y}[+\mathrm{t} 7.7] \\
\mathrm{w}[+\mathrm{mC}]=20 X U A S-I V S- \\
\text { CsChrimson.mVenus\}attP18 }\end{array}$ & $\begin{array}{l}\text { Bloomington } \\
\text { Drosophila } \\
\text { Stock Center }\end{array}$ & RRID:BDSC_55134 & \\
\hline $\begin{array}{l}\text { Genetic reagent } \\
(D . \\
\text { melanogaster })\end{array}$ & $\begin{array}{l}\text { w[*]; Mi\{Trojan- } \\
\text { lexA:QFAD.0\}ChAT[MI04508- } \\
\text { TlexA:QFAD.0] } \\
\text { CG7715[MI04508- } \\
\text { TlexA:QFAD.0-X]/TM6B, Tb[1] }\end{array}$ & $\begin{array}{l}\text { Bloomington } \\
\text { Drosophila } \\
\text { Stock Center }\end{array}$ & RRID:BDSC_60319 & ChAT-LexA \\
\hline $\begin{array}{l}\text { Genetic reagent } \\
\text { ( } D . \\
\text { melanogaster) }\end{array}$ & P\{GMR23E10-lexA\}attP40 & $\begin{array}{l}\text { Bloomington } \\
\text { Drosophila } \\
\text { Stock Center }\end{array}$ & RRID:BDSC_52693 & \\
\hline
\end{tabular}




\begin{tabular}{|c|c|c|c|c|}
\hline $\begin{array}{l}\text { Genetic reagent } \\
(D . \\
\text { melanogaster })\end{array}$ & $\begin{array}{l}\mathrm{w}[1118] ; \mathrm{P}\{\mathrm{y}[+\mathrm{t} 7.7] \\
\mathrm{w}[+\mathrm{mC}]=13 X L \text { LexAop2-IVS- } \\
\text { CsChrimson.mVenus }\} \text { attP18 }\end{array}$ & $\begin{array}{l}\text { Bloomington } \\
\text { Drosophila } \\
\text { Stock Center }\end{array}$ & RRID:BDSC_55137 & \\
\hline $\begin{array}{l}\text { Genetic reagent } \\
(D . \\
\text { melanogaster })\end{array}$ & $\begin{array}{l}\mathrm{w}[1118] ; \mathrm{P}\{\mathrm{w}[+\mathrm{mC}]=\mathrm{UAS}- \\
\text { syt.eGFP }\}\end{array}$ & $\begin{array}{l}\text { Bloomington } \\
\text { Drosophila } \\
\text { Stock Center }\end{array}$ & RRID:BDSC_62138 & \\
\hline $\begin{array}{l}\text { Genetic reagent } \\
(D . \\
\text { melanogaster })\end{array}$ & $\begin{array}{l}\mathrm{w}\left[{ }^{*}\right] ; \mathrm{P}\{\mathrm{y}[+\mathrm{t} 7.7] \\
\mathrm{w}[+\mathrm{mC}]=5 \mathrm{XUAS}- \\
\text { DenMark::smGdP- } \\
\text { V5\}su(Hw)attP5 }\end{array}$ & $\begin{array}{l}\text { Bloomington } \\
\text { Drosophila } \\
\text { Stock Center }\end{array}$ & RRID:BDSC_6926 & \\
\hline $\begin{array}{l}\text { Genetic reagent } \\
(D . \\
\text { melanogaster })\end{array}$ & $\begin{array}{l}\mathrm{P}\{\mathrm{TRRP} . J F 01877\} \text { attP2 (RNAi } \\
\text { against ChAT) }\end{array}$ & $\begin{array}{l}\text { Bloomington } \\
\text { Drosophila } \\
\text { Stock Center }\end{array}$ & RRID:BDSC_25856 & $\begin{array}{l}\text { Expresses } \\
\text { dsRNA for } \\
\text { RNAi of } \\
\text { ChAT under } \\
\text { UAS control }\end{array}$ \\
\hline Antibody & Anti-GFP (Chicken polyclonal) & Aves Labs, Inc & GFP-1020 & $\begin{array}{l}\text { dilution } \\
1: 1000\end{array}$ \\
\hline Antibody & $\begin{array}{l}\text { anti-bruchpilot (mouse } \\
\text { monoclonal) }\end{array}$ & $\begin{array}{l}\text { Developmental } \\
\text { Studies } \\
\text { Hybridoma } \\
\text { Bank }\end{array}$ & nc82-s & dilution 1:50 \\
\hline Antibody & Anti-DsRed (rabbit polyclonal) & Takara & 632496 & $\begin{array}{l}\text { dilution } \\
1: 250\end{array}$ \\
\hline Antibody & $\begin{array}{l}\text { Anti-ChAT (mouse } \\
\text { monoclonal) }\end{array}$ & $\begin{array}{l}\text { Developmental } \\
\text { Studies } \\
\text { Hybridoma } \\
\text { Bank }\end{array}$ & Chat4B1-c & $\begin{array}{l}\text { dilution } \\
1: 100\end{array}$ \\
\hline Antibody & Anti-VGlut (rabbit) & $\begin{array}{l}\text { Aaron } \\
\text { DiAntonio } \\
\text { (Washington } \\
\text { University) }\end{array}$ & (72) & $\begin{array}{l}\text { dilution } \\
1: 500\end{array}$ \\
\hline Antibody & Anti-GABA (rabbit polyclonal) & Sigma-Aldrich & A2052 & $\begin{array}{l}\text { dilution } \\
1: 1000\end{array}$ \\
\hline Antibody & Anti-5-HT (rabbit polyclonal) & Sigma-Aldrich & S5545 & $\begin{array}{l}\text { dilution } \\
1: 500 \\
\end{array}$ \\
\hline Antibody & Anti-TH (mouse monoclonal) & Immunostar & 22941 & $\begin{array}{l}\text { dilution } \\
1: 100\end{array}$ \\
\hline Antibody & Anti-Tdc2 (rabbit polyclonal) & Covalab & pab0822-P & $\begin{array}{l}\text { dilution } \\
1: 200\end{array}$ \\
\hline Antibody & Anti-V5 (mouse monoclonal) & Sigma-Aldrich & V8012 & $\begin{array}{l}\text { dilution } \\
1: 400\end{array}$ \\
\hline Antibody & $\begin{array}{l}\text { Goat anti-chicken Alexafluor } \\
488\end{array}$ & Invitrogen & A32931 & $\begin{array}{l}\text { dilution } \\
1: 800 \\
\end{array}$ \\
\hline Antibody & $\begin{array}{l}\text { Goat anti-mouse Alexafluor } \\
555\end{array}$ & Invitrogen & A32727 & $\begin{array}{l}\text { dilution } \\
1: 400\end{array}$ \\
\hline
\end{tabular}




\begin{tabular}{|c|c|c|c|c|}
\hline Antibody & Goat anti-rabbit Alexafluor 555 & Invitrogen & A32732 & $\begin{array}{l}\text { dilution } \\
1: 400\end{array}$ \\
\hline Antibody & $\begin{array}{l}\text { Goat anti-mouse Alexafluor } \\
633\end{array}$ & Invitrogen & A21052 & $\begin{array}{l}\text { dilution } \\
1: 400\end{array}$ \\
\hline Antibody & Goat anti-rabbit Alexafluor 633 & Invitrogen & A21071 & $\begin{array}{l}\text { dilution } \\
1: 400\end{array}$ \\
\hline $\begin{array}{l}\text { Chemical } \\
\text { compound, drug }\end{array}$ & retinal & Sigma & R2500 & $400 \mu \mathrm{M}$ \\
\hline $\begin{array}{l}\text { Chemical } \\
\text { compound, drug }\end{array}$ & Vectashield & $\begin{array}{l}\text { Vector } \\
\text { Laboratories }\end{array}$ & $\mathrm{H}-1000$ & \\
\hline $\begin{array}{l}\text { Chemical } \\
\text { compound, drug }\end{array}$ & $20 \%$ paraformaldehyde & $\begin{array}{l}\text { Electron } \\
\text { Microscopy } \\
\text { Sciences }\end{array}$ & 15713 & $4 \%$ dilution \\
\hline Media & $\begin{array}{l}\text { Drosophila Schneider's } \\
\text { Medium }\end{array}$ & Gibco & 21720024 & \\
\hline Media & Normal Goat Serum & $\begin{array}{l}\text { MP } \\
\text { Biomedicals }\end{array}$ & 191356 & $5 \%$ dilution \\
\hline
\end{tabular}

829

$830 \quad$ Flies

831 Flies were cultured at $25^{\circ} \mathrm{C}$ with $50 \%$ humidity under a 12-hour light: 12-hour dark cycle.

832 Flies were kept on a standard yeast and molasses diet (per 1L: $50 \mathrm{~g}$ yeast, $15 \mathrm{~g}$ sucrose,

$83328 \mathrm{~g}$ corn syrup, 33.3mL molasses, $9 \mathrm{~g}$ agar).

Sleep

836 Sleep was assessed as previously described $(32,73) .4-10$ day old virgin females or

837 males were used as described in figure legends. Briefly, flies were placed into individual

$83865 \mathrm{~mm}$ tubes and all activity was continuously measured through the Trikinetics

839 Drosophila Activity Monitoring System (www.Trikinetics.com, Waltham, Ma). Locomotor

840 activity was measured in 1-minute bins and sleep was defined as periods of quiescence

841 lasting at least 5 minutes. 


\section{Optogenetic activation}

843 For optogenetic activation, flies expressing CsChrimson were loaded into individual

$84465 \mathrm{~mm}$ tubes with food supplemented with 400 $\mu \mathrm{M}$ all-trans retinal (Sigma, \#R2500) and

845 kept in the dark for 48 hours. Sleep was then monitored under low-light conditions for 48

846 hours of baseline measurements. To activate CSChrimson, flies were put under $627 \mathrm{~nm}$

847 LEDs (LuxeonStar LXM2-PD01-0040) set to a pulse cycle of (5ms on, 95ms off) x 20

848 with a 4 second delay between pulse cycles. The light intensity of this protocol is

$8490.024 \mathrm{~mW} / \mathrm{mm}^{2}$

850

851 Sleep deprivation

852 Sleep deprivation was performed as previously described $(32,74)$. Briefly, flies were

853 placed into individual $65 \mathrm{~mm}$ tubes and the sleep-nullifying apparatus (SNAP) was used

854 to sleep deprive flies for 12 hours during the dark phase. Sleep homeostasis was

855 calculated for each individual fly as the ratio of the minutes of sleep gained above

856 baseline during $24 \mathrm{~h}$ of recovery sleep divided by the total min of sleep lost during $12 \mathrm{~h}$

857 of sleep deprivation.

858

859 Arousal threshold

860 Retinal or vehicle-fed VNC-SP>UAS-CsChrimson flies were placed into individual 65

$861 \mathrm{~mm}$ tubes in Trikinetics monitors. These were then loaded in the SNAP sleep

862 deprivation apparatus. The SNAP tilts asymmetrically from $-60^{\circ}$ to $+60^{\circ}$, such that

863 sleeping flies are disturbed during each downward movement (75). Each individual 
864 SNAP movement takes 15 seconds to complete. To assess arousal threshold, we

865 modified the SNAP parameters so that flies were subjected to only 1, 2, or 4 downward

866 movements in the SNAP every hour for $24 \mathrm{~h}$ and their locomotor responses were

867 analyzed. We then averaged each individual hour response to obtain the percentage of

868 flies awakened by a given strength of perturbation.

870 QPCR

871 QPCR were performed as previously described $(32,76)$. Briefly, total RNA was isolated

872 from the bodies and eyes of $\sim 10$ flies per replicate with Trizol (Invitrogen, Carlsbad, CA)

873 and DNAse I digested. cDNA synthesis was performed using Superscript IV VILO

874 (Invitrogen) according to manufacturer protocol. cDNA samples were loaded in

875 duplicate and quantitatively analyzed using SYBR Green Master Mix (Applied

876 Biosystems) and gene specific primers using the Applied Biosystems 7500 system.

877 Expression values for RP49 were used to normalize results between groups. The

878 following primer sets were used: rp49: fw aagaagcgcaccaagcacttcatc, rev

879 tctgttgtcgatacccttgggctt and ChAT: fw caccgagcgatacaggatgg, rev

880 ggcaccttgggtagagtgtc.

883 Flies were cold anesthetized, brains and VNC were dissected in ice cold Schneider's

884 Drosophila Medium (Gibco, 21720024) and blocked in 5\% normal goat serum.

885 Following blocking flies were incubated overnight at $4^{\circ} \mathrm{C}$ in primary antibody, washed in 
886 PBST and incubated overnight at $4^{\circ} \mathrm{C}$ in secondary antibody. Description of antibodies

887 used can be found in the resources table. Brains and VNCs were mounted on polylysine

888 treated slides in Vectashield $\mathrm{H}-1000$ mounting medium. Imaging was performed on a

889 Zeiss 510 meta confocal microscope using a Plan-Apochromat 20x or Plan-Neofluar

$89040 x$ objective. Z-series images were acquired with a 1 uM slice size using the same

891 settings (laser power, gain, offset) to allow for comparison across genotypes. Images

892 were processed and analyzed using ImageJ.

894 Statistics

895 Statistical analyses were performed with Prism9 software (GraphPad). Normal

896 distribution was assessed with the D'Agostino-Pearson test. Normally distributed data

897 were analyzed with parametric statistics: t-test, one-way analysis of variance or two-way

898 ANOVA followed by the planned pairwise multiple comparisons as described in the

899 legends. For data that significantly differed from the normal distribution, non-parametric

900 statistics were applied, Mann-Whitney U test or Kruskal-Wallis test followed by Dunn's

901 multiple test. Some non-normally distributed data were subjected to log transformation

902 or Box-Cox transformation before two-way ANOVA followed by planned pairwise

903 multiple comparisons as described in the legends. All statistically different groups are

904 defined as ${ }^{*} \mathrm{P}<0.05,{ }^{* *} \mathrm{P}<0.01,{ }^{* *} \mathrm{P}<0.001$ and ${ }^{* * * *} \mathrm{P}<0.0001$

905 
S.D., J.J. and J.M.E. designed the experiments and wrote the paper. J.J., B.H., K.E.,

A.V., A.V., A.E., J.M.E and S.D. performed the experiments. J.J., J.M.E and S.D.

analyzed the data.

\section{Acknowledgments}

We thank Aaron DiAntonio, Gerry Rubin, Paul Shaw and Jeff Price for sharing reagents.

913 We also thank Krishna Melnattur for comments on this manuscript.

915 Competing Interests

916 The authors have no competing interests.

\section{References}

919

920

921

922

923

924

925

926

927

928

929

930

931

932

933

934

935

936

937

938

1. Park HG, Carmel JB. Selective Manipulation of Neural Circuits. Neurotherapeutics. 2016;13(2):311-24.

2. Moulin TC, Covill LE, Itskov PM, Williams MJ, Schioth HB. Rodent and fly models in behavioral neuroscience: An evaluation of methodological advances, comparative research, and future perspectives. Neurosci Biobehav Rev. 2021;120:1-12.

3. Yamamoto D, Koganezawa M. Genes and circuits of courtship behaviour in Drosophila males. Nat Rev Neurosci. 2013;14(10):681-92.

4. Anderson DJ. Circuit modules linking internal states and social behaviour in flies and mice. Nat Rev Neurosci. 2016;17(11):692-704.

5. Bhumika, Singh AK. Regulation of feeding behavior in Drosophila through the interplay of gustation, physiology and neuromodulation. Front Biosci (Landmark Ed). 2018;23:2016-27.

6. Scott K. Gustatory Processing in Drosophila melanogaster. Annu Rev Entomol. 2018;63:15-30.

7. Ly S, Pack Al, Naidoo N. The neurobiological basis of sleep: Insights from Drosophila. Neurosci Biobehav Rev. 2018;87:67-86.

8. Brand $\mathrm{AH}$, Perrimon N. Targeted gene expression as a means of altering cell fates and generating dominant phenotypes. Development. 1993;118(2):401-15.

9. Lai SL, Lee T. Genetic mosaic with dual binary transcriptional systems in Drosophila. Nat Neurosci. 2006;9(5):703-9.

10. Potter CJ, Tasic B, Russler EV, Liang L, Luo L. The Q system: a repressible binary system for transgene expression, lineage tracing, and mosaic analysis. Cell. 2010;141(3):536-48. 
11. Luan H, Peabody NC, Vinson CR, White BH. Refined spatial manipulation of neuronal function by combinatorial restriction of transgene expression. Neuron. 2006;52(3):425-36.

12. Keene AC, Duboue ER. The origins and evolution of sleep. J Exp Biol. 2018;221(Pt 11).

13. Borbely AA. A two process model of sleep regulation. Hum Neurobiol. 1982;1(3):195-204.

14. Krause AJ, Simon EB, Mander BA, Greer SM, Saletin JM, Goldstein-Piekarski AN, et al. The sleepdeprived human brain. Nat Rev Neurosci. 2017;18(7):404-18.

15. Miyazaki S, Liu CY, Hayashi Y. Sleep in vertebrate and invertebrate animals, and insights into the function and evolution of sleep. Neurosci Res. 2017;118:3-12.

16. Shafer OT, Keene AC. The Regulation of Drosophila Sleep. Curr Biol. 2021;31(1):R38-R49.

17. Dissel S. Drosophila as a Model to Study the Relationship Between Sleep, Plasticity, and Memory. Front Physiol. 2020;11:533.

18. Strauss R. The central complex and the genetic dissection of locomotor behaviour. Curr Opin Neurobiol. 2002;12(6):633-8.

19. Sakai T, Kitamoto T. Differential roles of two major brain structures, mushroom bodies and central complex, for Drosophila male courtship behavior. J Neurobiol. 2006;66(8):821-34.

20. Joiner MA, Griffith LC. Mapping of the anatomical circuit of CaM kinase-dependent courtship conditioning in Drosophila. Learn Mem. 1999;6(2):177-92.

21. Sakai T, Inami S, Sato S, Kitamoto T. Fan-shaped body neurons are involved in period-dependent regulation of long-term courtship memory in Drosophila. Learn Mem. 2012;19(12):571-4.

22. Hu W, Peng Y, Sun J, Zhang F, Zhang X, Wang L, et al. Fan-Shaped Body Neurons in the

Drosophila Brain Regulate Both Innate and Conditioned Nociceptive Avoidance. Cell Rep. 2018;24(6):1573-84.

23. Liu G, Seiler H, Wen A, Zars T, Ito $K$, Wolf R, et al. Distinct memory traces for two visual features in the Drosophila brain. Nature. 2006;439(7076):551-6.

24. Weir PT, Dickinson MH. Functional divisions for visual processing in the central brain of flying Drosophila. Proc Natl Acad Sci U S A. 2015;112(40):E5523-32.

25. Kacsoh BZ, Bozler J, Hodge S, Bosco G. Neural circuitry of social learning in Drosophila requires multiple inputs to facilitate inter-species communication. Commun Biol. 2019;2:309.

26. Sareen PF, McCurdy LY, Nitabach MN. A neuronal ensemble encoding adaptive choice during sensory conflict in Drosophila. Nat Commun. 2021;12(1):4131.

27. Donlea JM, Thimgan MS, Suzuki Y, Gottschalk L, Shaw PJ. Inducing sleep by remote control facilitates memory consolidation in Drosophila. Science. 2011;332(6037):1571-6.

28. Ueno T, Tomita J, Tanimoto H, Endo K, Ito K, Kume S, et al. Identification of a dopamine pathway that regulates sleep and arousal in Drosophila. Nat Neurosci. 2012;15(11):1516-23.

29. Donlea JM, Pimentel D, Miesenbock G. Neuronal Machinery of Sleep Homeostasis in Drosophila. Neuron. 2014;81(6):1442.

30. Liu Q, Liu S, Kodama L, Driscoll MR, Wu MN. Two dopaminergic neurons signal to the dorsal fanshaped body to promote wakefulness in Drosophila. Curr Biol. 2012;22(22):2114-23.

31. Dag U, Lei Z, Le JQ, Wong A, Bushey D, Keleman K. Neuronal reactivation during post-learning sleep consolidates long-term memory in Drosophila. Elife. 2019;8.

32. Dissel S, Angadi V, Kirszenblat L, Suzuki Y, Donlea J, Klose M, et al. Sleep restores behavioral plasticity to Drosophila mutants. Curr Biol. 2015;25(10):1270-81.

33. Pimentel D, Donlea JM, Talbot CB, Song SM, Thurston AJF, Miesenbock G. Operation of a homeostatic sleep switch. Nature. 2016;536(7616):333-7.

34. Kempf A, Song SM, Talbot CB, Miesenbock G. A potassium channel beta-subunit couples mitochondrial electron transport to sleep. Nature. 2019;568(7751):230-4.

35. Alam MA, Kumar S, McGinty D, Alam MN, Szymusiak R. Neuronal activity in the preoptic hypothalamus during sleep deprivation and recovery sleep. J Neurophysiol. 2014;111(2):287-99. 
36. Jenett A, Rubin GM, Ngo TT, Shepherd D, Murphy C, Dionne H, et al. A GAL4-driver line resource for Drosophila neurobiology. Cell Rep. 2012;2(4):991-1001.

37. Qian Y, Cao Y, Deng B, Yang G, Li J, Xu R, et al. Sleep homeostasis regulated by 5HT2b receptor in a small subset of neurons in the dorsal fan-shaped body of drosophila. Elife. 2017;6.

38. Yap MHW, Grabowska MJ, Rohrscheib C, Jeans R, Troup M, Paulk AC, et al. Oscillatory brain activity in spontaneous and induced sleep stages in flies. Nat Commun. 2017;8(1):1815.

39. Berry JA, Cervantes-Sandoval I, Chakraborty M, Davis RL. Sleep Facilitates Memory by Blocking Dopamine Neuron-Mediated Forgetting. Cell. 2015;161(7):1656-67.

40. Hulse BK, Haberkern H, Franconville R, Turner-Evans DB, Takemura SY, Wolff T, et al. A connectome of the Drosophila central complex reveals network motifs suitable for flexible navigation and context-dependent action selection. Elife. 2021;10.

41. Hamada FN, Rosenzweig M, Kang K, Pulver SR, Ghezzi A, Jegla TJ, et al. An internal thermal sensor controlling temperature preference in Drosophila. Nature. 2008;454(7201):217-20.

42. Ishimoto H, Lark A, Kitamoto T. Factors that Differentially Affect Daytime and Nighttime Sleep in Drosophila melanogaster. Front Neurol. 2012;3:24.

43. Lamaze A, Ozturk-Colak A, Fischer R, Peschel N, Koh K, Jepson JE. Regulation of sleep plasticity by a thermo-sensitive circuit in Drosophila. Sci Rep. 2017;7:40304.

44. Donlea JM, Pimentel D, Talbot CB, Kempf A, Omoto JJ, Hartenstein V, et al. Recurrent Circuitry for Balancing Sleep Need and Sleep. Neuron. 2018;97(2):378-89 e4.

45. Tainton-Heap LAL, Kirszenblat LC, Notaras ET, Grabowska MJ, Jeans R, Feng K, et al. A Paradoxical Kind of Sleep in Drosophila melanogaster. Curr Biol. 2021;31(3):578-90 e6.

46. Tirian L, Dickson BJ. The VT GAL4, LexA, and split-GAL4 driver line collections for targeted expression in the Drosophila nervous system. BioRxiv 198648 [Preprint] October 5, 2017 Available from: https://doiorg/101101/198648. 2017.

47. Dionne H, Hibbard KL, Cavallaro A, Kao JC, Rubin GM. Genetic Reagents for Making Split-GAL4 Lines in Drosophila. Genetics. 2018;209(1):31-5.

48. Parisky KM, Agosto Rivera JL, Donelson NC, Kotecha S, Griffith LC. Reorganization of Sleep by Temperature in Drosophila Requires Light, the Homeostat, and the Circadian Clock. Curr Biol. 2016;26(7):882-92.

49. Kim JH, Ki Y, Lee H, Hur MS, Baik B, Hur JH, et al. The voltage-gated potassium channel Shaker promotes sleep via thermosensitive GABA transmission. Commun Biol. 2020;3(1):174.

50. van Alphen B, Yap MH, Kirszenblat L, Kottler B, van Swinderen B. A dynamic deep sleep stage in Drosophila. J Neurosci. 2013;33(16):6917-27.

51. Isaac RE, Li C, Leedale AE, Shirras AD. Drosophila male sex peptide inhibits siesta sleep and promotes locomotor activity in the post-mated female. Proc Biol Sci. 2010;277(1678):65-70.

52. Khericha M, Kolenchery JB, Tauber E. Neural and non-neural contributions to sexual dimorphism of mid-day sleep in Drosophila melanogaster: a pilot study. Physiol Entomol. 2016;41(4):327-34.

53. Wu B, Ma L, Zhang E, Du J, Liu S, Price J, et al. Sexual dimorphism of sleep regulated by juvenile hormone signaling in Drosophila. PLoS Genet. 2018;14(4):e1007318.

54. Kim H, Kirkhart C, Scott K. Long-range projection neurons in the taste circuit of Drosophila. Elife. 2017;6.

55. Meissner GW, Dorman Z, Nern A, Forster K, Gibney T, Jeter J, et al. An image resource of subdivided <em>Drosophila</em> GAL4-driver expression patterns for neuron-level searches. bioRxiv. 2020:2020.05.29.080473.

56. Klapoetke NC, Murata Y, Kim SS, Pulver SR, Birdsey-Benson A, Cho YK, et al. Independent optical excitation of distinct neural populations. Nat Methods. 2014;11(3):338-46. 
57. Otsuna H, Ito M, Kawase T. Color depth MIP mask search: a new tool to expedite Split-GAL4 creation. BioRxiv 318006 [Preprint] May 9, 2018 Available from: https://doiorg/101101/318006v1. 2018.

58. Dolan MJ, Luan H, Shropshire WC, Sutcliffe B, Cocanougher B, Scott RL, et al. Facilitating NeuronSpecific Genetic Manipulations in Drosophila melanogaster Using a Split GAL4 Repressor. Genetics. 2017;206(2):775-84.

59. Baines RA, Uhler JP, Thompson A, Sweeney ST, Bate M. Altered electrical properties in Drosophila neurons developing without synaptic transmission. J Neurosci. 2001;21(5):1523-31. 60. Kitamoto T. Conditional modification of behavior in Drosophila by targeted expression of a temperature-sensitive shibire allele in defined neurons. J Neurobiol. 2001;47(2):81-92.

61. Lacin H, Chen HM, Long X, Singer RH, Lee T, Truman JW. Neurotransmitter identity is acquired in a lineage-restricted manner in the Drosophila CNS. Elife. 2019;8.

62. Diao F, Ironfield H, Luan H, Diao F, Shropshire WC, Ewer J, et al. Plug-and-play genetic access to drosophila cell types using exchangeable exon cassettes. Cell Rep. 2015;10(8):1410-21.

63. Ni JD, Gurav AS, Liu W, Ogunmowo TH, Hackbart H, Elsheikh A, et al. Differential regulation of the Drosophila sleep homeostat by circadian and arousal inputs. Elife. 2019;8.

64. Allen AM, Neville MC, Birtles S, Croset V, Treiber CD, Waddell S, et al. A single-cell transcriptomic atlas of the adult Drosophila ventral nerve cord. Elife. 2020;9.

65. Court R, Namiki S, Armstrong JD, Borner J, Card G, Costa M, et al. A Systematic Nomenclature for the Drosophila Ventral Nerve Cord. Neuron. 2020;107(6):1071-9 e2.

66. Tsubouchi A, Yano T, Yokoyama TK, Murtin C, Otsuna H, Ito K. Topological and modality-specific representation of somatosensory information in the fly brain. Science. 2017;358(6363):615-23.

67. Nicolai L, Ramaekers A, Raemaekers T, Drozdzecki A, Mauss AS, Yan J, et al. Genetically encoded dendritic marker sheds light on neuronal connectivity in Drosophila. Proc Natl Acad Sci U S A. 2010;107(47):20553-8.

68. Zhang YQ, Rodesch CK, Broadie K. Living synaptic vesicle marker: synaptotagmin-GFP. Genesis. 2002;34(1-2):142-5.

69. Pfeiffer BD, Ngo TT, Hibbard KL, Murphy C, Jenett A, Truman JW, et al. Refinement of tools for targeted gene expression in Drosophila. Genetics. 2010;186(2):735-55.

70. Pfeiffer BD, Truman JW, Rubin GM. Using translational enhancers to increase transgene expression in Drosophila. Proc Natl Acad Sci U S A. 2012;109(17):6626-31.

71. Takahashi Y, Hirose F, Matsukage A, Yamaguchi M. Identification of three conserved regions in the DREF transcription factors from Drosophila melanogaster and Drosophila virilis. Nucleic Acids Res. 1999;27(2):510-6.

72. Daniels RW, Collins CA, Chen K, Gelfand MV, Featherstone DE, DiAntonio A. A single vesicular glutamate transporter is sufficient to fill a synaptic vesicle. Neuron. 2006;49(1):11-6.

73. Shaw PJ, Cirelli C, Greenspan RJ, Tononi G. Correlates of sleep and waking in Drosophila melanogaster. Science. 2000;287(5459):1834-7.

74. Seugnet L, Suzuki Y, Vine L, Gottschalk L, Shaw PJ. D1 receptor activation in the mushroom bodies rescues sleep-loss-induced learning impairments in Drosophila. Curr Biol. 2008;18(15):1110-7. 75. Shaw PJ, Tononi G, Greenspan RJ, Robinson DF. Stress response genes protect against lethal effects of sleep deprivation in Drosophila. Nature. 2002;417(6886):287-91.

76. Dissel S, Seugnet L, Thimgan MS, Silverman N, Angadi V, Thacher PV, et al. Differential activation of immune factors in neurons and glia contribute to individual differences in resilience/vulnerability to sleep disruption. Brain Behav Immun. 2015;47:75-85. 


\section{Figure Legends}

Figure 1. The 23E10-GAL4 driver contains many neurons outside the dFB.

1081

1082

1083

1084

1085

1086

1087

1088

1089

1090

1091

1092

1093

1094

1095

1096

1097

1098

1099

1100

1101

1102

1103

1104

1105

1106

A) Diagram of the experimental assay. Sleep was measured at $22^{\circ} \mathrm{C}$ for 2 days to establish baseline sleep profile. Flies were then shifted to $31^{\circ} \mathrm{C}$ for $24 \mathrm{~h}$ at the start of day3 to increase activity of the targeted cells by activating the TrpA1 channel, and then returned to $22^{\circ} \mathrm{C}$. White bars $(\mathrm{L})$ represent the $12 \mathrm{~h}$ of light and black bars $(\mathrm{D})$ represent the $12 \mathrm{~h}$ of dark that are oscillating daily.

B-C) Sleep profile in minutes of sleep per hour for day $2\left(22^{\circ} \mathrm{C}\right.$, blue line $)$ and day 3 $\left(31^{\circ} \mathrm{C}\right.$, red line) for parental control female flies: $23 E 10-G A L 4 /+(B)$ and UAS-TrpA1/+ (C).

D) Sleep profile in minutes of sleep per hour for day $2\left(22^{\circ} \mathrm{C}\right.$, blue line $)$ and day $3\left(31^{\circ} \mathrm{C}\right.$, red line) for 23E10-GAL4>UAS-TrpA1 female flies.

E) Box plots of total sleep change in \% ((total sleep on day3-total sleep on day2/total sleep on day2) X 100) for data presented in B-D. The bottom and top of each box represents the first and third quartile, and the horizontal line dividing the box is the median. The whiskers represent the $10^{\text {th }}$ and $90^{\text {th }}$ percentiles. Flies expressing UASTrpA1 in 23E10-GAL4 significantly increase sleep when switched to $31^{\circ} \mathrm{C}$ compared with parental controls, Kruskal-Wallis ANOVA followed by Dunn's multiple comparisons. ${ }^{* * * *} \mathrm{P}<0.0001, \mathrm{n}=25-31$ flies per genotype.

F) Box plots of locomotor activity counts per minute awake for flies presented in B-D. The bottom and top of each box represents the first and third quartile, and the horizontal line dividing the box is the median. The whiskers represent the $10^{\text {th }}$ and $90^{\text {th }}$ percentiles. Two-way repeated measures ANOVA followed by Sidak's multiple comparisons test found no differences in locomotor activity between $22^{\circ} \mathrm{C}$ and $31^{\circ} \mathrm{C}$. n.s. $=$ not significant. $\mathrm{n}=25-31$ flies per genotype.

G-H) Representative confocal stacks of a female 23E10-GAL4>UAS-mCD8GFP brain (G) and ventral nerve cord (VNC, H). Green, anti-GFP; magenta, anti-nc82 (neuropile marker). 


\section{Figure 2: Thermogenetic screen of FBS lines.}

1108 A) Diagram of the experimental assay. Sleep was measured at $22^{\circ} \mathrm{C}$ for 2 days to 1109 establish baseline sleep profile. Flies were then shifted to $31^{\circ} \mathrm{C}$ for $24 \mathrm{~h}$ at the start of 1110 day3 to increase activity of the targeted cells by activating the TrpA1 channel, and then 1111 returned to $22^{\circ} \mathrm{C}$. White bars $(\mathrm{L})$ represent the $12 \mathrm{~h}$ of light and black bars (D) represent 1112 the $12 \mathrm{~h}$ of dark that are oscillating daily.

1113 B) Box plots of total sleep change in \% ((total sleep on day3-total sleep on day2/total 1114 sleep on day2) X 100) for female control (Empty-AD; 23E10-DBD) and 22 FBS lines 1115 expressing UAS-TrpA1; UAS-GFP. The bottom and top of each box represents the first and third quartile, and the horizontal line dividing the box is the median. The whiskers

1117 represent the $10^{\text {th }}$ and $90^{\text {th }}$ percentiles. The gray rectangle spanning the horizontal axis 1118 indicates the interquartile range of the control. Kruskal-Wallis ANOVA followed by

1119 Dunn's multiple comparisons revealed that 9 FBS lines increase sleep significantly more 1120 than control flies when transferred to $31^{\circ} \mathrm{C}$. ${ }^{* *} \mathrm{P}<0.01,{ }^{* *} \mathrm{P}<0.001,{ }^{* * * *} \mathrm{P}<0.0001, \mathrm{n}=30$ 112151 flies per genotype.

1122 C) Box plots of locomotor activity counts per minute awake for flies presented in $B$. The 1123 bottom and top of each box represents the first and third quartile, and the horizontal line 1124 dividing the box is the median. The whiskers represent the $10^{\text {th }}$ and $90^{\text {th }}$ percentiles.

1125 Two-way repeated measures ANOVA followed by Sidak's multiple comparisons test 1126 found that for 3 sleep-promoting FBS lines (FBS5, FBS45 and FBS53) locomotor 1127 activity per awake time is increased while no differences are seen for the other 6 sleep1128 promoting lines between $22^{\circ} \mathrm{C}$ and $31^{\circ} \mathrm{C}$. ${ }^{* *} \mathrm{P}<0.01,{ }^{* *} \mathrm{P}<0.001,{ }^{* * *} \mathrm{P}<0.0001, \mathrm{n}=30-51$

1129 flies per genotype.

1130 D-H) Left, sleep profile in minutes of sleep per hour for day $2\left(22^{\circ} \mathrm{C}\right.$, blue line $)$ and day 3 $1131\left(31^{\circ} \mathrm{C}\right.$, red line) for empty control (Empty-AD; 23E10-DBD, D), FBS4 (E), FBS5 (F), 1132 FBS45 $(\mathrm{G})$ and FBS68 $(\mathrm{H})$ female flies expressing TrpA1 and GFP. Right,

1133 Representative confocal stacks for each FBS>UAS-TrpA1; UAS-mCD8GFP line of a 1134 female brain (left panel), VNC (middle panel) as well as a magnified view of VNC 1135 "bowtie" processes as indicated by the gray rectangle in F. Yellow arrows in F indicate 1136 TPN1-like processes in FBS5. Green, anti-GFP; magenta, anti-nc82 (neuropile marker). 
1137 Figure 3: Optogenetic screen of FBS lines.

1138 A) Diagram of the experimental assay. Sleep was measured in retinal-fed and vehicle-

1139 fed flies for 2 days without 627nm LED stimulation to establish baseline sleep profile.

1140 LEDs were were then turned on for $24 \mathrm{~h}$ at the start of day3 to increase activity of the

1141 targeted cells by activating the CsChrimson channel, and then turned off on day4. White

1142 bars $(\mathrm{L})$ represent the $12 \mathrm{~h}$ of light and black bars (D) represent the $12 \mathrm{~h}$ of dark that are

1143 oscillating daily.

1144 B) Box plots of total sleep change in \% ((total sleep on day3-total sleep on day2/total 1145 sleep on day2) X 100) for control (Empty) and 22 experimental vehicle-fed and retinal-

1146 fed female flies expressing CsChrimson upon 627nm LED stimulation. The bottom and

1147 top of each box represents the first and third quartile, and the horizontal line dividing the

1148 box is the median. The whiskers represent the $10^{\text {th }}$ and $90^{\text {th }}$ percentiles. Two-way

1149 ANOVA followed by Sidak's multiple comparisons revealed that 7 retinal-fed FBS lines

1150 increase sleep significantly when stimulated with 627nm LEDs when compared with

1151 vehicle-fed flies. ${ }^{* *} \mathrm{P}<0.01,{ }^{* *} \mathrm{P}<0.001,{ }^{* * *} \mathrm{P}<0.0001, \mathrm{n}=21-39$ flies per genotype and

1152 condition.

1153 C) Box plots of locomotor activity counts per minute awake for retinal-fed flies presented

1154 in B. The bottom and top of each box represents the first and third quartile, and the

1155 horizontal line dividing the box is the median. The whiskers represent the $10^{\text {th }}$ and

$115690^{\text {th }}$ percentiles. Two-way repeated measures ANOVA followed by Sidak's multiple

1157 comparisons test found that for most sleep-promoting FBS lines, locomotor activity per

1158 awake time is not affected when the flies are stimulated with $627 \mathrm{~nm}$ LEDs while it is

1159 increased in FBS45>UAS-CsChrimson flies. ${ }^{* * *} \mathrm{P}<0.0001, \mathrm{n}=21-38$ flies per genotype.

1160 D) Box plots of daytime sleep bout duration (in minutes) for retinal-fed flies presented in

1161 B. The bottom and top of each box represents the first and third quartile, and the

1162 horizontal line dividing the box is the median. The whiskers represent the $10^{\text {th }}$ and

$116390^{\text {th }}$ percentiles. Two-way repeated measures ANOVA followed by Sidak's multiple

1164 comparisons indicate that daytime sleep bout duration is increased in 10 FBS lines

1165 expressing CsChrimson when stimulated with 627nm LEDs. ${ }^{*} \mathrm{P}<0.05,{ }^{* *} \mathrm{P}<0.01$,

$1166 * * * * \mathrm{P}<0.0001, \mathrm{n}=21-38$ flies per genotype. 
1167 E) Box plots of nighttime sleep bout duration (in minutes) for retinal-fed flies presented

1168 in B. The bottom and top of each box represents the first and third quartile, and the

1169 horizontal line dividing the box is the median. The whiskers represent the $10^{\text {th }}$ and

$117090^{\text {th }}$ percentiles. Two-way repeated measures ANOVA followed by Sidak's multiple

1171 comparisons indicate that nighttime sleep bout duration is increased in 6 FBS lines

1172 expressing CsChrimson when stimulated with 627nm LEDs. ${ }^{* * *} \mathrm{P}<0.0001, \mathrm{n}=21-38$ flies

1173 per genotype.

1175 Figure 4: The 23E10-GAL4 driver contains sleep-promoting neurons that are 1176 located in the VNC.

1177 A-D) Representative confocal stacks of a female VT020742-AD; 23E10-DBD>UAS-GFP 1178 brain $(A)$, brain sagittal view (B), VNC (C) and VNC sagittal view (D). The cell bodies 1179 panel in $\mathrm{C}$ is a magnified view of the metathoracic area of the VNC. Yellow arrows 1180 indicate TPN1 like processes and gray arrows "bowtie" processes. Green, anti-GFP; 1181 magenta, anti-nc82 (neuropile marker). $A=$ anterior, $P=$ posterior, $V=$ ventral and $D=$ 1182 dorsal.

1183 E-F) Representative confocal stacks of a female 30A08-AD; 23E10-DBD>UAS-GFP 1184 brain $(E)$ and VNC $(F)$. The cell bodies panel in $F$ is a magnified view of the 1185 metathoracic area of the VNC. Yellow arrows indicate TPN1 processes in the brain and 1186 VNC. Green, anti-GFP.

1187 G-J) Representative confocal stacks of a female VT013602-AD; 23E10-DBD>UAS-GFP 1188 brain $(\mathrm{G})$, brain sagittal view $(\mathrm{H}), \mathrm{VNC}(\mathrm{I})$ and $\mathrm{VNC}$ sagittal view $(\mathrm{J})$. The cell bodies 1189 panel in I is a magnified view of the metathoracic area of the VNC. Gray arrows indicate 1190 "bowtie" processes and cell bodies in I and J. Green, anti-GFP; magenta, anti-nc82 1191 (neuropile marker). $\mathrm{A}=$ anterior, $\mathrm{P}=$ posterior, $\mathrm{V}=$ ventral and $\mathrm{D}=$ dorsal.

$1192 \mathrm{~K})$ Box plots of total sleep change in \% for control (Empty-AD; 23E10-DBD>UAS1193 CsChrimson), VT020742-AD; 23E10-DBD>UAS-CsChrimson, 30A08-AD; 23E101194 DBD>UAS-CsChrimson and VT013602-AD; 23E10-DBD>UAS-CsChrimson vehicle-fed 1195 and retinal-fed female flies upon 627nm LED stimulation. The bottom and top of each 
box represents the first and third quartile, and the horizontal line dividing the box is the median. The whiskers represent the $10^{\text {th }}$ and $90^{\text {th }}$ percentiles. Two-way ANOVA followed by Sidak's multiple comparisons revealed that retinal-fed VT020742-AD; 23E10-DBD>UAS-CsChrimson and VT013602-AD; 23E10-DBD>UAS-CsChrimson flies increase sleep significantly when stimulated with $627 \mathrm{~nm}$ LEDs when compared with vehicle-fed flies. ${ }^{* *} P<0.01,{ }^{* * *} P<0.0001, n . s .=$ not significant. $n=13-34$ flies per genotype and condition.

L) Box plots of locomotor activity counts per minute awake for retinal-fed flies presented in $\mathrm{K}$. The bottom and top of each box represents the first and third quartile, and the horizontal line dividing the box is the median. The whiskers represent the $10^{\text {th }}$ and $90^{\text {th }}$ percentiles. Two-way repeated measures ANOVA followed by Sidak's multiple comparisons test shows that locomotor activity per awake time is not affected when the flies are stimulated with $627 \mathrm{~nm}$ LEDs. $n . s .=$ not significant. $n=15-34$ flies per genotype.

M) Box plots of daytime sleep bout duration (in minutes) for retinal-fed flies presented in $K$. The bottom and top of each box represents the first and third quartile, and the horizontal line dividing the box is the median. The whiskers represent the $10^{\text {th }}$ and $90^{\text {th }}$ percentiles. Two-way repeated measures ANOVA followed by Sidak's multiple comparisons indicates that daytime sleep bout duration is increased in retinal-fed VT020742-AD; 23E10-DBD>UAS-CsChrimson and VT013602-AD; 23E10-DBD>UASCsChrimson flies when stimulated with 627nm LEDs. ${ }^{* *} \mathrm{P}<0.01,{ }^{* * *} \mathrm{P}<0.0001$, n.s. $=$ not significant. $n=15-34$ flies per genotype.

N) Box plots of nighttime sleep bout duration (in minutes) for retinal-fed flies presented in $\mathrm{K}$. The bottom and top of each box represents the first and third quartile, and the horizontal line dividing the box is the median. The whiskers represent the $10^{\text {th }}$ and $90^{\text {th }}$ percentiles. Two-way repeated measures ANOVA followed by Sidak's multiple comparisons indicates that nighttime sleep bout duration is increased in retinal-fed VT020742-AD; 23E10-DBD>UAS-CsChrimson and VT013602-AD; 23E10-DBD>UASCsChrimson flies when stimulated with 627nm LEDs. ${ }^{* * *} \mathrm{P}<0.001,{ }^{* * *} \mathrm{P}<0.0001$, n.s. $=$ not significant. $\mathrm{n}=15-34$ flies per genotype. 
1226 Figure 5: Silencing VNC-SP neurons reduces sleep.

1227 A) and F) Sleep profile in minutes of sleep per hour for control (Empty-AD; 23E10-

1228 DBD>UAS-Kir2.1, blue line) and VNC-SP>Kir2.1 (VT013602-AD; 23E10-DBD>UAS-

1229 Kir2.1, red line) female (A) and male (F) flies.

1230 B) and G) Box plots of total sleep time (in minutes) for flies presented in $A$ and $F$, 1231 respectively. The bottom and top of each box represents the first and third quartile, and 1232 the horizontal line dividing the box is the median. The whiskers represent the $10^{\text {th }}$ and $123390^{\text {th }}$ percentiles. A two-tailed unpaired t test revealed that total sleep is significantly 1234 reduced in VNC-SP>Kir2.1 female flies compared to controls (B). A two-tailed Mann1235 Whitney $U$ test revealed that total sleep is significantly reduced in VNC-SP>Kir2. 1 male 1236 flies compared to controls $(G) .{ }^{* * *} P<0.0001 . n=58-60$ flies per genotype for females 1237 and $\mathrm{n}=48$ flies per genotype for males.

1238 C) and $\mathbf{H}$ ) Box plots of daytime sleep bout duration (in minutes) for flies presented in $A$ 1239 and F, respectively. The bottom and top of each box represents the first and third 1240 quartile, and the horizontal line dividing the box is the median. The whiskers represent 1241 the $10^{\text {th }}$ and $90^{\text {th }}$ percentiles. Two-tailed Mann-Whitney $U$ tests revealed that daytime 1242 sleep bout duration is significantly reduced in VNC-SP>Kir2.1 female $(\mathrm{C})$ and male $(\mathrm{H})$ 1243 flies compared to controls. ${ }^{*} \mathrm{P}<0.05,{ }^{* * *} \mathrm{P}<0.0001 . \mathrm{n}=58-60$ flies per genotype for 1244 females and $n=48$ flies per genotype for males.

1245 D) and I) Box plots of nighttime sleep bout duration (in minutes) for flies presented in $A$ 1246 and F, respectively. The bottom and top of each box represents the first and third 1247 quartile, and the horizontal line dividing the box is the median. The whiskers represent 1248 the $10^{\text {th }}$ and $90^{\text {th }}$ percentiles. Two-tailed Mann-Whitney $U$ tests revealed that nighttime 1249 sleep bout duration is significantly reduced in VNC-SP>Kir2.1 female (D) and male (I) 1250 flies compared to controls. ${ }^{* *} \mathrm{P}<0.001$. $\mathrm{n}=58-60$ flies per genotype for females and $\mathrm{n}=48$ 1251 flies per genotype for males.

1252 E) and J) Box plots of locomotor activity counts per minute awake for flies presented in 1253 A and F, respectively. The bottom and top of each box represents the first and third 1254 quartile, and the horizontal line dividing the box is the median. The whiskers represent 
1255 the $10^{\text {th }}$ and $90^{\text {th }}$ percentiles. Two-tailed Mann-Whitney $U$ tests revealed that activity per

1256 minute awake is significantly reduced in VNC-SP>Kir2.1 female (E) while it is

1257 unchanged in male $(\mathrm{J})$ flies compared to controls. ${ }^{* *} \mathrm{P}<0.001$, n.s. $=$ not significant. $\mathrm{n}=58$ -

125860 flies per genotype for females and $n=48$ flies per genotype for males.

$1259 \mathrm{~K})$ and $\mathbf{N}$ ) Sleep profile in minutes of sleep per hour for control (Empty-AD; 23E10-

$1260 D B D>U A S-S h i t s 1$ ) female $(\mathrm{K})$ and male $(\mathrm{N})$ flies at $22^{\circ} \mathrm{C}$ (blue line) and $32^{\circ} \mathrm{C}$ (red line).

1261 L) and 0$)$ Sleep profile in minutes of sleep per hour for VNC-SP>UAS-Shits1 female (L)

1262 and male $(\mathrm{O})$ flies at $22^{\circ} \mathrm{C}$ (blue line) and $32^{\circ} \mathrm{C}$ (red line).

1263 M) and P) Box plots of nighttime sleep change in \% for female flies presented in $\mathrm{K}$ and $\mathrm{L}$

1264 (M) and male flies presented in $\mathrm{N}$ and $\mathrm{O}$. The bottom and top of each box represents

1265 the first and third quartile, and the horizontal line dividing the box is the median. The

1266 whiskers represent the $10^{\text {th }}$ and $90^{\text {th }}$ percentiles. Two-tailed unpaired t tests revealed

1267 that VNC-SP>Shits1 flies lose significantly more sleep when transferred to $32^{\circ} \mathrm{C}$

1268 compared with controls in both males and females. ${ }^{*} \mathrm{P}<0.05 . \mathrm{n}=27-31$ flies per

1269 genotype for females and $n=31$ flies per genotype for males.

1270

1271 Figure 6: VNC-SP neurons are cholinergic.

1272 A-F) Representative confocal stacks focusing on the metathoracic ganglion of the VNC 1273 of female VT013602-AD; 23E10-DBD>UAS-GFP flies stained with antibodies to ChAT

1274 (A), VGlut (B), GABA (C), 5HT (D), TH (E) and Tdc2 (F). Gray arrows in A indicate

1275 colocalization of GFP and ChAT staining in VNC-SP neurons. Green, anti-GFP;

1276 magenta, anti-ChAT (A), anti-VGlut (B), anti-GABA (C), anti-5HT (D), anti-TH (E) and

1277 anti-Tdc2 (F).

1278 G) Sleep profile in minutes of sleep per hour for control (Empty-AD; 23E10-DBD>UAS-

1279 ChAT RNAi 60028, blue line) and VNC-SP>ChAT ${ }^{R N A i}(V T 013602-A D ; 23 E 10-D B D>U A S-$

1280 ChATRNAi 60028 , red line) female flies.

1281 H) Box plots of total sleep time (in minutes) for flies presented in G. The bottom and top 1282 of each box represents the first and third quartile, and the horizontal line dividing the 
1283 box is the median. The whiskers represent the $10^{\text {th }}$ and $90^{\text {th }}$ percentiles. A two-tailed 1284 unpaired t test revealed that $V N C-S P>C h A T^{R N A i}$ flies sleep significantly less than controls. ${ }^{* * * *} \mathrm{P}<0.0001 . \mathrm{n}=22-30$ flies per genotype.

1286 I) Box plots of daytime sleep bout duration (in minutes) for flies presented in $G$. The 1287 bottom and top of each box represents the first and third quartile, and the horizontal line 1288 dividing the box is the median. The whiskers represent the $10^{\text {th }}$ and $90^{\text {th }}$ percentiles. A 1289 two-tailed Mann-Whitney $U$ test revealed that VNC-SP>ChATRNAi flies daytime sleep 1290 bout duration is significantly reduced compared with controls. ${ }^{* * *} \mathrm{P}<0.0001 . \mathrm{n}=22-30$ 1291 flies per genotype.

1292 J) Box plots of nighttime sleep bout duration (in minutes) for flies presented in $\mathrm{G}$. The 1293 bottom and top of each box represents the first and third quartile, and the horizontal line 1294 dividing the box is the median. The whiskers represent the $10^{\text {th }}$ and $90^{\text {th }}$ percentiles. A 1295 two-tailed Mann-Whitney U test revealed that VNC-SP>ChATRNAi flies nighttime sleep 1296 bout duration is significantly reduced compared with controls. ${ }^{* * *} \mathrm{P}<0.0001 . \mathrm{n}=22-30$ 1297 flies per genotype.

1298 K) Box plots of locomotor activity counts per minute awake for flies presented in $G$. The 1299 bottom and top of each box represents the first and third quartile, and the horizontal line 1300 dividing the box is the median. The whiskers represent the $10^{\text {th }}$ and $90^{\text {th }}$ percentiles. A 1301 two-tailed Mann-Whitney $U$ test revealed that there is no difference in waking activity 1302 between VNC-SP>ChATRAi flies and controls. $\mathrm{n} . \mathrm{s}=$ not significant. $\mathrm{n}=22-30$ flies per 1303 genotype.

1304 L) Box plots of total sleep change in \% for vehicle-fed and retinal-fed VT013602-AD; 1305 23E10-DBD>UAS-Cs Chrimson; UAS-GFP.VALIUM10 (control) and VT013602-AD; 1306 23E10-DBD>UAS-Cs Chrimson; UAS-ChATRNAi 60028 flies stimulated by 627nm LEDs.

1307 The bottom and top of each box represents the first and third quartile, and the horizontal 1308 line dividing the box is the median. The whiskers represent the $10^{\text {th }}$ and $90^{\text {th }}$ percentiles. 1309 A two-way ANOVA followed by Sidak's multiple comparisons shows that sleep is 1310 significantly increased in control flies and that expressing ChAT RNAi in VNC-SP 1311 neurons completely abolishes the sleep-promoting effect of VNC-SP neurons activated 
1312

1313

1314

1315

1316

1317

1318

1319

1320

1321

1322

1323

1324

1325

1326

1327

1328

1329

1330

1331

1332

1333

1334

1335

1336

1337

1338

1339

1340

by $627 \mathrm{~nm}$ LEDs. ${ }^{* * * *} \mathrm{P}<0.0001$, n.s= not significant. $\mathrm{n}=31-36$ flies per genotype and condition.

\section{Figure 7: The 23E10-LexA driver does not express in VNC-SP neurons.}

A-E) Representative confocal stacks of a female 23E10-LexA>LexAop2-GFP; 23E10GAL4>UAS-RFP fly showing the brain (A), a magnified view of dFB neurons (B), the VNC (C), VNC-SP neurons projections in the brain (D) and TPN1 neurons processes in the VNC (E). Yellow arrows in B show dFB neurons that are labeled by both the 23E10LexA and 23E10-GAL4 drivers, while the gray arrow shows a neuron that is only labeled by 23E10-GAL4. Our analysis revealed that in these co-labelling experiments, 23E10LexA expresses in 13-20 dFB neurons while 23E10-GAL4 labels 19-26 dFB cells per brain. Importantly, all the neurons labeled by 23E10-LexA are part of the 23E10-GAL4 expression pattern. Yellow arrows in C show the TPN1 neurons and gray arrows the VNC-SP neurons. Green, anti-GFP; magenta, anti-RFP.

F) Box plots of total sleep change in \% for vehicle-fed and retinal-fed 23E10-GAL4/+ and UAS-CsChrimson/+ parental controls and 23E10-GAL4>UAS-CsChrimson female flies stimulated by $627 \mathrm{~nm}$ LEDs. The bottom and top of each box represents the first and third quartile, and the horizontal line dividing the box is the median. The whiskers represent the $10^{\text {th }}$ and $90^{\text {th }}$ percentiles. Two-way ANOVA followed by Sidak's multiple comparisons revealed that retinal-fed 23E10-GAL4>UAS-CsChrimson flies significantly increase sleep when stimulated with $627 \mathrm{~nm}$ LEDs. Both parental controls show no difference. ${ }^{* * * *} \mathrm{P}<0.0001, \mathrm{n} . \mathrm{s}=$ not significant. $\mathrm{n}=24-42$ flies per genotype and condition.

G) Box plots of total sleep change in \% for vehicle-fed and retinal-fed 23E10-LexA/+ and LexAop2-CsChrimson/+ parental controls and 23E10-LexA>LexAop2-CsChrimson female flies stimulated by $627 \mathrm{~nm}$ LEDs. The bottom and top of each box represents the first and third quartile, and the horizontal line dividing the box is the median. The whiskers represent the $10^{\text {th }}$ and $90^{\text {th }}$ percentiles. A two-way ANOVA followed by Sidak's multiple comparisons revealed that no differences in sleep following stimulation. n.s= not significant. $n=23-31$ flies per genotype and condition. 
1341 Figure 8: The 23E10-GAL4 contains two groups of sleep regulating neurons.

1342 A) Representative confocal stacks of a female 23E10-GAL4>UAS-GFP fly showing dFB 1343 neurons and VNC-SP neurons.

1344 B) Box plots of total sleep recovered in \% during the first $24 \mathrm{~h}$ following $12 \mathrm{~h}$ of sleep 1345 deprivation at night for 23E10-GAL4/+ and UAS-Kir2.1/+ parental controls and 23E101346 GAL4>UAS-Kir2.1 female flies. A one-way ANOVA followed by Tukey's multiple 1347 comparisons demonstrate that both parental controls have a bigger homeostatic sleep 1348 rebound than 23E10-GAL4>UAS-Kir2.1 female flies. * $\mathrm{P}<0.05$, n.s= not significant. $1349 \mathrm{n}=47-62$ flies per genotype.

1350 C) Box plots of total sleep recovered in \% during the first $24 \mathrm{~h}$ following $12 \mathrm{~h}$ of sleep 1351 deprivation at night for Empty-AD; 23E10-DBD>UAS-Kir2.1 and VT013602-AD; 23E10$1352 D B D>U A S-K i r 2.1$ female flies. A two-tailed unpaired test revealed that there is no 1353 difference in homeostatic sleep rebound. n.s= not significant. $n=24-29$ flies per 1354 genotype.

D) Box plots of total sleep recovered in \% during the first $24 \mathrm{~h}$ following $12 \mathrm{~h}$ of sleep 1356 deprivation at night for Empty-AD; 23E10-DBD>UAS-Kir2.1 and FBS4>UAS-Kir2.1 1357 female flies. A two-tailed unpaired t test revealed that FBS4>UAS-Kir2.1 flies have a 1358 significantly reduced homeostatic sleep rebound compared to controls. ${ }^{* *} \mathrm{P}<0.01$. $\mathrm{n}=15$ 135928 flies per genotype.

1361 Table Legends

1362 Table 1: Quantification of the number of cells labeled by the 23E10-GAL4 driver.

1363 Average number \pm SEM and range of dFB neurons, all brain neurons and VNC neurons 1364 labeled in 23E10-GAL4>UAS-GFP female flies. 
1367 Identification of the AD construct combined with 23E10-DBD in each FBS line. Average

1368 number \pm SEM and range of dFB neurons labeled by each line. Bowtie $(\mathrm{Y} / \mathrm{N})$ indicates

1369 whether "bowtie" processes are seen in the brain, $\mathrm{Y}=$ yes, $\mathrm{N}=$ no. Range of additional

1370 (non-dFB) neurons labeled in the brain of each line. Average number \pm SEM of VNC

1371 metathoracic cells labeled by each FBS line. TPN1 (Y/N) indicates whether TPN1

1372 neurons are present in the expression pattern, $\mathrm{Y}=$ yes, $\mathrm{N}=$ no. Range of additional cells

1373 in the VNC include any VNC neurons that is not TPN1 or "bowtie" like (VNC-SP).

1375 Video Legends

1376 Video 1: Brain of a 23E10-GAL4>UAS-GFP female.

1377 Video 2: VNC of a 23E10-GAL4>UAS-GFP female.

1378 Video 3: Brain of a FBS5>UAS-GFP female.

1379 Video 4: VNC of a FBS5>UAS-GFP female.

1380 Video 5: Brain of a VT013602AD-23E10-DBD (VNC-SP)>UAS-GFP female.

1381 Video 6: VNC of a VT013602AD-23E10-DBD (VNC-SP)>UAS-GFP female.

Supplemental Figure Legends

1384 Figure 2-figure supplement 1: Additional sleep profiles and confocal images of 1385 FBS lines.

1386 A-R) Sleep profile (left) and representative confocal stacks (right) for brain and VNC of 1387 female FBS>UAS-TrpA1; UAS-GFP for each FBS line not presented in Figure 2.

1388 Figure 2-figure supplement 2: Additional sleep parameters for FBS female flies 1389 thermogenetically activated.

1390 A) Box plots of daytime sleep bout duration in minutes for flies presented in Figure 2B.

1391 The bottom and top of each box represents the first and third quartile, and the horizontal 
1392 line dividing the box is the median. The whiskers represent the $10^{\text {th }}$ and $90^{\text {th }}$ percentiles.

1393 Two-way repeated measures ANOVA followed by Sidak's multiple comparisons test revealed that 9 FBS line show a significant increase in daytime sleep bout duration between $22^{\circ} \mathrm{C}$ and $31^{\circ} \mathrm{C}$. ${ }^{*} \mathrm{P}<0.05,{ }^{* *} \mathrm{P}<0.01,{ }^{* * *} \mathrm{P}<0.001,{ }^{* * * *} \mathrm{P}<0.0001, \mathrm{n}=30-51$ flies per genotype.

B) Box plots of nighttime sleep bout duration in minutes for flies presented in Figure 2B.

The bottom and top of each box represents the first and third quartile, and the horizontal line dividing the box is the median. The whiskers represent the $10^{\text {th }}$ and $90^{\text {th }}$ percentiles.

1400 Two-way repeated measures ANOVA followed by Sidak's multiple comparisons test revealed that control and most FBS line show a significant decrease in nighttime sleep bout duration between $22^{\circ} \mathrm{C}$ and $31^{\circ} \mathrm{C}$. Only 6 sleep-promoting FBS lines show no difference between $22^{\circ} \mathrm{C}$ and $31^{\circ} \mathrm{C}$. Dunnett's multiple comparisons reveal that for 5 of

1404 them, nighttime sleep bout duration at $31^{\circ} \mathrm{C}$ is significantly increased compared with 1405 Empty control flies (\# on figure). ${ }^{*} \mathrm{P}<0.05,{ }^{* *} \mathrm{P}<0.01,{ }^{* *} \mathrm{P}<0.001,{ }^{* * *} \mathrm{P}<0.0001$, n.s $=$ not significant. $n=30-51$ flies per genotype.

Figure 2-figure supplement 3: Thermogenetic activation in male flies.

A) Box plots of total sleep change in \% ((total sleep on day3-total sleep on day2/total sleep on day2) X 100) for male control (Empty-AD; 23E10-DBD) and 22 FBS lines expressing UAS-TrpA1; UAS-GFP. The bottom and top of each box represents the first and third quartile, and the horizontal line dividing the box is the median. The whiskers represent the $10^{\text {th }}$ and $90^{\text {th }}$ percentiles. The gray rectangle spanning the horizontal axis

1414 indicates the interquartile range of the control. Kruskal-Wallis ANOVA followed by

1415 Dunn's multiple comparisons revealed that 9 FBS lines increase sleep significantly more 1416 than control flies when transferred to $31^{\circ} \mathrm{C}$. ${ }^{*} \mathrm{P}<0.05,{ }^{* *} \mathrm{P}<0.01,{ }^{* * * *} \mathrm{P}<0.0001, \mathrm{n}=26-46$ 1417 flies per genotype.

1418 B) Box plots of locomotor activity counts per minute awake for flies presented in $A$. The 1419 bottom and top of each box represents the first and third quartile, and the horizontal line 1420 dividing the box is the median. The whiskers represent the $10^{\text {th }}$ and $90^{\text {th }}$ percentiles. 
1421 Two-way repeated measures ANOVA followed by Sidak's multiple comparisons test

1422 found that for 4 sleep-promoting FBS lines (FBS5, FBS45, FBS53 and FBS68)

1423 locomotor activity per awake time is increased while no differences are seen for the 1424 other 5 sleep-promoting lines between $22^{\circ} \mathrm{C}$ and $31^{\circ} \mathrm{C}$. ${ }^{*} \mathrm{P}<0.05,{ }^{* *} \mathrm{P}<0.01,{ }^{* * *} \mathrm{P}<0.001$, $1425 * * * * \mathrm{P}<0.0001, \mathrm{n}=26-46$ flies per genotype.

1426 C) Box plots of daytime sleep bout duration for flies presented in A. The bottom and top

1427 of each box represents the first and third quartile, and the horizontal line dividing the 1428 box is the median. The whiskers represent the $10^{\text {th }}$ and $90^{\text {th }}$ percentiles. Two-way 1429 repeated measures ANOVA followed by Sidak's multiple comparisons test found that for 14307 out of the 9 sleep-promoting FBS lines, daytime sleep bout duration is significantly 1431 increased at $31^{\circ} \mathrm{C}$ compared with $22^{\circ} \mathrm{C}$. ${ }^{*} \mathrm{P}<0.05,{ }^{* *} \mathrm{P}<0.01,{ }^{* *} \mathrm{P}<0.001,{ }^{* * *} \mathrm{P}<0.0001$, $1432 n=26-46$ flies per genotype.

1433 D) Box plots of nighttime sleep bout duration for flies presented in A. The bottom and 1434 top of each box represents the first and third quartile, and the horizontal line dividing the 1435 box is the median. The whiskers represent the $10^{\text {th }}$ and $90^{\text {th }}$ percentiles. Two-way 1436 repeated measures ANOVA followed by Sidak's multiple comparisons revealed that 1437 control and most FBS line show a significant decrease in nighttime sleep bout duration 1438 between $22^{\circ} \mathrm{C}$ and $31^{\circ} \mathrm{C} .5$ sleep-promoting FBS lines show no difference between $143922^{\circ} \mathrm{C}$ and $31^{\circ} \mathrm{C}$ (FBS5, FBS42, FBS53, FBS81 and FBS84) while FBS45 and FBS68 1440 show an increase in nighttime sleep bout duration at $31^{\circ} \mathrm{C}$. Dunnett's multiple 1441 comparisons reveal that for FBS5, FBS42, FBS45, FBS53, FBS68,FBS81 and FBS84, 1442 nighttime sleep bout duration at $31^{\circ} \mathrm{C}$ is significantly increased compared with Empty control flies (\# on figure). ${ }^{* *} \mathrm{P}<0.01,{ }^{* * *} \mathrm{P}<0.001,{ }^{* * * *} \mathrm{P}<0.0001, \mathrm{n}=26-46$ flies per 1444 genotype.

1446 Figure 3-figure supplement 1: Vehicle-fed sleep data for females in optogenetic 1447 experiments.

1448 A) Box plots of locomotor activity counts per minute awake for vehicle-fed flies 1449 presented in Figure 3B. The bottom and top of each box represents the first and third 
1450 quartile, and the horizontal line dividing the box is the median. The whiskers represent

1451 the $10^{\text {th }}$ and $90^{\text {th }}$ percentiles. Two-way repeated measures ANOVA followed by Sidak's

1452 multiple comparisons test found that all lines except FBS42 show no difference in

1453 locomotor activity per awake time when the flies are stimulated with 627nm LEDs.

$1454{ }^{* * *} \mathrm{P}<0.001 . \mathrm{n}=20-39$ flies per genotype.

1455 B) Box plots of daytime sleep bout duration for vehicle-fed flies presented in Figure 3B.

1456 The bottom and top of each box represents the first and third quartile, and the horizontal

1457 line dividing the box is the median. The whiskers represent the $10^{\text {th }}$ and $90^{\text {th }}$ percentiles.

1458 Two-way repeated measures ANOVA followed by Sidak's multiple comparisons test

1459 found that most vehicle-fed sleep-promoting lines show no difference in daytime sleep

1460 bout duration when the flies are stimulated with $627 \mathrm{~nm}$ LEDs. ${ }^{*} \mathrm{P}<0.05,{ }^{* *} \mathrm{P}<0.01$,

$1461{ }^{* * *} \mathrm{P}<0.001 . \mathrm{n}=20-39$ flies per genotype.

1462 C) Box plots of nighttime sleep bout duration for vehicle-fed flies presented in Figure 3B.

1463 The bottom and top of each box represents the first and third quartile, and the horizontal

1464 line dividing the box is the median. The whiskers represent the $10^{\text {th }}$ and $90^{\text {th }}$ percentiles.

1465 Two-way repeated measures ANOVA followed by Sidak's multiple comparisons test

1466 show no difference in nighttime sleep bout duration when vehicle-fed flies are stimulated

1467 with 627nm LEDs. n=20-39 flies per genotype.

1469 Figure 3-figure supplement 2: Optogenetic activation in males.

1470 A) Box plots of total sleep change in \% ((total sleep on day3-total sleep on day2/total 1471 sleep on day2) X 100) for control (Empty) and 22 experimental vehicle-fed and retinal-

1472 fed male flies expressing CsChrimson upon 627nm LED stimulation. The bottom and

1473 top of each box represents the first and third quartile, and the horizontal line dividing the

1474 box is the median. The whiskers represent the $10^{\text {th }}$ and $90^{\text {th }}$ percentiles. Two-way

1475 ANOVA followed by Sidak's multiple comparisons revealed that 9 retinal-fed FBS lines

1476 increase sleep significantly when stimulated with 627nm LEDs when compared with

1477 vehicle-fed flies. ${ }^{*} \mathrm{P}<0.05,{ }^{* *} \mathrm{P}<0.01,{ }^{* * * *} \mathrm{P}<0.0001 . \mathrm{n}=20-44$ flies per genotype and

1478 condition. 
1479 B) Box plots of locomotor activity counts per minute awake for retinal-fed flies presented

1480 in A. The bottom and top of each box represents the first and third quartile, and the

1481 horizontal line dividing the box is the median. The whiskers represent the $10^{\text {th }}$ and

$148290^{\text {th }}$ percentiles. Two-way repeated measures ANOVA followed by Sidak's multiple

1483 comparisons test found that for most sleep-promoting FBS lines, locomotor activity per

1484 awake time is not affected when the flies are stimulated with $627 \mathrm{~nm}$ LEDs while it is

1485 increased in FBS72>UAS-CsChrimson flies. * $\mathrm{P}<0.05, \mathrm{n}=24-44$ flies per genotype.

1486 C) Box plots of daytime sleep bout duration (in minutes) for retinal-fed flies presented in

1487 A. The bottom and top of each box represents the first and third quartile, and the

1488 horizontal line dividing the box is the median. The whiskers represent the $10^{\text {th }}$ and

$148990^{\text {th }}$ percentiles. Two-way repeated measures ANOVA followed by Sidak's multiple

1490 comparisons indicate that daytime sleep bout duration is increased in 12 FBS lines

1491 expressing CsChrimson when stimulated with 627nm LEDs. ${ }^{*} \mathrm{P}<0.05,{ }^{* * *} \mathrm{P}<0.001$,

$1492{ }^{* * * *} \mathrm{P}<0.0001, \mathrm{n}=24-44$ flies per genotype.

1493 D) Box plots of nighttime sleep bout duration (in minutes) for retinal-fed flies presented

1494 in A. The bottom and top of each box represents the first and third quartile, and the

1495 horizontal line dividing the box is the median. The whiskers represent the $10^{\text {th }}$ and

$149690^{\text {th }}$ percentiles. Two-way repeated measures ANOVA followed by Sidak's multiple

1497 comparisons indicate that nighttime sleep bout duration is increased in 7 FBS lines

1498 expressing CsChrimson when stimulated with 627nm LEDs. ${ }^{*} \mathrm{P}<0.05,{ }^{* *} \mathrm{P}<0.01$,

$1499 * * * * 2<0.0001, n=24-44$ flies per genotype.

1501 Figure 3-figure supplement 3: Vehicle-fed sleep data for males in optogenetic 1502 experiments.

1503 A) Box plots of locomotor activity counts per minute awake for vehicle-fed flies

1504 presented in Figure 3-figure supplement 2A. The bottom and top of each box represents

1505 the first and third quartile, and the horizontal line dividing the box is the median. The

1506 whiskers represent the $10^{\text {th }}$ and $90^{\text {th }}$ percentiles. Two-way repeated measures ANOVA

1507 followed by Sidak's multiple comparisons test found no difference in locomotor activity 
1508 per awake time when the flies are stimulated with $627 \mathrm{~nm}$ LEDs. $\mathrm{n}=20-40$ flies per 1509 genotype.

1510 B) Box plots of daytime sleep bout duration for vehicle-fed flies presented in Figure 31511 figure supplement 2A. The bottom and top of each box represents the first and third 1512 quartile, and the horizontal line dividing the box is the median. The whiskers represent 1513 the $10^{\text {th }}$ and $90^{\text {th }}$ percentiles. Two-way repeated measures ANOVA followed by Sidak's 1514 multiple comparisons test found that most vehicle-fed sleep-promoting lines show no 1515 difference in daytime sleep bout duration when the flies are stimulated with $627 \mathrm{~nm}$ 1516 LEDs. ${ }^{*} \mathrm{P}<0.05,{ }^{* *} \mathrm{P}<0.01,{ }^{* *} \mathrm{P}<0.001 . \mathrm{n}=20-40$ flies per genotype.

1517 C) Box plots of nighttime sleep bout duration for vehicle-fed flies presented in Figure 31518 figure supplement $2 \mathrm{~A}$. The bottom and top of each box represents the first and third 1519 quartile, and the horizontal line dividing the box is the median. The whiskers represent 1520 the $10^{\text {th }}$ and $90^{\text {th }}$ percentiles. Two-way repeated measures ANOVA followed by Sidak's 1521 multiple comparisons test show no difference in nighttime sleep bout duration when 1522 vehicle-fed flies are stimulated with 627nm LEDs. n=20-40 flies per genotype.

1524 Figure 4-figure supplement 1: Vehicle-fed sleep data for females in optogenetic activation of lines containing only VNC neurons.

1526 A) Box plots of locomotor activity counts per minute awake for vehicle-fed flies presented in Figure 4K. The bottom and top of each box represents the first and third quartile, and the horizontal line dividing the box is the median. The whiskers represent the $10^{\text {th }}$ and $90^{\text {th }}$ percentiles. Two-way repeated measures ANOVA followed by Sidak's multiple comparisons test found that locomotor activity per awake time is reduced in vehicle-fed VT020742-AD; 23E10-DBD>UAS-CsChrimson and VT013602-AD; 23E10-

$1532 D B D>U A S-C s C h r i m s o n$ flies that are stimulated with $627 \mathrm{~nm}$ LEDs. ${ }^{* *} \mathrm{P}<0.01$, ${ }^{* * *} \mathrm{P}<0.001$. n.s. $=$ not significant. $\mathrm{n}=13-32$ flies per genotype.

1534 B) Box plots of daytime sleep bout duration for vehicle-fed flies presented in Figure 4K. 1535 The bottom and top of each box represents the first and third quartile, and the horizontal 1536 line dividing the box is the median. The whiskers represent the $10^{\text {th }}$ and $90^{\text {th }}$ percentiles. 
1537 Two-way repeated measures ANOVA followed by Sidak's multiple comparisons test

1538 found no difference in daytime sleep bout duration when the flies are stimulated with 1539 627nm LEDs. n.s= not significant. n=13-32 flies per genotype.

1540 C) Box plots of nighttime sleep bout duration for vehicle-fed flies presented in Figure 4K.

1541 The bottom and top of each box represents the first and third quartile, and the horizontal

1542 line dividing the box is the median. The whiskers represent the $10^{\text {th }}$ and $90^{\text {th }}$ percentiles.

1543 Two-way repeated measures ANOVA followed by Sidak's multiple comparisons test

1544 show that nighttime sleep bout duration is not different when vehicle-fed flies are

1545 stimulated with $627 n m$ LEDs. n.s.= not significant. n=13-32 flies per genotype.

Figure 4-figure supplement 2: Optogenetic activation of lines containing only VNC neurons. Male data.

1549 A) Box plots of total sleep change in \% for control (Empty-AD; 23E10-DBD>UAS1550 CsChrimson), VT020742-AD; 23E10-DBD>UAS-CsChrimson, 30A08-AD; 23E10-

1551 DBD>UAS-CsChrimson and VT013602-AD; 23E10-DBD>UAS-CsChrimson vehicle-fed

1552 and retinal-fed male flies upon $627 \mathrm{~nm}$ LED stimulation. The bottom and top of each box

1553 represents the first and third quartile, and the horizontal line dividing the box is the

1554 median. The whiskers represent the $10^{\text {th }}$ and $90^{\text {th }}$ percentiles. Two-way ANOVA

1555 followed by Sidak's multiple comparisons revealed that retinal-fed VT020742-AD;

1556 23E10-DBD>UAS-CsChrimson and VT013602-AD; 23E10-DBD>UAS-CsChrimson flies

1557 increase sleep significantly when stimulated with 627nm LEDs when compared with

1558 vehicle-fed flies. ${ }^{* * *} \mathrm{P}<0.0001$, n.s. = not significant. $\mathrm{n}=18-40$ flies per genotype and

1559 condition.

1560 B) Box plots of locomotor activity counts per minute awake for retinal-fed flies presented 1561 in A. The bottom and top of each box represents the first and third quartile, and the 1562 horizontal line dividing the box is the median. The whiskers represent the $10^{\text {th }}$ and $156390^{\text {th }}$ percentiles. Two-way repeated measures ANOVA followed by Sidak's multiple 1564 comparisons test show that locomotor activity per awake time is increased in 1565 VT020742-AD; 23E10-DBD>UAS-CsChrimson flies and is not affected in the other 
genotypes when the flies are stimulated with $627 \mathrm{~nm}$ LEDs. ${ }^{* *} \mathrm{P}<0.05$, n.s. $=$ not significant. $n=21-40$ flies per genotype.

1568 C) Box plots of daytime sleep bout duration (in minutes) for retinal-fed flies presented in A. The bottom and top of each box represents the first and third quartile, and the horizontal line dividing the box is the median. The whiskers represent the $10^{\text {th }}$ and

$157190^{\text {th }}$ percentiles. Two-way repeated measures ANOVA followed by Sidak's multiple

1572 comparisons indicates that daytime sleep bout duration is increased in retinal-fed

1573 VT020742-AD; 23E10-DBD>UAS-CsChrimson and VT013602-AD; 23E10-DBD>UAS-

1574 CsChrimson flies when stimulated with 627nm LEDs. ${ }^{* * *} \mathrm{P}<0.0001$, n.s. $=$ not

1575 significant. $n=21-40$ flies per genotype.

1576 D) Box plots of nighttime sleep bout duration (in minutes) for retinal-fed flies presented

1577 in A. The bottom and top of each box represents the first and third quartile, and the

1578 horizontal line dividing the box is the median. The whiskers represent the $10^{\text {th }}$ and $157990^{\text {th }}$ percentiles. Two-way repeated measures ANOVA followed by Sidak's multiple 1580 comparisons indicates that nighttime sleep bout duration is increased in retinal-fed 1581 VT020742-AD; 23E10-DBD>UAS-CsChrimson and VT013602-AD; 23E10-DBD>UAS-

1582 CsChrimson flies when stimulated with 627nm LEDs. ${ }^{* * *} \mathrm{P}<0.0001$, n.s. $=$ not 1583 significant. $n=21-40$ flies per genotype.

1585 Figure 4-figure supplement 3: Vehicle-fed sleep data for males in optogenetic 1586 activation of lines containing only VNC neurons.

1587 A) Box plots of locomotor activity counts per minute awake for vehicle-fed flies 1588 presented in Figure 4-figure supplement 2A. The bottom and top of each box represents 1589 the first and third quartile, and the horizontal line dividing the box is the median. The 1590 whiskers represent the $10^{\text {th }}$ and $90^{\text {th }}$ percentiles. Two-way repeated measures ANOVA 1591 followed by Sidak's multiple comparisons test found that locomotor activity per awake 1592 time is reduced in vehicle-fed VT020742-AD; 23E10-DBD>UAS-CsChrimson and 1593 VT013602-AD; 23E10-DBD>UAS-CsChrimson flies that are stimulated with $627 \mathrm{~nm}$ 1594 LEDs. ${ }^{* *} \mathrm{P}<0.01$, n.s.= not significant. $\mathrm{n}=18-32$ flies per genotype. 
1595 B) Box plots of daytime sleep bout duration for vehicle-fed flies presented in Figure 4-

1596 figure supplement 2A. The bottom and top of each box represents the first and third 1597 quartile, and the horizontal line dividing the box is the median. The whiskers represent 1598 the $10^{\text {th }}$ and $90^{\text {th }}$ percentiles. Two-way repeated measures ANOVA followed by Sidak's 1599 multiple comparisons test found no difference in daytime sleep bout duration when the 1600 flies are stimulated with 627nm LEDs. $n . s=$ not significant. n=18-32 flies per genotype.

1601 C) Box plots of nighttime sleep bout duration for vehicle-fed flies presented in Figure 41602 figure supplement 2A. The bottom and top of each box represents the first and third 1603 quartile, and the horizontal line dividing the box is the median. The whiskers represent 1604 the $10^{\text {th }}$ and $90^{\text {th }}$ percentiles. Two-way repeated measures ANOVA followed by Sidak's 1605 multiple comparisons test show that nighttime sleep bout duration is not increased when 1606 vehicle-fed flies are stimulated with 627nm LEDs. $n . s=$ not significant. $n=18-32$ flies per 1607 genotype.

Figure 4-figure supplement 4: Additional data for TPN1 and VNC-SP neurons.

1610

1611

1612

1613

1614

1615

1616

1617

1618

1619

1620

1621

1622

1623

A) Representative confocal stacks of a female 30A08-LexA>LexAop2-GFP; VT020742$A D$; 23E10-DBD>UAS-RFP fly showing the brain, the VNC and a magnified view of the cell bodies in the metathoracic ganglion. Yellow arrows show the TPN1 neurons and their processes in the VNC and in the brain and gray arrows the VNC-SP neurons and their processes. TPN1 neurons are labeled by 30A08-LexA and VT020742-AD; 23E10DBD while VNC-SP neurons are only present in VT020742-AD; 23E10-DBD. Green, anti-GFP; magenta, anti-RFP.

B) Representative confocal stacks of a female 30A08-LexA>LexAop2-KZip ; VT020742$A D ; 23 E 10-D B D>U A S-G F P$ fly showing the brain and the VNC. The KZip ${ }^{+}$repressor effectively remove expression in the TPN1 neurons leaving only the VNC-SP neurons. Green, anti-GFP; magenta, anti-nc82 (neuropile marker).

C) Box plots of total sleep change in \% for Empty-AD; 23E10-DBD>UAS-CsChrimson, VT020742-AD; 23E10-DBD>UAS-CsChrimson, Empty-AD; 23E10-DBD>UASCsChrimson; 30A08-LexA> LexAop2-KZip ${ }^{+}$and VT020742-AD; 23E10-DBD>UAS- 
CsChrimson; 30A08-LexA> LexAop2-KZip ${ }^{+}$vehicle-fed and retinal-fed female flies upon $627 \mathrm{~nm}$ LED stimulation. The bottom and top of each box represents the first and third quartile, and the horizontal line dividing the box is the median. The whiskers represent the $10^{\text {th }}$ and $90^{\text {th }}$ percentiles. Two-way ANOVA followed by Sidak's multiple comparisons revealed that retinal-fed VT020742-AD; 23E10-DBD>UAS-CsChrimson and VT020742-AD; 23E10-DBD>UAS-CsChrimson; 30A08-LexA> LexAop2-KZip flies increase sleep significantly when stimulated with $627 \mathrm{~nm}$ LEDs when compared with vehicle-fed flies. Tukey's multiple comparisons demonstrate that there is no difference in total sleep change between retinal-fed VT020742-AD; 23E10-DBD>UAS-CsChrimson and VT020742-AD; 23E10-DBD>UAS-CsChrimson; 30A08-LexA> LexAop2-KZip + flies. ${ }^{* *} \mathrm{P}<0.01,{ }^{* * *} \mathrm{P}<0.0001, \mathrm{n} . \mathrm{s} .=$ not significant. $\mathrm{n}=17-31$ flies per genotype and condition.

D) Representative confocal stacks of a female VT020742-AD/ VT013602-AD; 23E10$D B D>U A S-G F P$ fly showing the brain and the VNC. This line labels the two TPN1 cells and the two VNC-SP neurons. Green, anti-GFP; magenta, anti-nc82 (neuropile marker).
E) Representative confocal stacks of a female VT013602-AD; VT020742-DBD>UASGFP fly showing the brain and the VNC. This line labels the two VNC-SP neurons and their processes in the brain (gray arrows). Green, anti-GFP; magenta, anti-nc82 (neuropile marker).

F) Sleep profile of control female (Empty-AD; Empty-DBD>UAS-TrpA1) at $22^{\circ} \mathrm{C}$ (blue line) and $31^{\circ} \mathrm{C}$ (red line).

G) Sleep profile of VT013602-AD; VT020742-DBD>UAS-TrpA1 female flies at $22^{\circ} \mathrm{C}$ (blue line) and $31^{\circ} \mathrm{C}$ (red line).

H) Box plots of total sleep change in \% for flies presented in F and G. The bottom and top of each box represents the first and third quartile, and the horizontal line dividing the box is the median. The whiskers represent the $10^{\text {th }}$ and $90^{\text {th }}$ percentiles. A two-tailed unpaired t test revealed that activating VT013602-AD; VT020742-DBD neurons significantly increases sleep compared with controls. ${ }^{* * *} \mathrm{P}<0.0001 . n=13-14$ flies per genotype. 
1653

1654

1655

1656

1657

1658

1659

1660

1661

1662

1663

1664

1665

1666

1667

1668

1669

1670

1671

1672

1673

1674

1675

1676

1677

1678

1679

1680

1681

Figure 4-figure supplement 5: Arousal threshold for VNC-SP>UAS-CsChrimson flies

Arousal threshold in vehicle-fed and retinal-fed VT013602-AD; 23E10-DBD>UASCsChrimson female flies. Percentage of flies awakened by a stimulus of increasing strength (1, 2 and 4 downward movements in the SNAP apparatus) with and without 627nm LEDs stimulation. Two-way ANOVA followed by Sidak's multiple comparisons indicates that in retinal-fed flies activation of VT013602-AD; 23E10-DBD neurons reduce the responsiveness to the 1 and 2 stimulus strength when compared with nonactivated flies. No difference in responsiveness is seen at the strongest stimulus (4). Two-way ANOVA followed by Sidak's multiple comparisons indicates that in vehicle-fed flies no difference in responsiveness is seen between LED stimulated and nonstimulated flies. ${ }^{* * * *} \mathrm{P}<0.0001$, n.s. = not significant. $\mathrm{n}=16$ flies per genotype and condition.

\section{Figure 6-figure supplement 1: Additional data demonstrating that VNC-SP} neurons are cholinergic.

A) Representative confocal stacks of a female ChAT-LexA>LexAop2-GFP; VT013602$A D$; $23 E 10-D B D(V N C-S P)>U A S-R F P$ focusing on the cell bodies in the metathoracic ganglion of the VNC. Gray arrows show the VNC-SP neurons that are co-labelled by the ChAT-LexA driver. Green, anti-GFP; magenta, anti-RFP.

B) Representative confocal stacks of a female ChAT-LexA>LexAop2-GFP; 23E10GAL4>UAS-RFP. Top panels, focusing on the cell bodies in the metathoracic ganglion of the VNC. Gray arrows show the VNC-SP neurons that are co-labelled by the ChATLexA driver. Bottom panels, focusing on dFB neurons. Red arrows show 23E10-GAL4 dFB neurons that are also labeled by ChAT-LexA. Green, anti-GFP; magenta, anti-RFP.

C) Quantification of ChAT knockdown efficiency by RNAi. qPCR was performed on the body and eyes of control flies (GMR-GAL4>+) and flies expressing ChAT RNAi (line 60028 ) in the eyes driven by GMR-GAL4 (GMR-GAL4>ChAT RNAi). Expression levels were normalized to control levels. Two-way ANOVA followed by Sidak's multiple 
comparisons revealed that in GMR-GAL4>ChAT RNAi flies ChAT levels are significantly reduced in the eyes but not in the body. ${ }^{* *} \mathrm{P}<0.01$. n.s. $=$ not significant. $n=3-4$ replicates per genotype.

D) ChAT immunostaining in VNC-SP>UAS-GFP (top panels) and VNC-SP>UAS-GFP; UAS-ChAT RNAi flies (bottom panels). Gray arrows point to neurons positive for GFP and ChAT. Green, anti-GFP; magenta, anti-ChAT.

E) Quantification of data presented in D. A one-tailed unpaired t test revealed that expressing ChAT RNAi in VNC-SP neurons significantly reduce ChAT levels as measured with ChAT antibody staining. ${ }^{*} P<0.05$. $n=14-17$ VNC analyzed per genotype.

F) Box plots of total sleep time (in minutes) for control (Empty-AD; 23E10-DBD>ChAT 60028 ) and VNC-SP>ChAT 60028 male flies. The bottom and top of each box represents the first and third quartile, and the horizontal line dividing the box is the median. The whiskers represent the $10^{\text {th }}$ and $90^{\text {th }}$ percentiles. A two-tailed MannWhitney $U$ test revealed that $V N C-S P>C h A T^{R N A i}$ male flies sleep significantly less than controls. ${ }^{* * *} \mathrm{P}<0.0001 . \mathrm{n}=24-30$ flies per genotype.

G) Box plots of daytime sleep bout duration (in minutes) for control (Empty-AD; 23E10$D B D>C h A T$ 60028) and VNC-SP>ChAT 60028 male flies. The bottom and top of each box represents the first and third quartile, and the horizontal line dividing the box is the median. The whiskers represent the $10^{\text {th }}$ and $90^{\text {th }}$ percentiles. A two-tailed MannWhitney $U$ test revealed that $V N C-S P>C h A T^{R N A i}$ male flies daytime sleep bout duration is significantly reduced compared with controls. ${ }^{* *} \mathrm{P}<0.001 . \mathrm{n}=24-30$ flies per genotype.

H) Box plots of nighttime sleep bout duration (in minutes) for control (Empty-AD; 23E10$D B D>C h A T$ 60028) and VNC-SP>ChAT 60028 male flies. The bottom and top of each box represents the first and third quartile, and the horizontal line dividing the box is the median. The whiskers represent the $10^{\text {th }}$ and $90^{\text {th }}$ percentiles. A two-tailed MannWhitney $U$ test revealed that $V N C-S P>C h A T^{R N A i}$ male flies nighttime sleep bout duration is significantly reduced compared with controls. ${ }^{* * *} \mathrm{P}<0.001$. $\mathrm{n}=24-30$ flies per genotype. 
1710 I) Box plots of locomotor activity counts per minute awake for control (Empty-AD;

$171123 E 10-D B D>C h A T$ 60028) and VNC-SP>ChAT 60028 male flies. The bottom and top of

1712 each box represents the first and third quartile, and the horizontal line dividing the box is

1713 the median. The whiskers represent the $10^{\text {th }}$ and $90^{\text {th }}$ percentiles. A two-tailed unpaired

$1714 \mathrm{t}$ test revealed that there is no difference in waking activity between VNC-SP>ChATRNAi

1715 male flies and controls. $n . s=$ not significant. $n=24-30$ flies per genotype.

1716 J) Box plots of total sleep change in \% for VT013602-AD; 23E10-DBD>UAS-

1717 CsChrimson; 2x LexAop2KZip ${ }^{+}$(control, no LexA driver) and for VT013602-AD; 23E10-

1718 DBD>UAS-CsChrimson; ChAT-LexA>2x LexAop2KZip ${ }^{+}$vehicle-fed and retinal-fed

1719 female flies upon 627nm LED stimulation. The bottom and top of each box represents

1720 the first and third quartile, and the horizontal line dividing the box is the median. The

1721 whiskers represent the $10^{\text {th }}$ and $90^{\text {th }}$ percentiles. Two-way ANOVA followed by Sidak's

1722 multiple comparisons revealed that retinal-fed VT013602-AD; 23E10-DBD>UAS-

1723 CsChrimson; ChAT-LexA>2x LexAop2KZip flies do not increase sleep compared with

1724 vehicle-fed flies when stimulated with 627nm LEDs. Control flies increase sleep

1725 significantly. ${ }^{* * *} \mathrm{P}<0.0001$, n.s. $=$ not significant. $\mathrm{n}=16-27$ flies per genotype and

1726 condition.

1727 K) Representative confocal stacks of VT013602-AD; 23E10-DBD>UAS-GFP (left) and 1728 VT013602-AD; 23E10-DBD>UAS-GFP; ChAT-LexA>2x LexAop2KZip + (right) female

1729 flies. Expression of GFP in VNC-SP neurons and in their bowtie brain processes is

1730 completely abolished by the expression of the KZip ${ }^{+}$repressor. Gray arrows show VNC-

1731 SP neurons. Green, anti-GFP; magenta, anti-RFP.

1732 L) Box plots of total sleep change in \% for Empty-AD; 23E10-DBD>UAS-CsChrimson;

1733 ChAT-LexA> LexAop2KZip ${ }^{+}$and for FBS45>UAS-CsChrimson; ChAT-LexA>

1734 LexAop2KZip ${ }^{+}$vehicle-fed and retinal-fed female flies upon 627nm LED stimulation. The

1735 bottom and top of each box represents the first and third quartile, and the horizontal line

1736 dividing the box is the median. The whiskers represent the $10^{\text {th }}$ and $90^{\text {th }}$ percentiles.

1737 Two-way ANOVA followed by Sidak's multiple comparisons revealed no differences in

1738 sleep when flies are stimulated with 627nm LEDs. n.s.= not significant. $n=22-29$ flies per

1739 genotype and condition. 
1740 M) Representative confocal stacks of FBS45>UAS-GFP (left) and FBS45>UAS-GFP;

1741 ChAT-LexA>LexAop2KZip+ (right) female flies. Expression of GFP in VNC-SP neurons

1742 and in their bowtie brain processes is completely abolished by the expression of the

$1743 \mathrm{KZip}{ }^{+}$repressor. Gray arrows show VNC-SP neurons. In addition, the KZip ${ }^{+}$repressor

1744 also consistently remove expression in 4-6 dFB neurons. On average $19.33 \pm 0.62 \mathrm{dFB}$

1745 neurons are labeled in FBS45>UAS-GFP; ChAT-LexA>LexAop2KZip ${ }^{+}$while it is more

1746 than 25 without the KZip ${ }^{+}$repressor (Table 2). Green, anti-GFP; magenta, anti-RFP.

1748 Figure 6-figure supplement 2: VNC-SP neurons are well positioned to integrate 1749 sensory inputs in the VNC and send this information to the brain.

1750 Representative confocal stacks of a female VT013602-AD; 23E10-DBD> UAS-

1751 5xDenMark-V5; UAS-syt.eGFP. Top panels, focusing on the brain "bowtie" processes.

1752 Middle panels, the VNC and bottom panels, a magnified view of the cell bodies area.

1753 Green, anti-GFP; magenta, anti-V5.

1754

1755

1756 
Figure 1

A

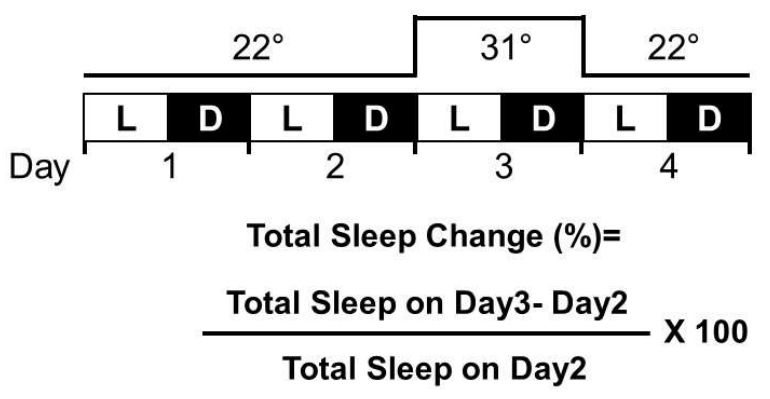

C

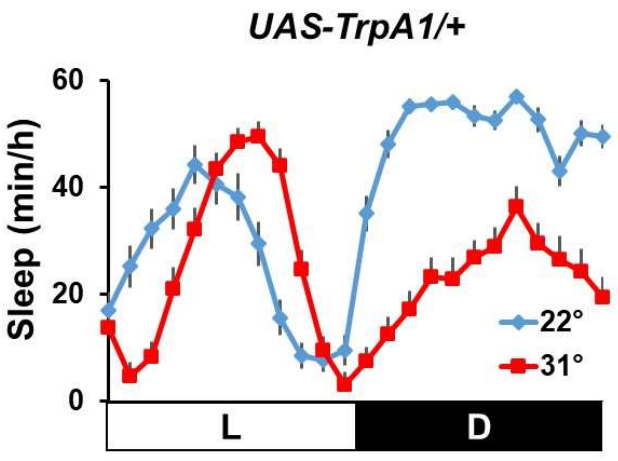

E

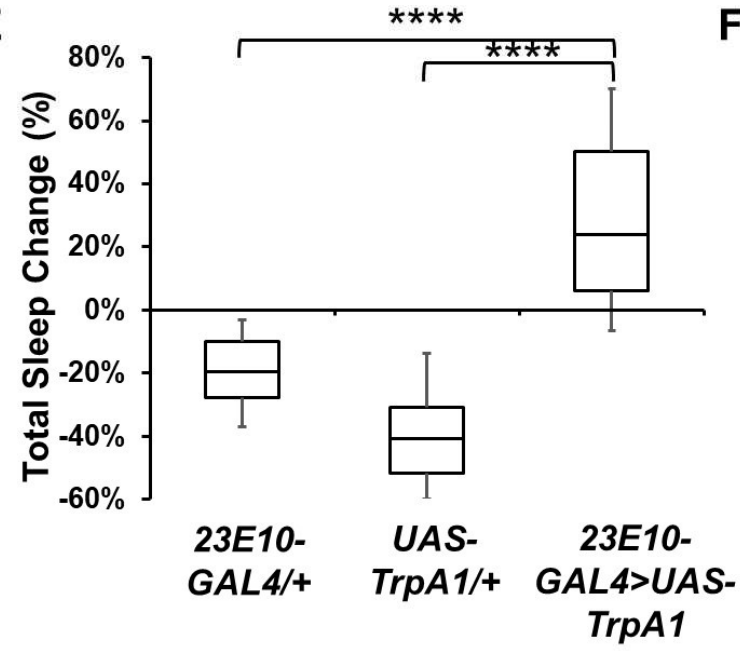

B

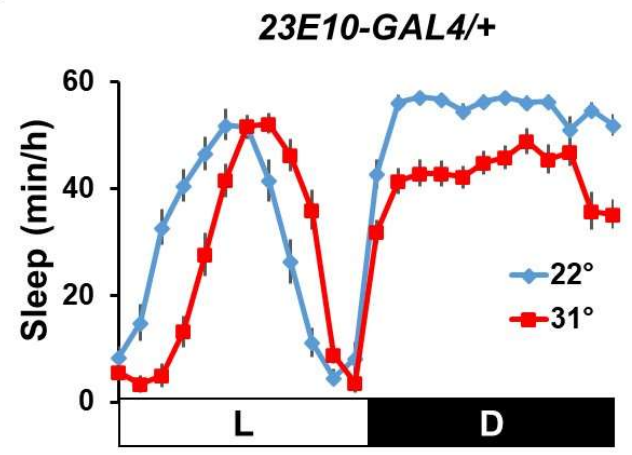

D

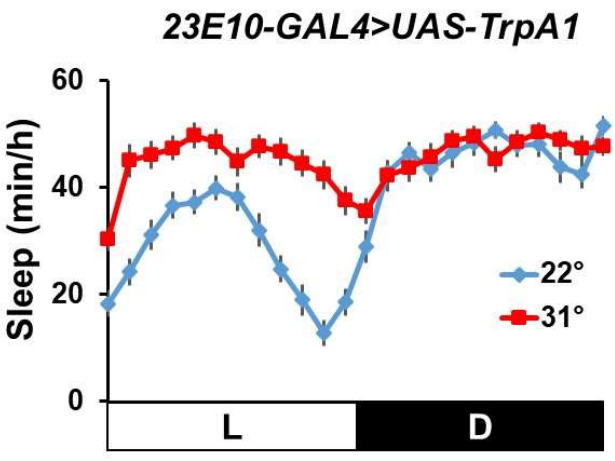

F

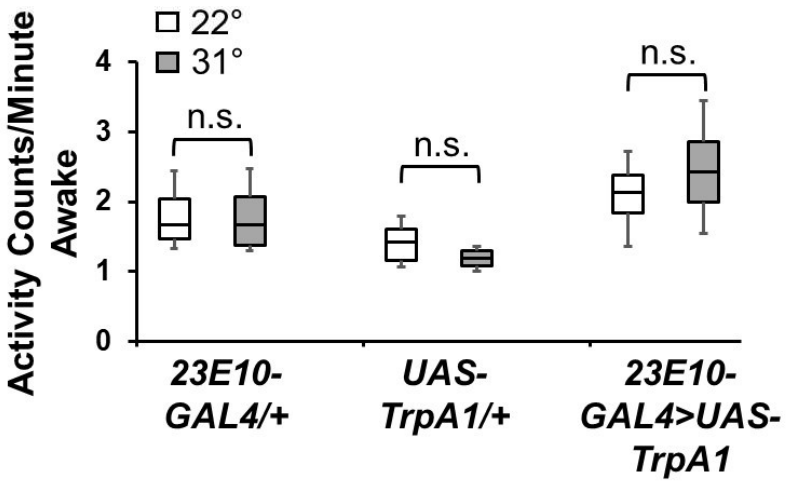

23E10-GAL4>UAS-mCD8GFP

G

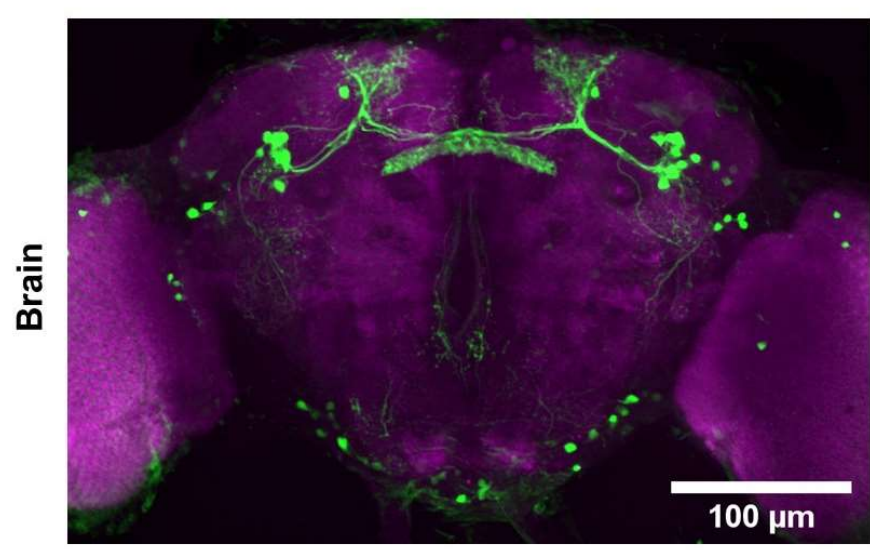

$\mathrm{H}$

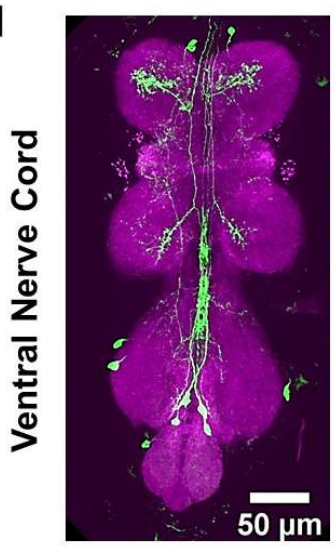


bioRxiv preprint doi: https://doi.org/10.1101/2022.02.02.478882; this version posted February 9, 2022. The copyright holder for this preprint (which was not certified by peer review) is the author/funder, who has granted bioRxiv a license to display the preprint in perpetuity. It is made available under aCC-BY-NC-ND 4.0 International license.

\section{Figure 2}

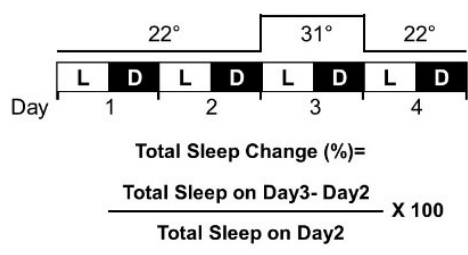

C

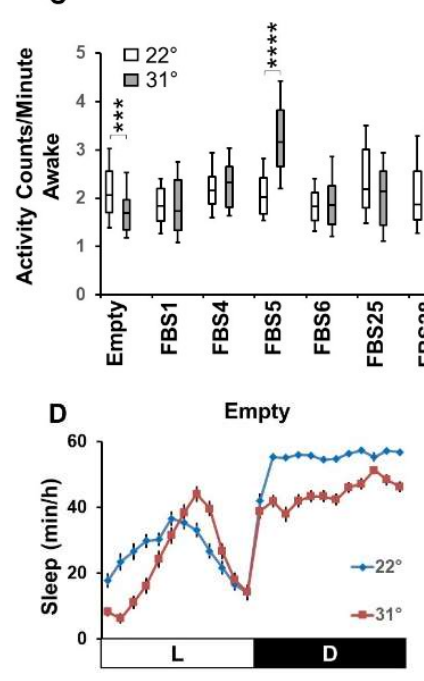

E

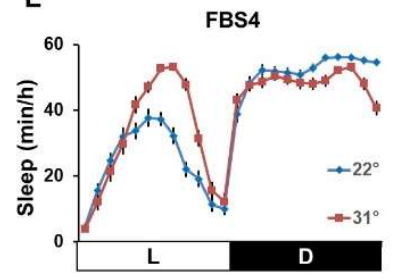

F

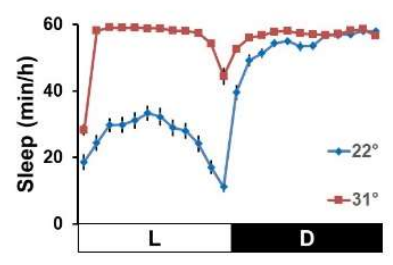

G
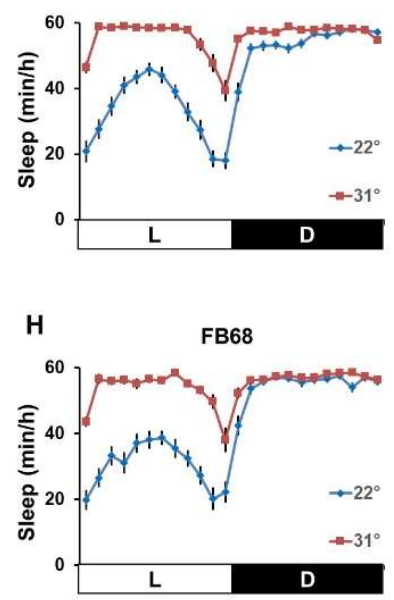

B

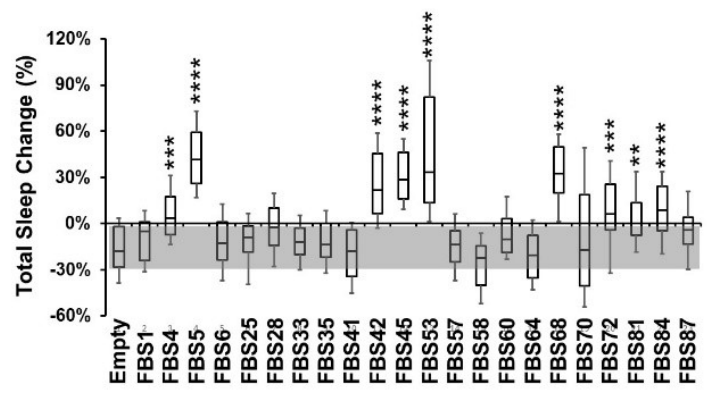


bioRxiv preprint doi: https://doi.org/10.1101/2022.02.02.478882; this version posted February 9, 2022. The copyright holder for this preprint (which was not certified by peer review) is the author/funder, who has granted bioRxiv a license to display the preprint in perpetuity. It is made za ailable under aCC-BY-NC-NDehialtaperaberiontablicense.

\section{Figure 3}
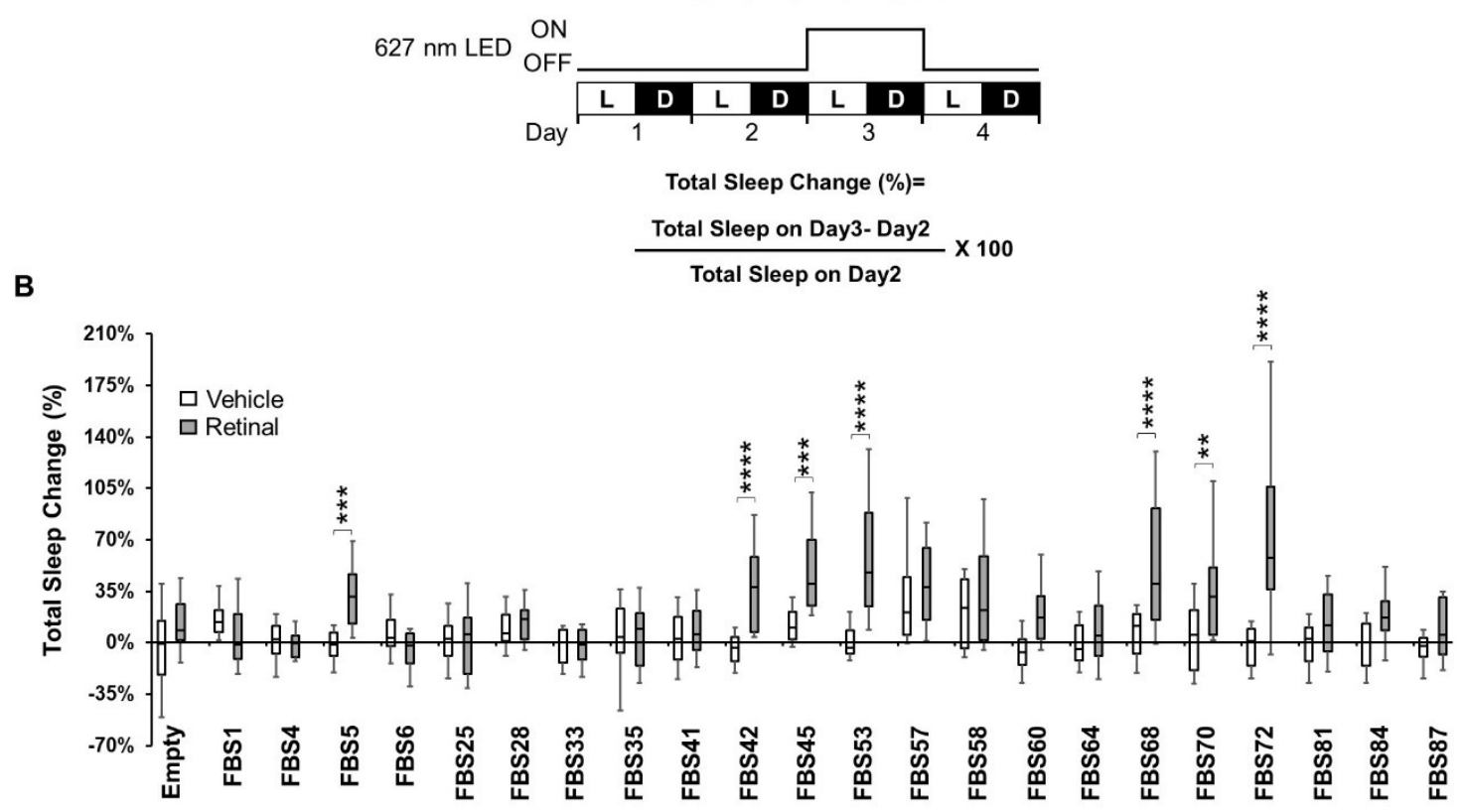

C

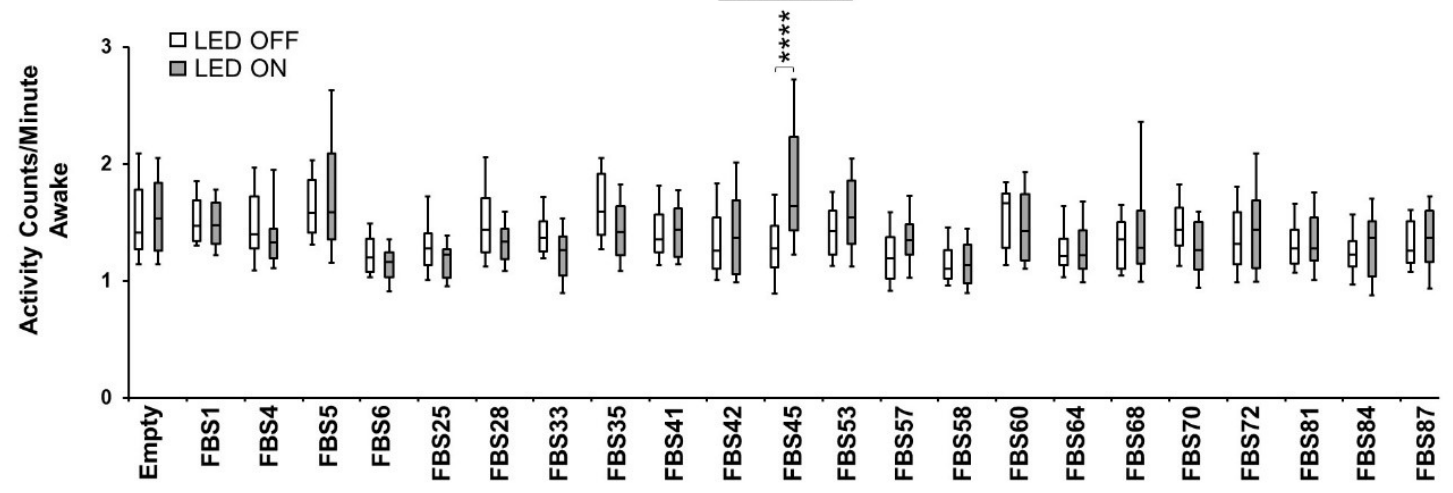

D

$\underline{\text { Retinal-Fed }}$

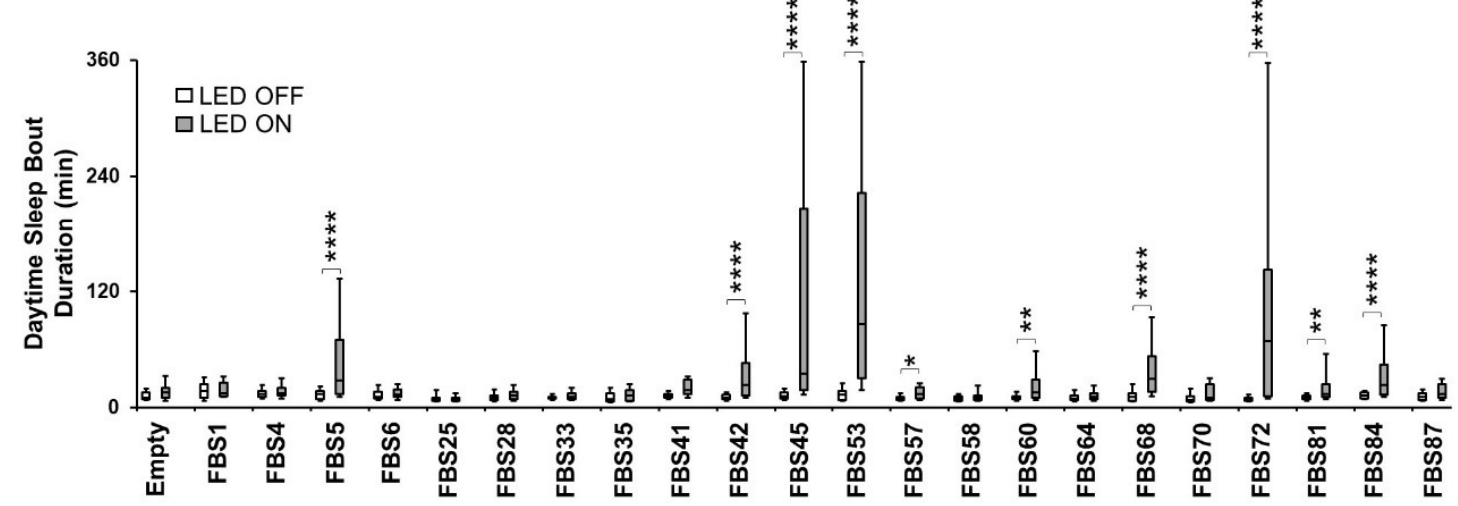

E

Retinal-Fed

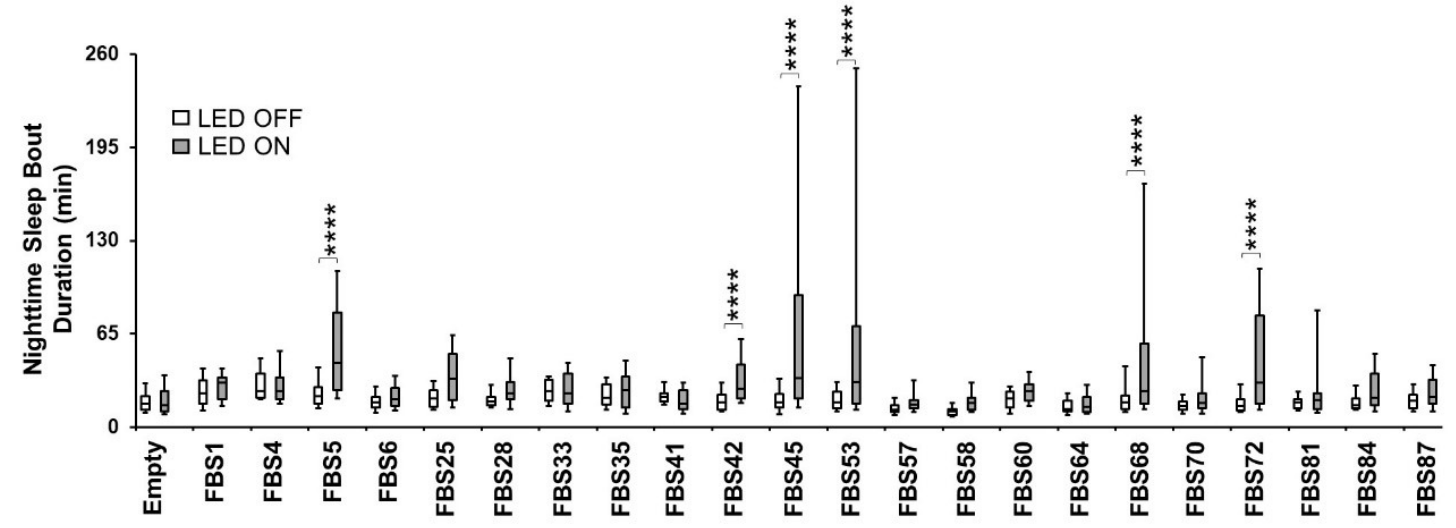


bioRxiv preprint doi: https://doi.org/10.1101/2022.02.02.478882; this version posted February 9, 2022. The copyright holder for this preprint (which was not certified by peer review) is the author/funder, who has granted bioRxiv a license to display the preprint in perpetuity. It is made

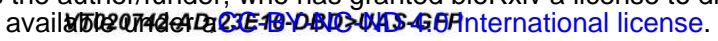

\section{Figure 4}
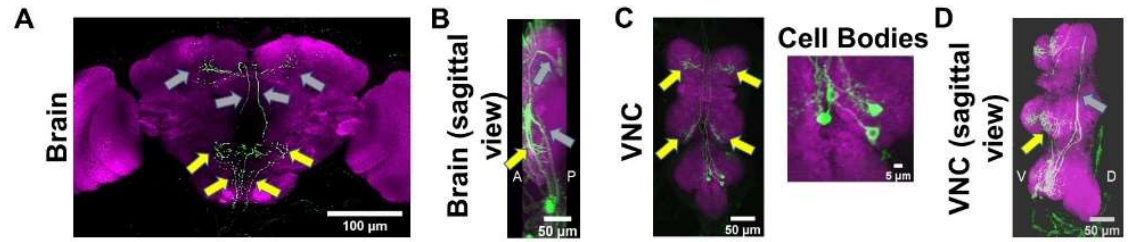

E
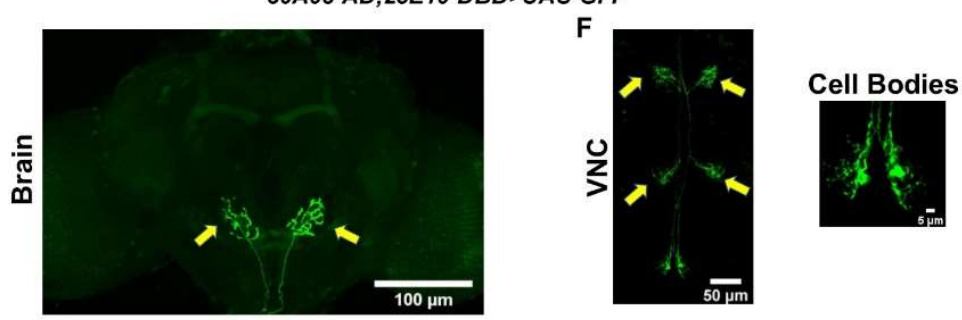

G
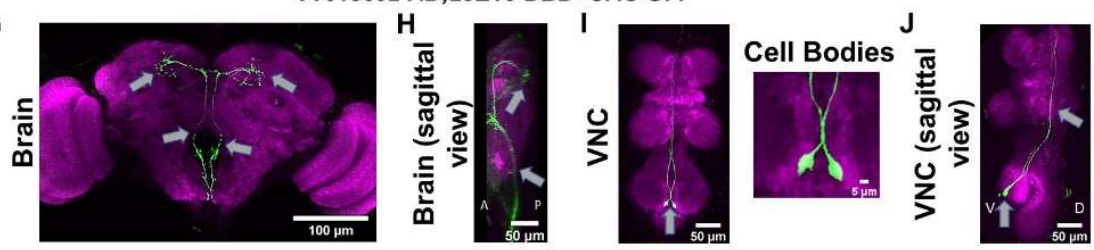

K

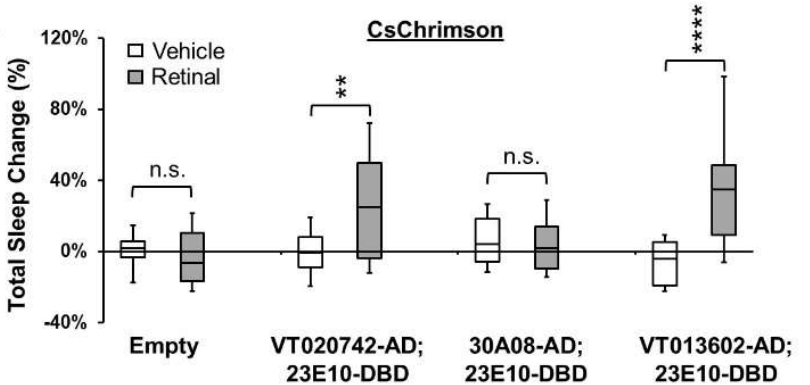

L

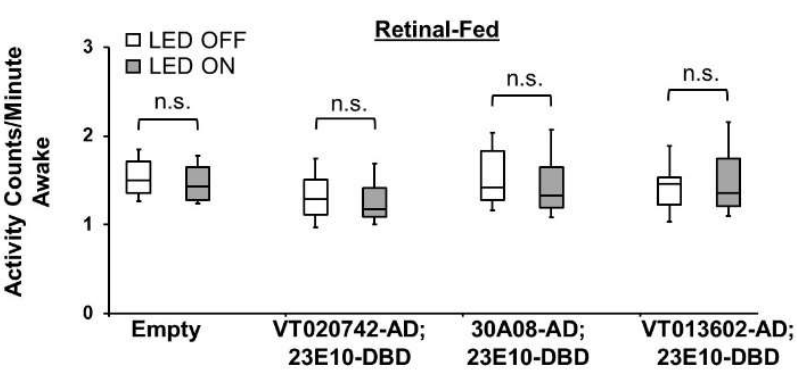

M

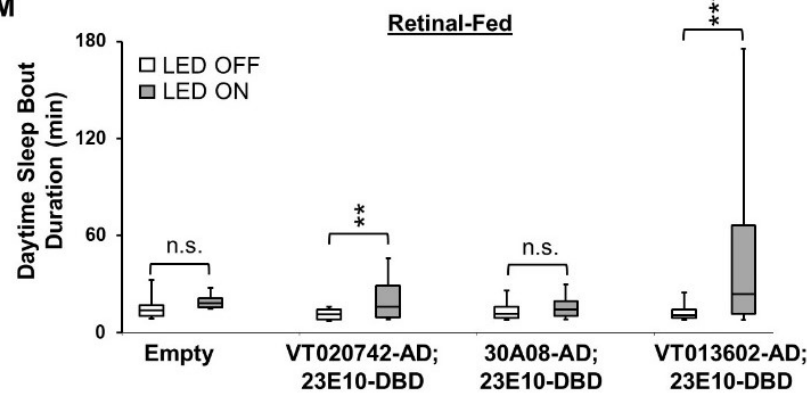

N

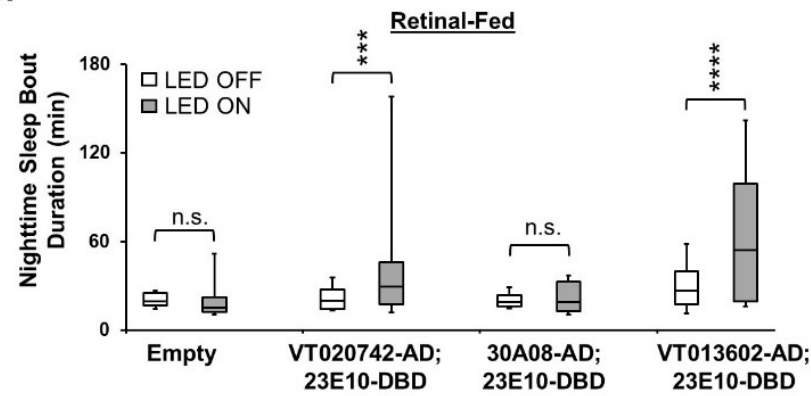


bioRxiv preprint doi: https://doi.org/10.1101/2022.02.02 478882; this version posted February 9,2022 . The copyright holder for this preprint (which was not certified by peer review) is the author/funder, who has granted bioRxiv a license to display the preprint in perpetuity. It is made available under aCC-BY-NC-ND 4.0 International license.

\section{Figure 5}

\section{Chronic silencing-Kir2.1}

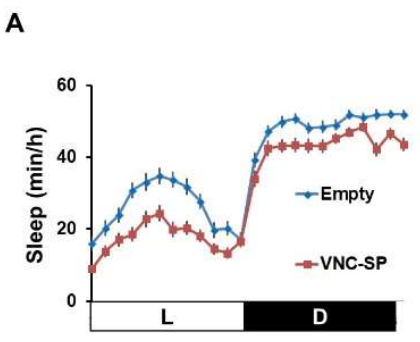

Females
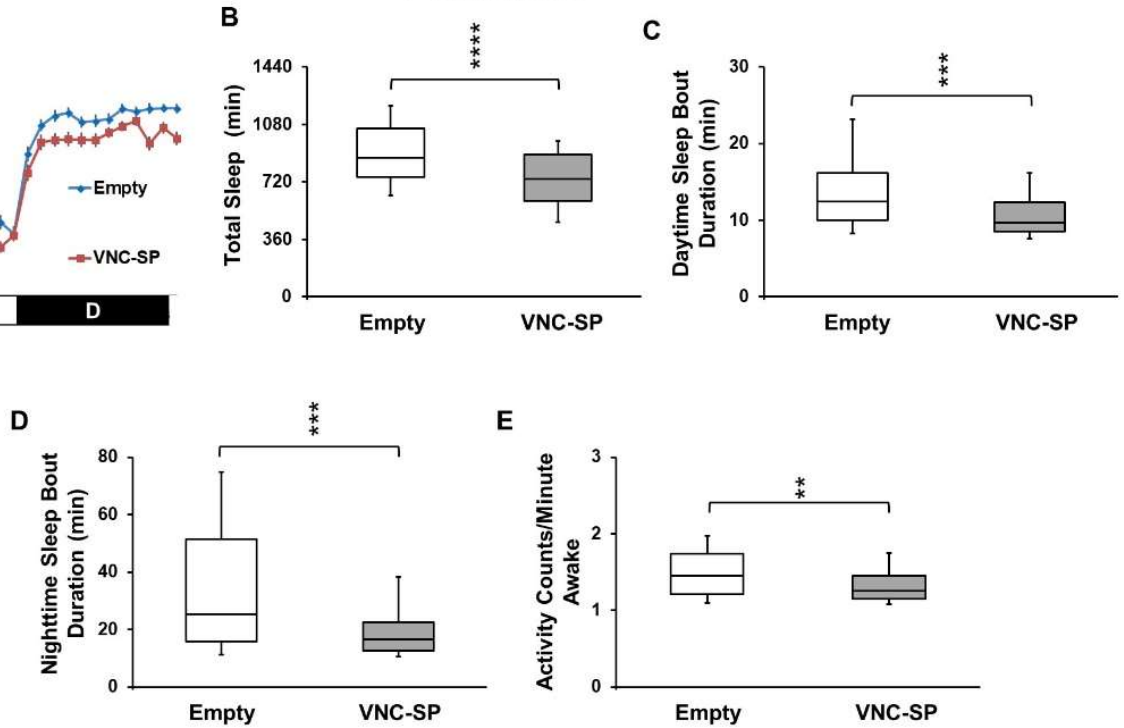

\section{Males}
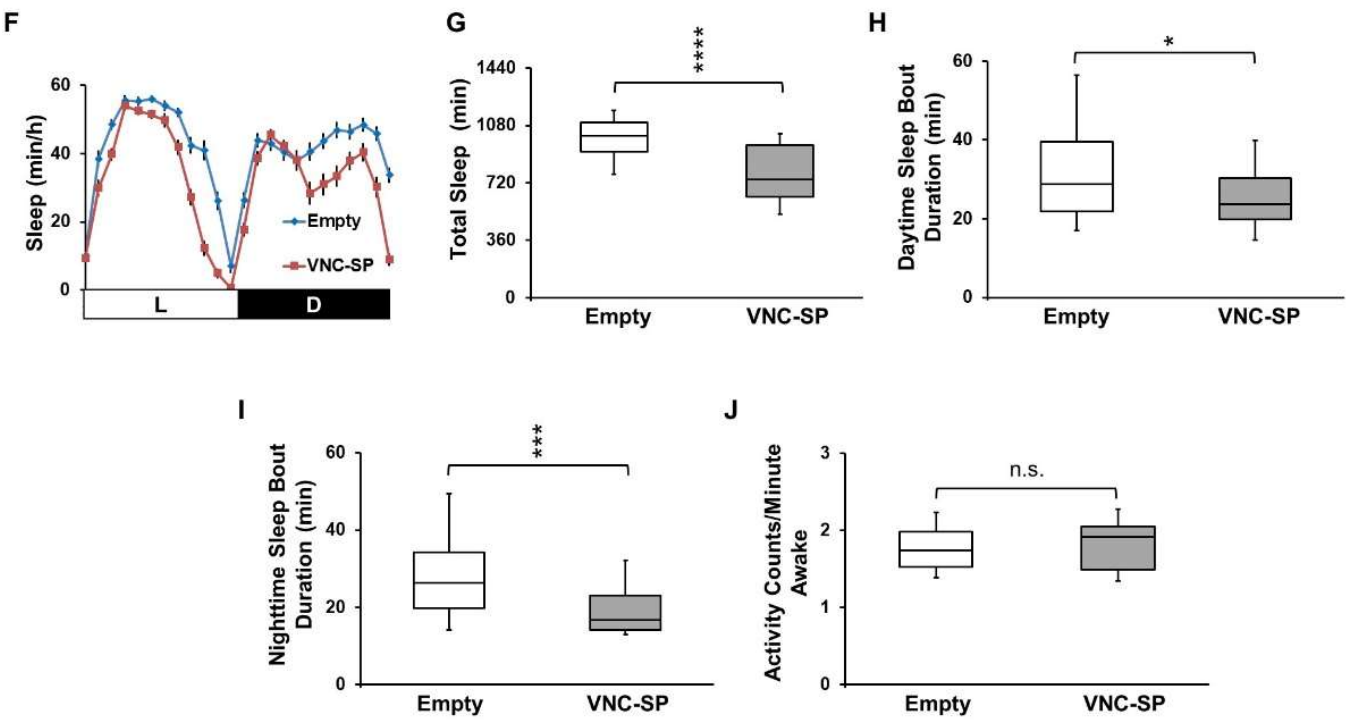

\section{Acute silencing-Shibire ${ }^{\text {ts1 }}$}

$\mathbf{K}$

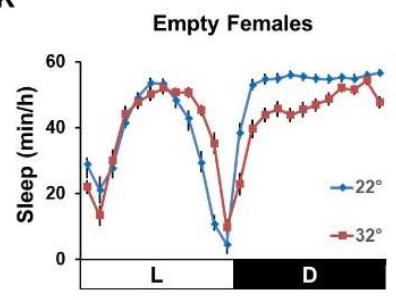

N

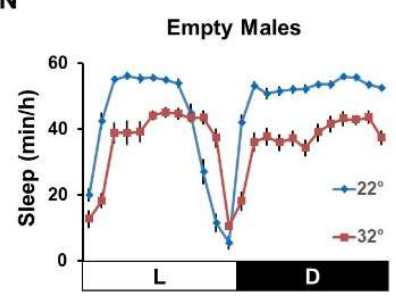

L

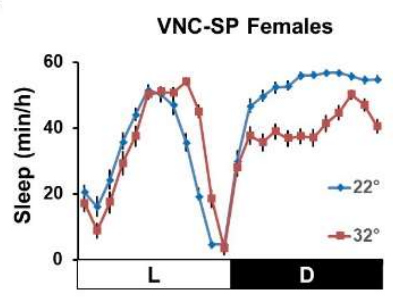

0

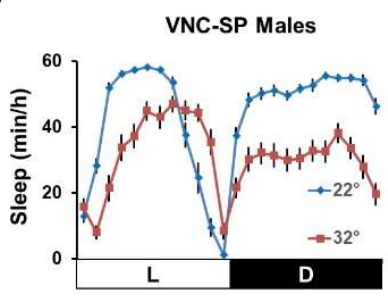

M

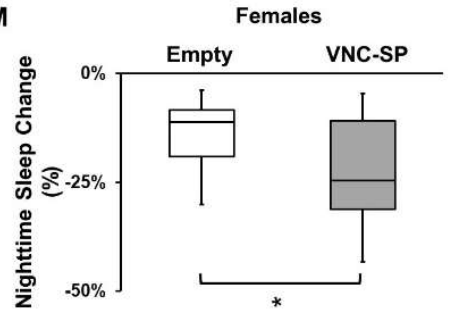

$\mathbf{P}$

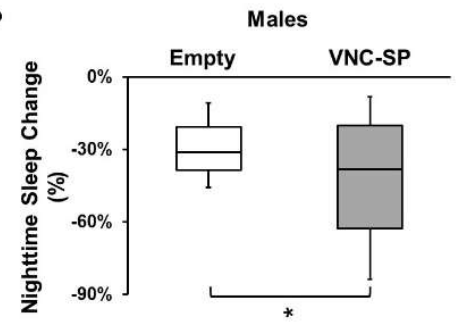




\section{Figure 6}

\section{VT013602-AD;23E10-DBD>UAS-GFP}

A
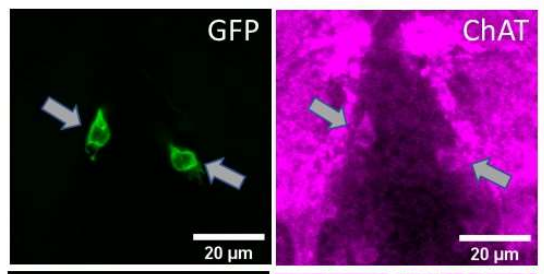

B
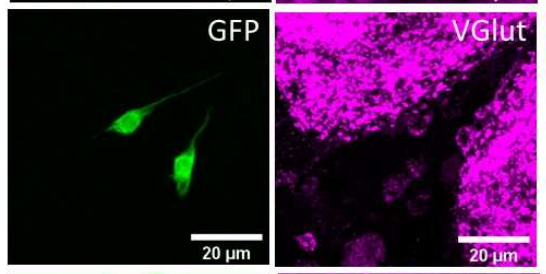

C
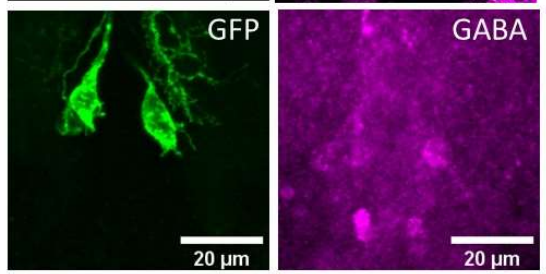

G

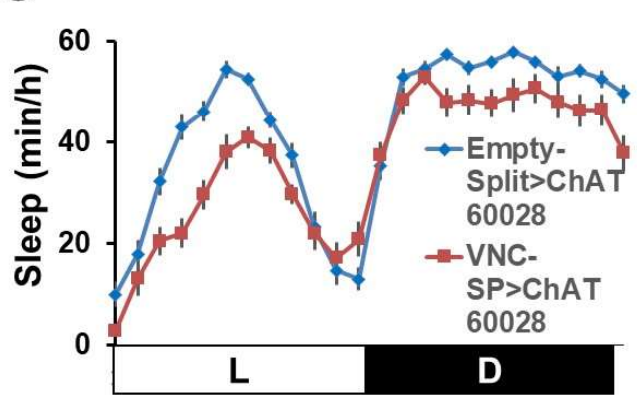

H

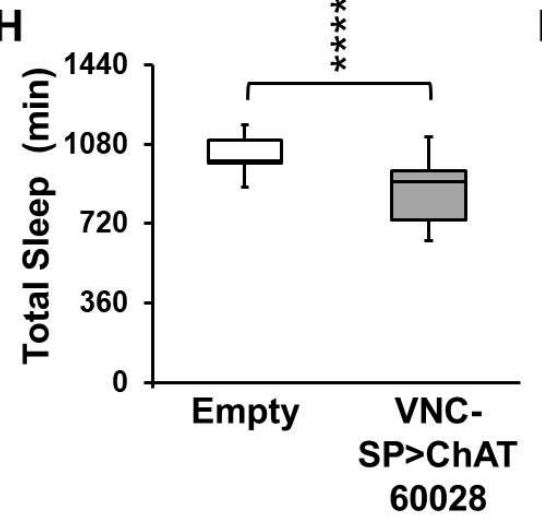

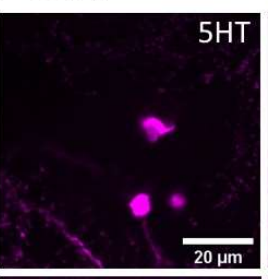
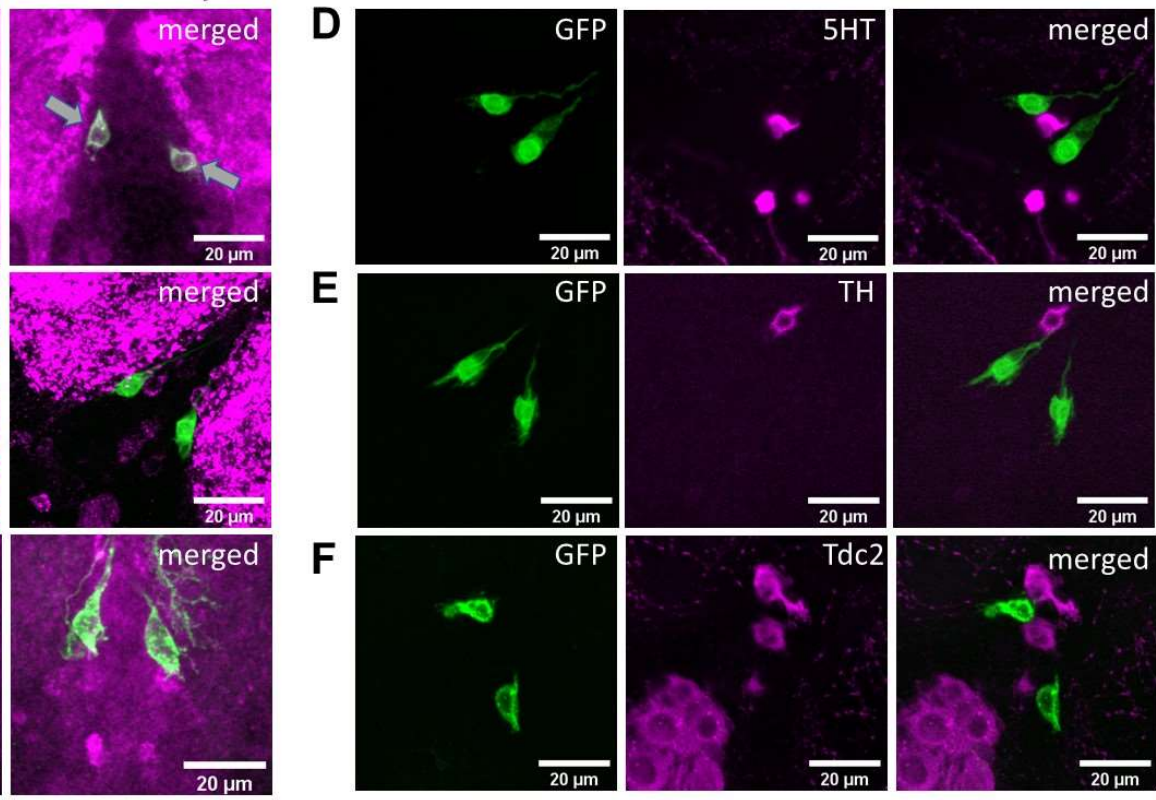

E

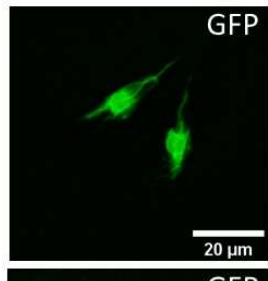

F
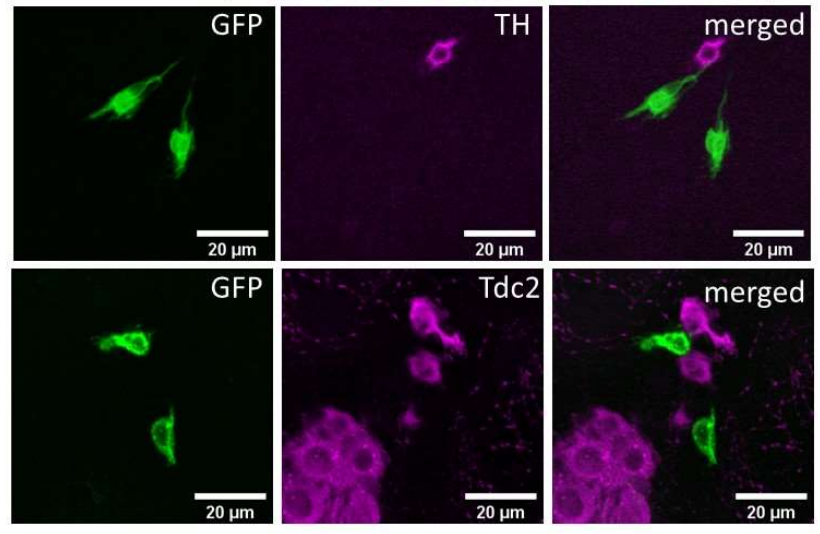

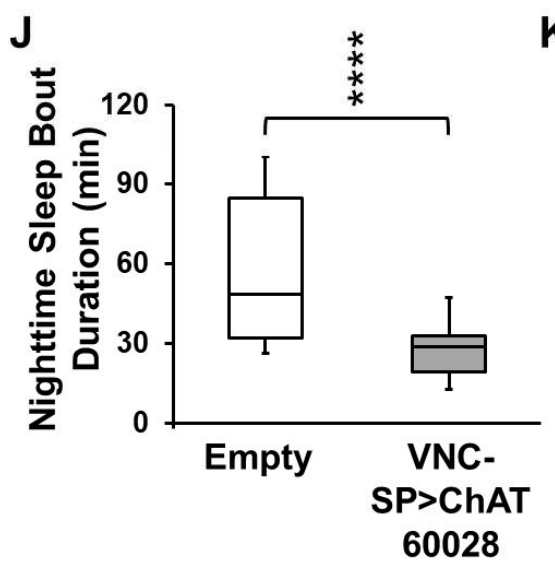

K

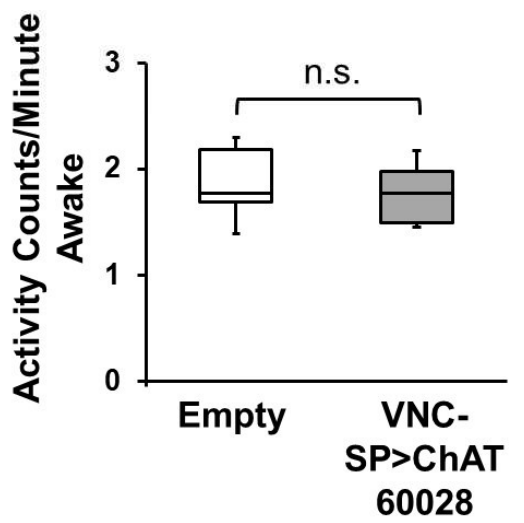

L

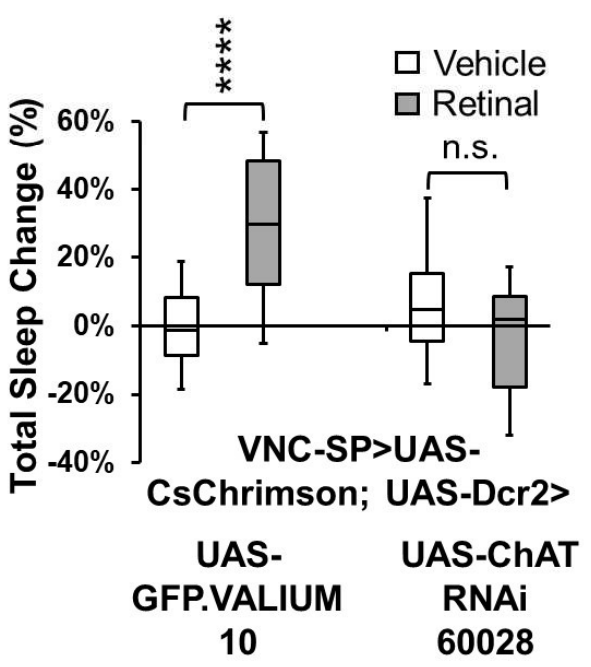




\section{Figure 7}

A

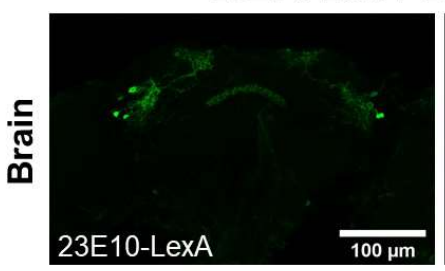

B

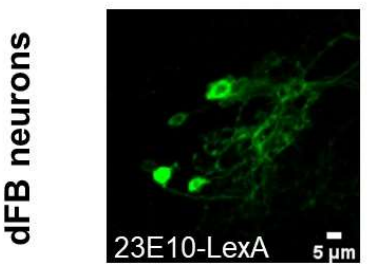

C

D
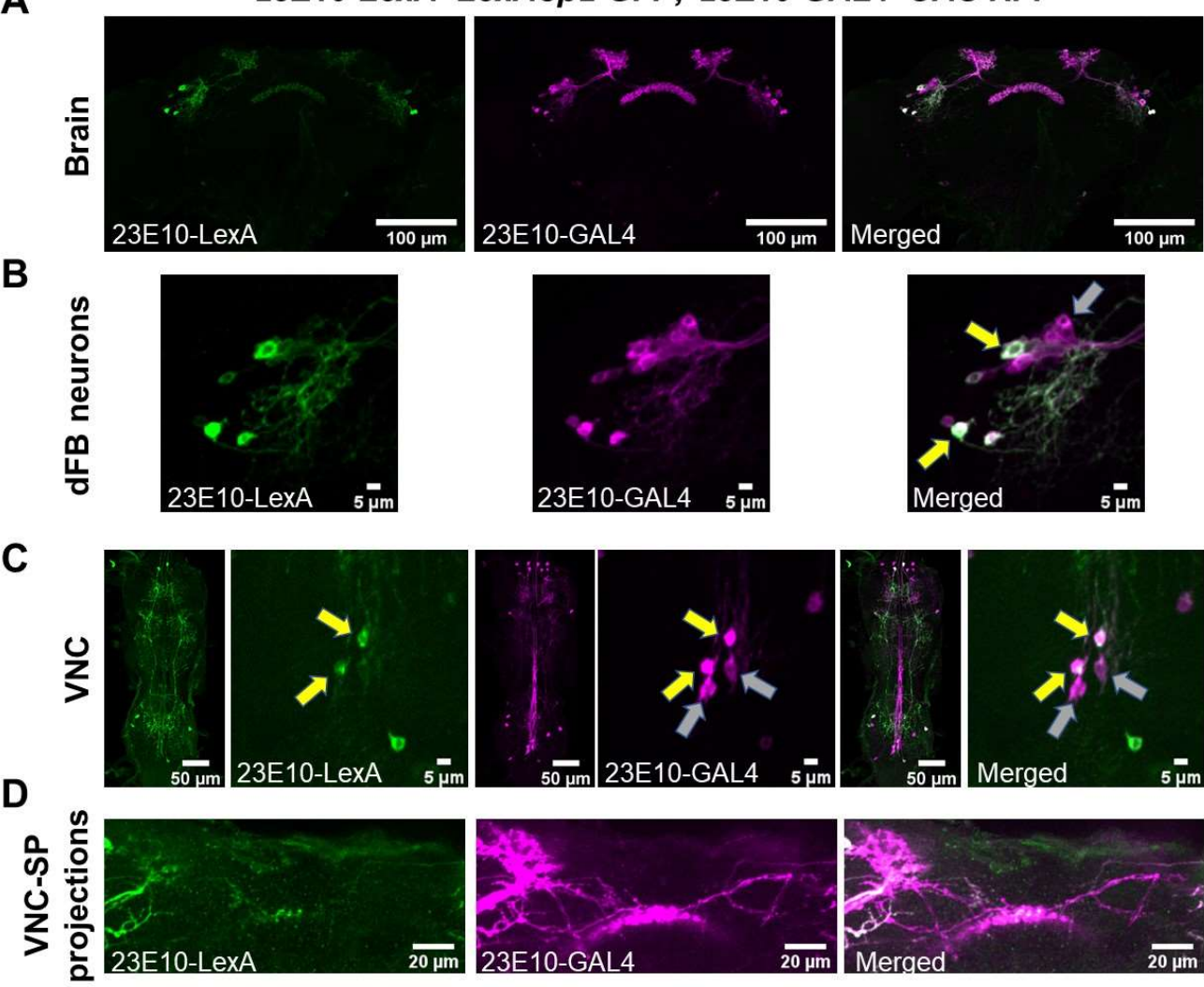

$\mathrm{E}$

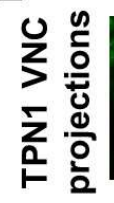

23E10-LexA
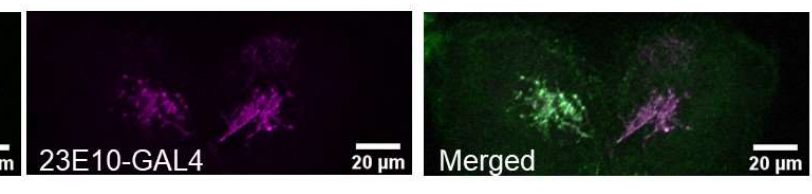

$\mathbf{F}$

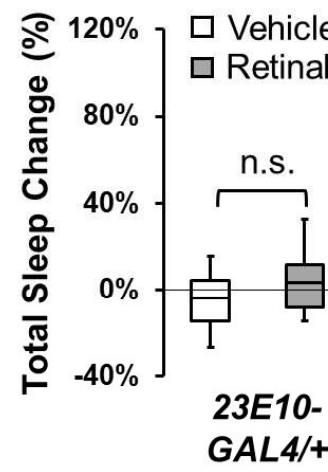

GAL4/+

UAS-

CsChrimson/+

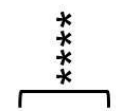<smiles>[Te]=C1CCCCC1</smiles>

23E10-

GAL4>UAS-

CsChrimson

G

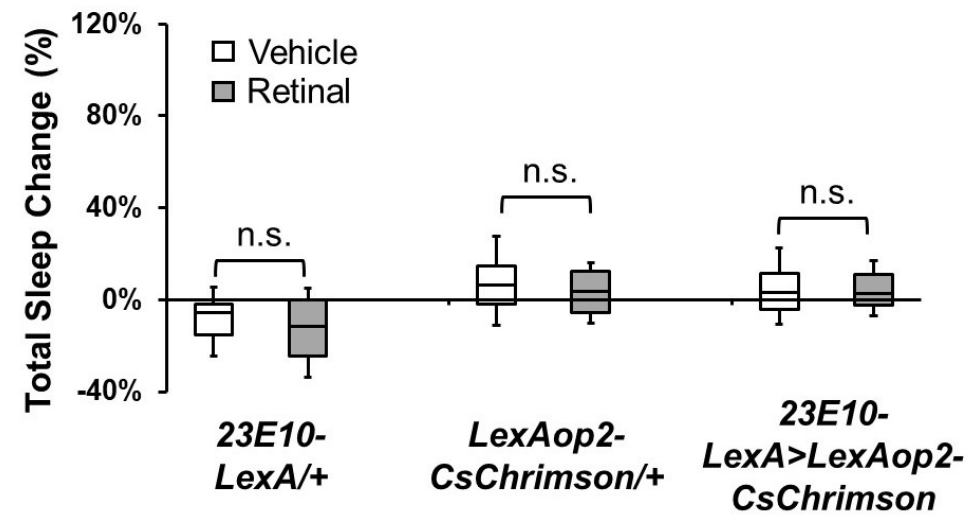


Figure 8

A

\section{E10-GAL4 neurons}

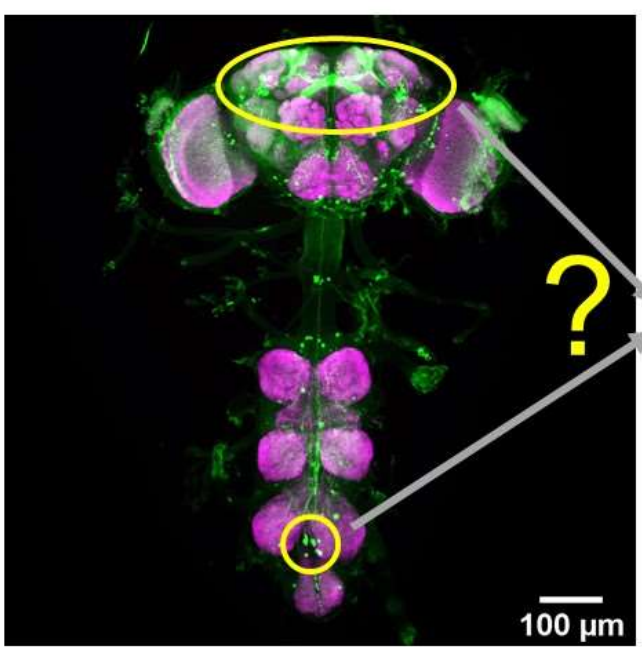

C

Silencing 23E10-GAL4 VNC-SP neurons

n.s.

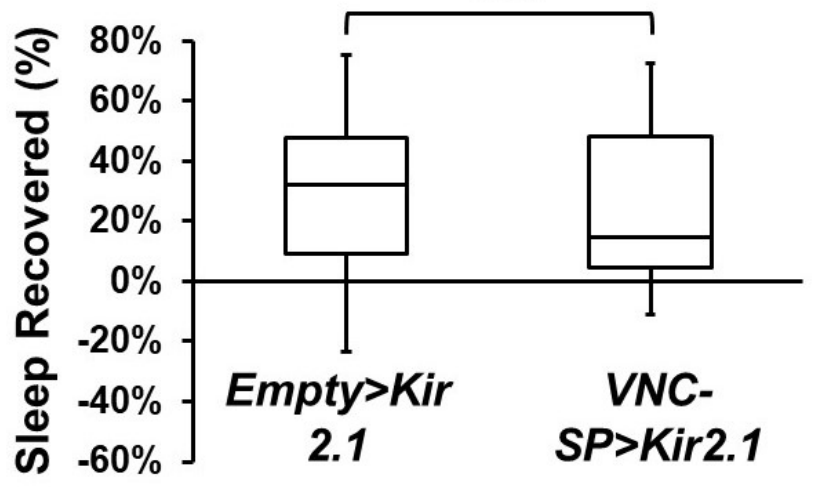

Sleep
B Homeostasis

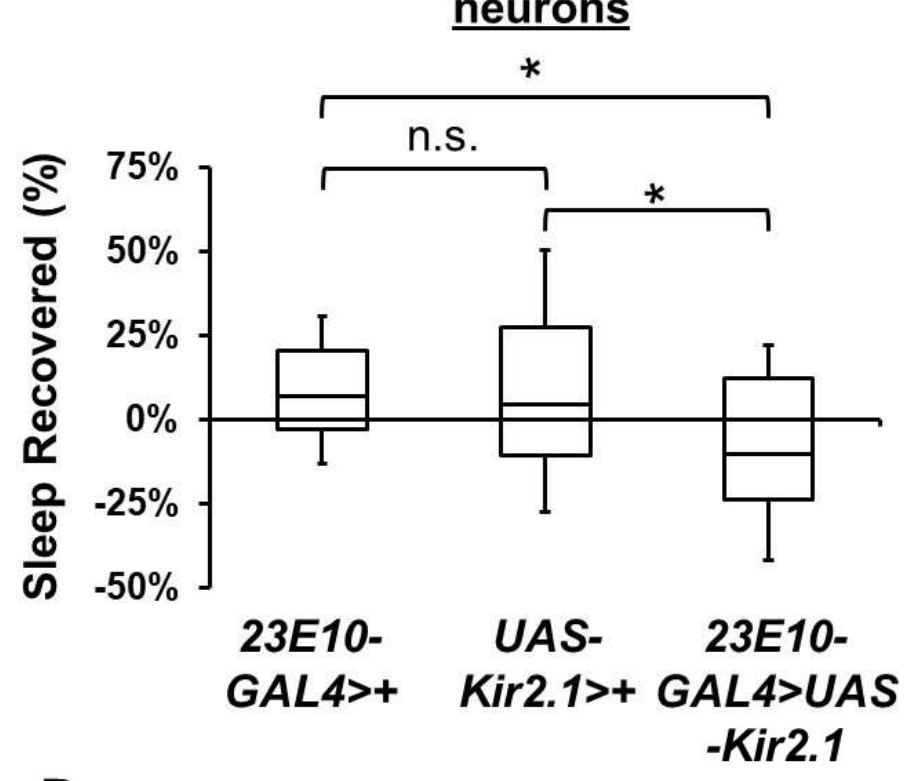

Silencing most 23E10GAL4 dFB neurons

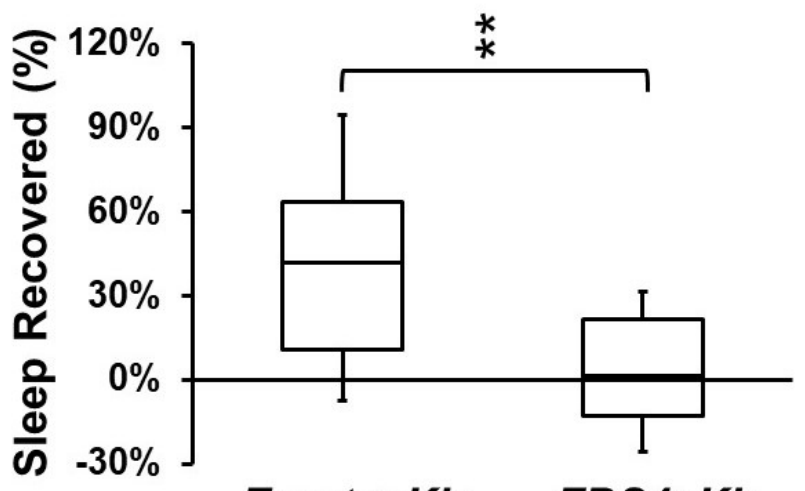

Empty>Kir

2.1

2.1 
Table 1

\begin{tabular}{|c|c|c|}
\hline & Average (n) & Range \\
\hline dFB neurons & $25.68 \pm 0.50(19)$ & $23-30$ \\
\hline $\begin{array}{c}\text { All brain } \\
\text { neurons }\end{array}$ & $51.79 \pm 1.05(19)$ & $44-62$ \\
\hline VNC neurons & $17.56 \pm 0.99(9)$ & $12-22$ \\
\hline
\end{tabular}


bioRxiv preprint doi: https://doi.org/10.1101/2022.02.02.478882; this version posted February 9,2022 . The copyright holder for this preprint

(which was not certified by peer review) is the author/funder, who has granted bioRxiv a license to display the preprint in perpetuity. It is made available under aCC-BY-NC-ND 4.0 International license.

\section{Table 2}

\begin{tabular}{|c|c|c|c|c|c|c|c|c|}
\hline $\begin{array}{l}\text { FBS } \\
\text { line }\end{array}$ & AD component & $\begin{array}{l}\text { Average whole } \\
\text { brain dFB } \\
\text { neurons } \pm \text { SEM } \\
\text { (n) }\end{array}$ & $\begin{array}{c}\text { Range } \\
\text { dFB } \\
\text { neurons }\end{array}$ & $\begin{array}{c}\text { Bowtie } \\
\text { (Y/N) }\end{array}$ & $\begin{array}{l}\text { Range of } \\
\text { additional } \\
\text { cells in } \\
\text { brain }\end{array}$ & $\begin{array}{l}\text { Average VNC } \\
\text { cells in } \\
\text { metathoracic } \\
\text { ganglion } \pm \\
\text { SEM }(\mathrm{n})\end{array}$ & $\begin{array}{l}\text { TPN1 } \\
\text { (Y/N) }\end{array}$ & $\begin{array}{c}\text { Range of } \\
\text { additional } \\
\text { cells in VNC }\end{array}$ \\
\hline FBS 1 & 12D12-AD & $4.64 \pm 0.40(n=14)$ & $2-7$ & $Y$ & 0 & $\begin{array}{l}2.00 \pm 0.32 \\
(n=11)\end{array}$ & $\mathrm{N}$ & 0 \\
\hline FBS 4 & 84C10-AD & $\begin{array}{l}20.80 \pm 0.35 \\
(n=15)\end{array}$ & $18-23$ & $\mathrm{~N}$ & 0 & $\begin{array}{l}0.00 \pm 0.50 \\
(n=15)\end{array}$ & $\mathrm{N}$ & 0 \\
\hline FBS 5 & 23E12-AD & $0 \pm 0(n=26)$ & 0 & $Y$ & $5-10$ & $\begin{array}{l}3.89 \pm 0.11 \\
(n=26)\end{array}$ & $Y$ & 0 \\
\hline FBS 6 & $42 \mathrm{H} 01-\mathrm{AD}$ & $3.85 \pm 0.52(n=13)$ & $1-4$ & $\mathrm{~N}$ & 0 & $\begin{array}{l}0.00 \pm 0.00 \\
(n=8)\end{array}$ & $\mathrm{N}$ & 0 \\
\hline $\begin{array}{l}\text { FBS } \\
25\end{array}$ & 22A12-AD & $0 \pm 0(n=5)$ & 0 & $\mathrm{~N}$ & 0 & $\begin{array}{l}0.00 \pm 0.00 \\
(n=5)\end{array}$ & $\mathrm{N}$ & 0 \\
\hline $\begin{array}{l}\text { FBS } \\
28\end{array}$ & 54G07-AD & $\begin{array}{l}13.08 \pm 0.63 \\
(n=12)\end{array}$ & $10-18$ & $\bar{Y}$ & $0-1$ & $\begin{array}{l}3.09 \pm 0.17 \\
(n=22)\end{array}$ & $\mathrm{N}$ & 0 \\
\hline $\begin{array}{l}\text { FBS } \\
33\end{array}$ & $49 \mathrm{CO} 4-\mathrm{AD}$ & $\begin{array}{l}26.50 \pm 1.15 \\
(n=10)\end{array}$ & $23-31$ & $Y$ & 0 & $\begin{array}{l}3.17 \pm 0.32 \\
(n=12)\end{array}$ & $Y$ & 0 \\
\hline $\begin{array}{l}\text { FBS } \\
35\end{array}$ & $65 C 03-A D$ & $5.00 \pm 1.06(n=6)$ & $2-8$ & $Y$ & $1-2$ & $\begin{array}{l}3.60 \pm 0.24 \\
(n=5)\end{array}$ & $Y$ & 0 \\
\hline $\begin{array}{l}\text { FBS } \\
41\end{array}$ & VT058545-AD & $\begin{array}{l}11.50 \pm 0.43 \\
(n=10)\end{array}$ & $10-14$ & $\mathrm{~N}$ & $0-2$ & $\begin{array}{l}3.00 \pm 0.41 \\
(n=4)\end{array}$ & $Y$ & 0 \\
\hline $\begin{array}{l}\text { FBS } \\
42\end{array}$ & 12G09-AD & $21.56 \pm 0.80(=10)$ & $18-25$ & $Y$ & $2-4$ & $\begin{array}{l}3.00 \pm 0.37 \\
(n=6)\end{array}$ & $Y$ & $0-2$ \\
\hline $\begin{array}{l}\text { FBS } \\
45\end{array}$ & VT050238-AD & $25.11 \pm 1.41(n=9)$ & $21-32$ & $Y$ & 0 & $\begin{array}{l}2.40 \pm 0.16 \\
(n=15)\end{array}$ & $\mathrm{N}$ & 0 \\
\hline $\begin{array}{l}\text { FBS } \\
53\end{array}$ & VT043400-AD & $23.86 \pm 0.51(n=7)$ & $22-26$ & $Y$ & 0 & $\begin{array}{l}2.91 \pm 0.31 \\
(n=11)\end{array}$ & $\mathrm{N}$ & $0-1$ \\
\hline $\begin{array}{l}\text { FBS } \\
57\end{array}$ & VT025720-AD & $7.40 \pm 0.68(n=5)$ & $5-9$ & $Y$ & 0 & $\begin{array}{l}3.00 \pm 0.31 \\
(n=7)\end{array}$ & $Y$ & 0 \\
\hline $\begin{array}{l}\text { FBS } \\
58\end{array}$ & VT064566-AD & $21.50 \pm 1.28(n=6)$ & $18-27$ & $\mathrm{Y}$ & $0-2$ & $\begin{array}{l}3.10 \pm 0.28 \\
(n=10)\end{array}$ & $Y$ & $0-1$ \\
\hline $\begin{array}{l}\text { FBS } \\
60\end{array}$ & VT023818-AD & $24.56 \pm 0.50(n=9)$ & $22-27$ & $Y$ & 0 & $\begin{array}{l}3.27 \pm 0.38 \\
(n=11)\end{array}$ & $Y$ & $0-2$ \\
\hline $\begin{array}{l}\text { FBS } \\
64\end{array}$ & VT027956-AD & $15.00 \pm 2.12(n=7)$ & $11-19$ & $Y$ & 0 & $\begin{array}{l}3.40 \pm 0.49 \\
(n=10)\end{array}$ & $Y$ & 0 \\
\hline $\begin{array}{l}\text { FBS } \\
68\end{array}$ & VT023823-AD & $4.45 \pm 0.34(n=11)$ & $3-6$ & $Y$ & 0 & $\begin{array}{l}4.08 \pm 0.49 \\
(n=13)\end{array}$ & $Y$ & $0-4$ \\
\hline $\begin{array}{l}\text { FBS } \\
70\end{array}$ & VT034811-AD & $4.86 \pm 0.40(n=7)$ & $3-6$ & $Y$ & $0-7$ & $\begin{array}{l}3.90 \pm 0.38 \\
(n=10)\end{array}$ & $Y$ & $0-4$ \\
\hline $\begin{array}{l}\text { FBS } \\
72\end{array}$ & VT026953-AD & $24.40 \pm 1.50(n=7)$ & $20-28$ & $Y$ & $0-1$ & $\begin{array}{l}3.27 \pm 0.25(n= \\
10)\end{array}$ & $Y$ & 0 \\
\hline $\begin{array}{l}\text { FBS } \\
81\end{array}$ & 74G10-AD & $22.17 \pm 0.70(n=6)$ & $19-24$ & $Y$ & 0 & $\begin{array}{l}2.80 \pm 0.58 \\
(n=5)\end{array}$ & $\mathrm{N}$ & $0-2$ \\
\hline $\begin{array}{l}\text { FBS } \\
84\end{array}$ & VT007754-AD & $23.80 \pm 0.58(n=8)$ & $22-25$ & $\bar{Y}$ & $0-2$ & $\begin{array}{l}3.36 \pm 0.31 \\
(n=12)\end{array}$ & $\bar{Y}$ & $0-4$ \\
\hline $\begin{array}{l}\text { FBS } \\
87\end{array}$ & VT045793-AD & $\begin{array}{l}18.55 \pm 0.53 \\
(n=13)\end{array}$ & $17-22$ & $Y$ & $0-2$ & $\begin{array}{l}3.62 \pm 0.29 \\
(n=13)\end{array}$ & $Y$ & $0-6$ \\
\hline
\end{tabular}




\section{Figure 2-S1}
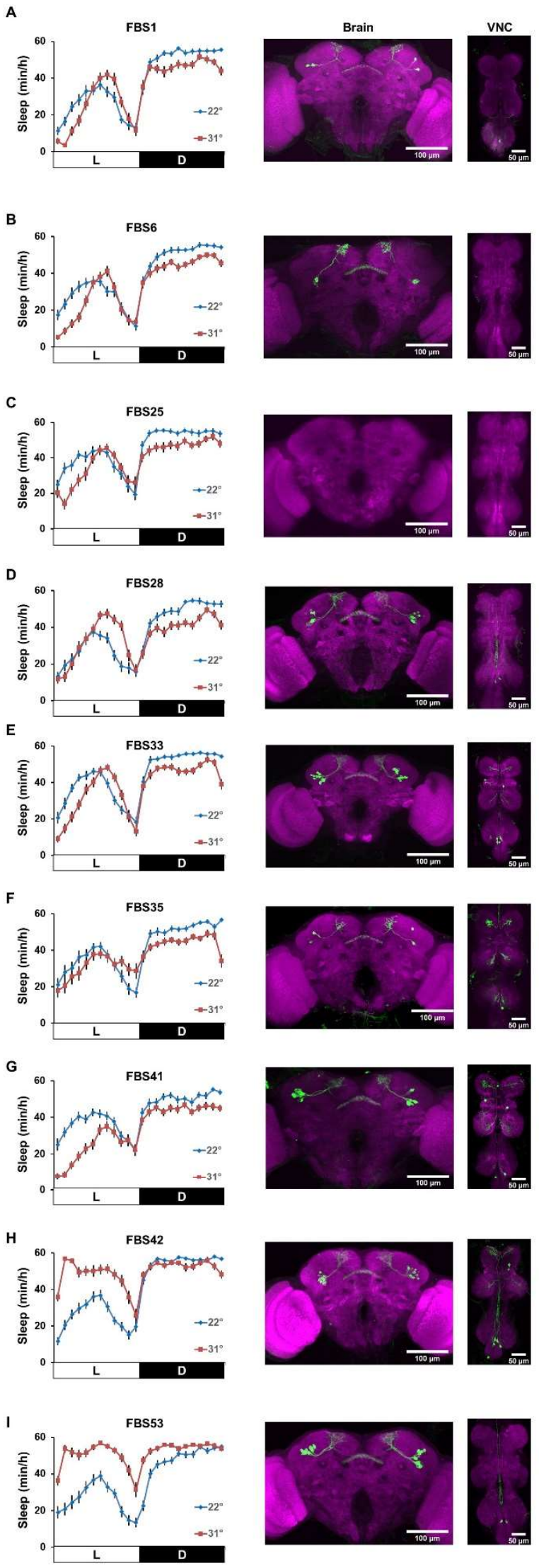
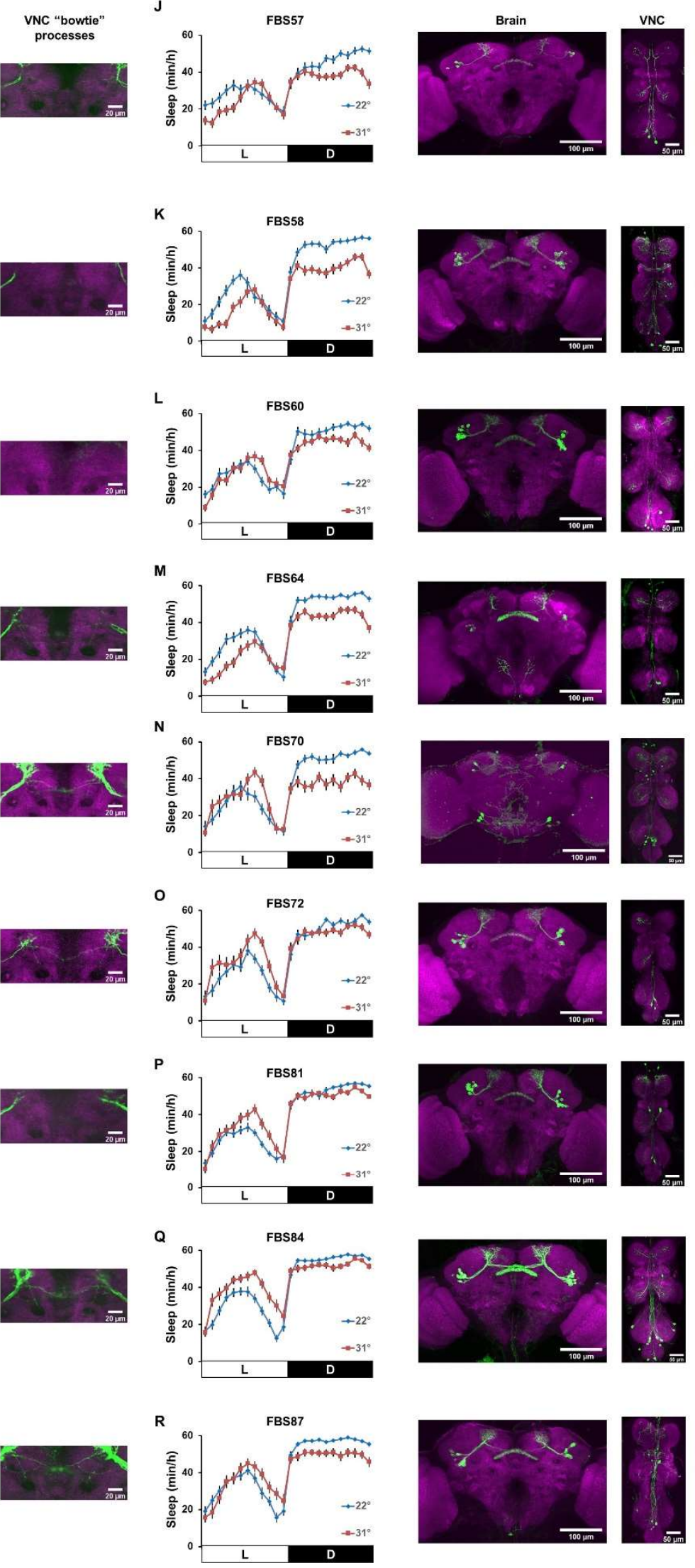
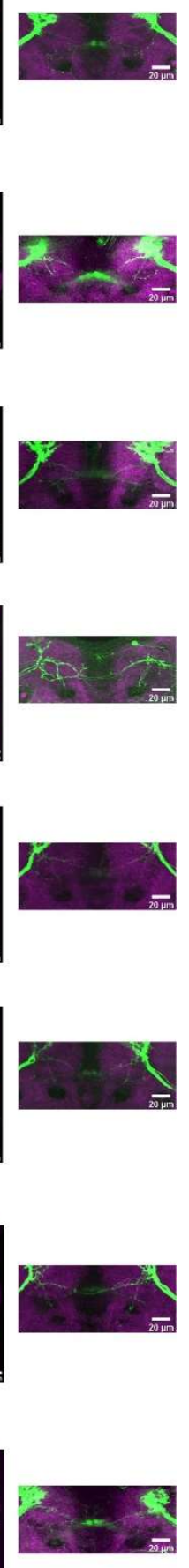
Figure 2-S2

A

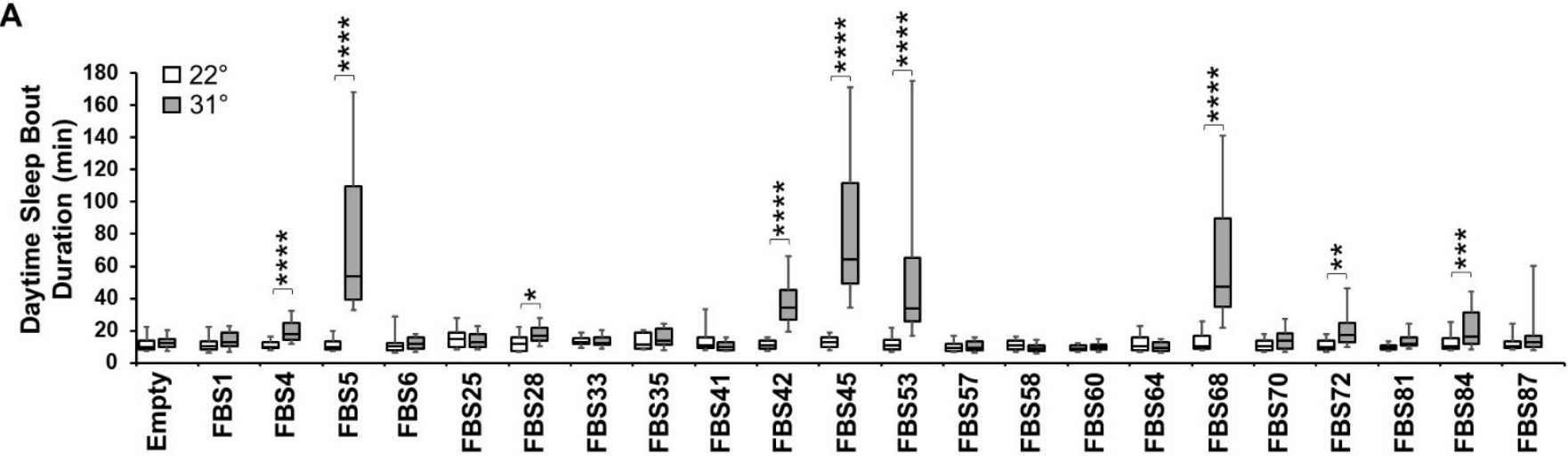

B

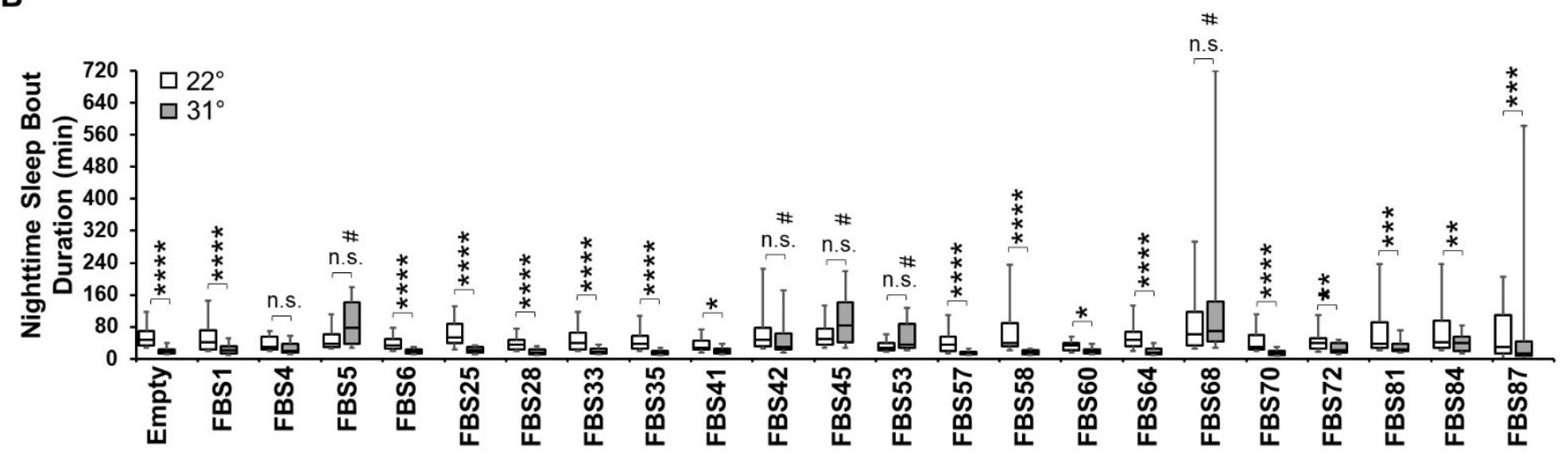


Figure 2-S3

A

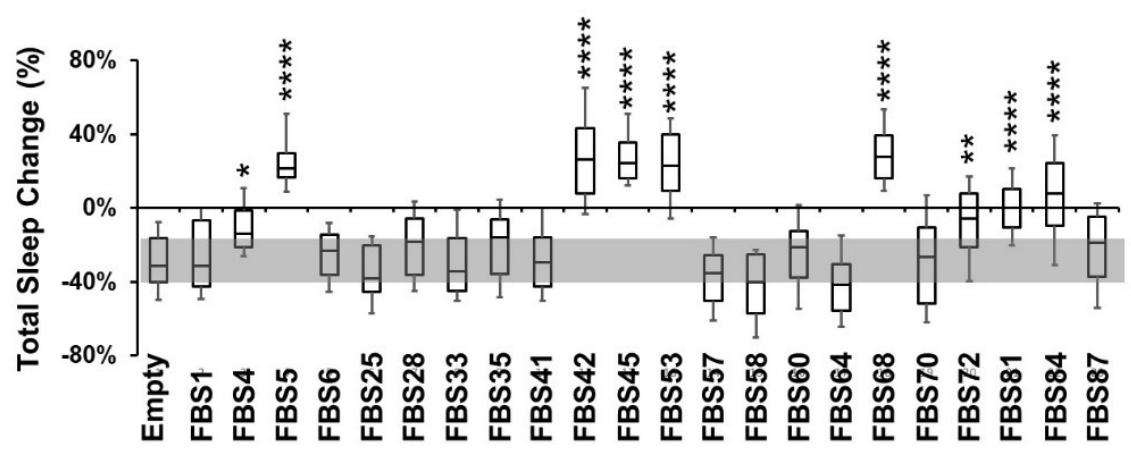

B

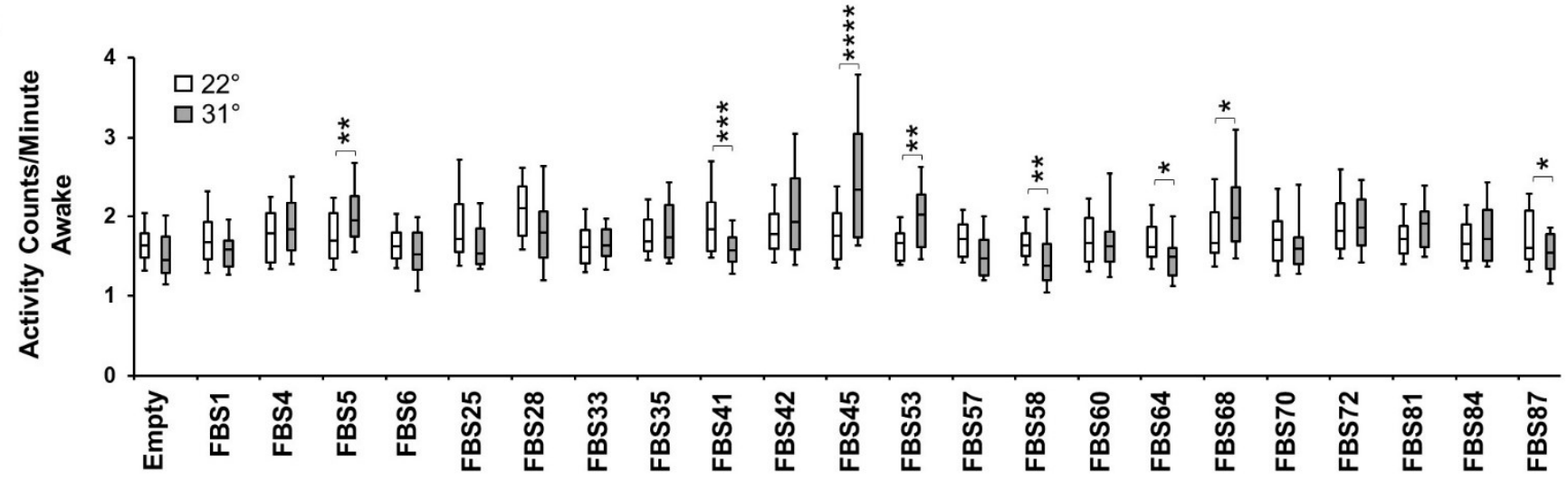

C
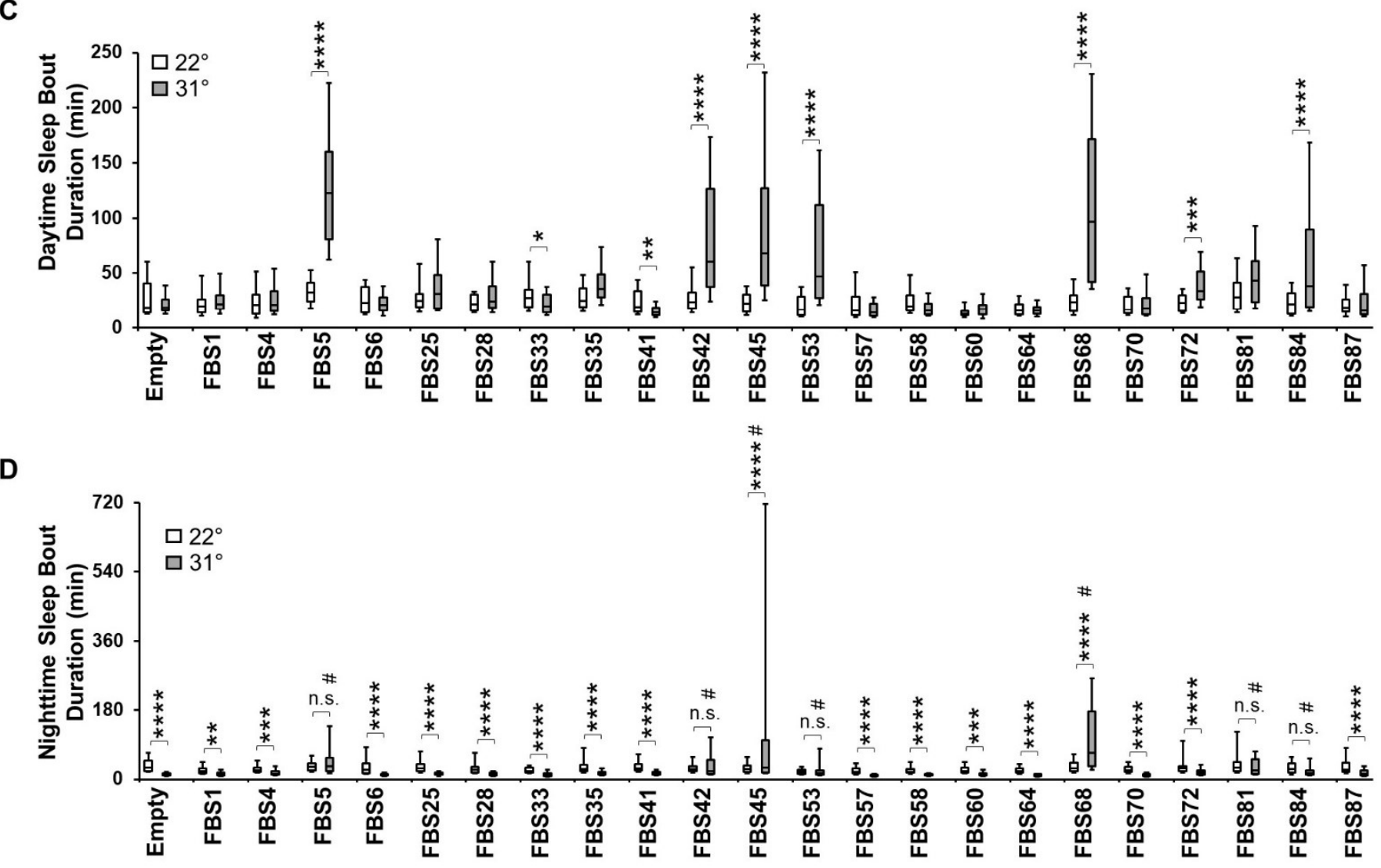
Figure 3-S1

A

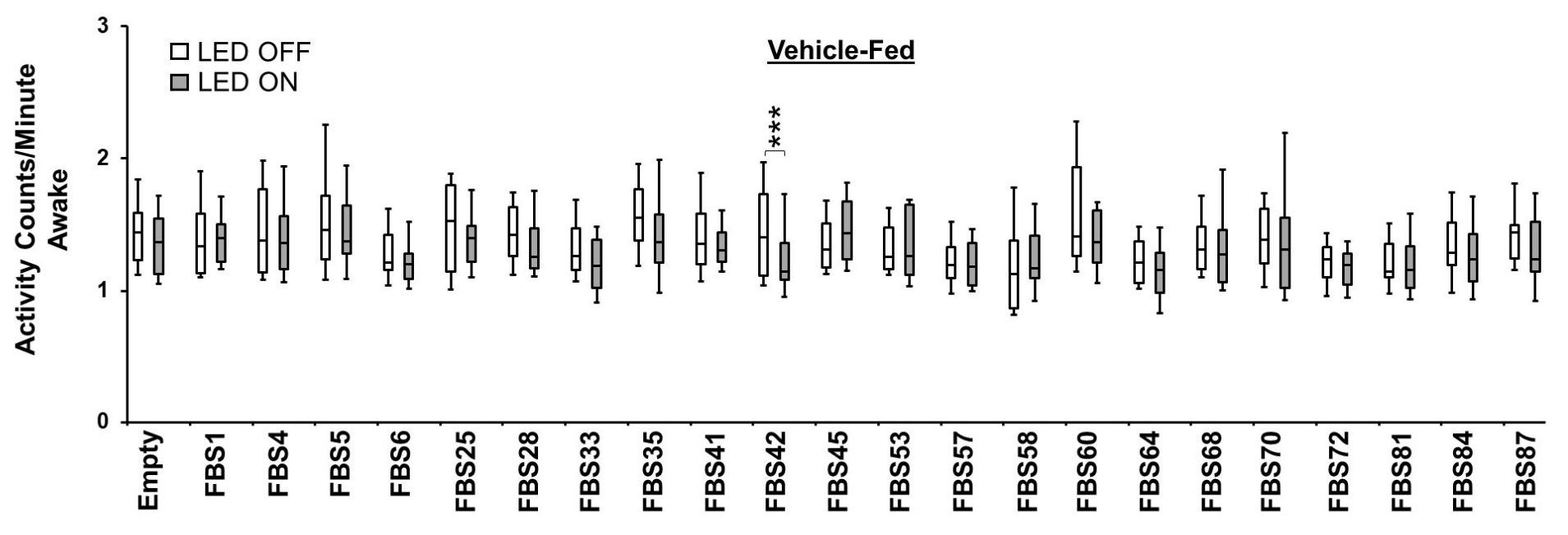

B
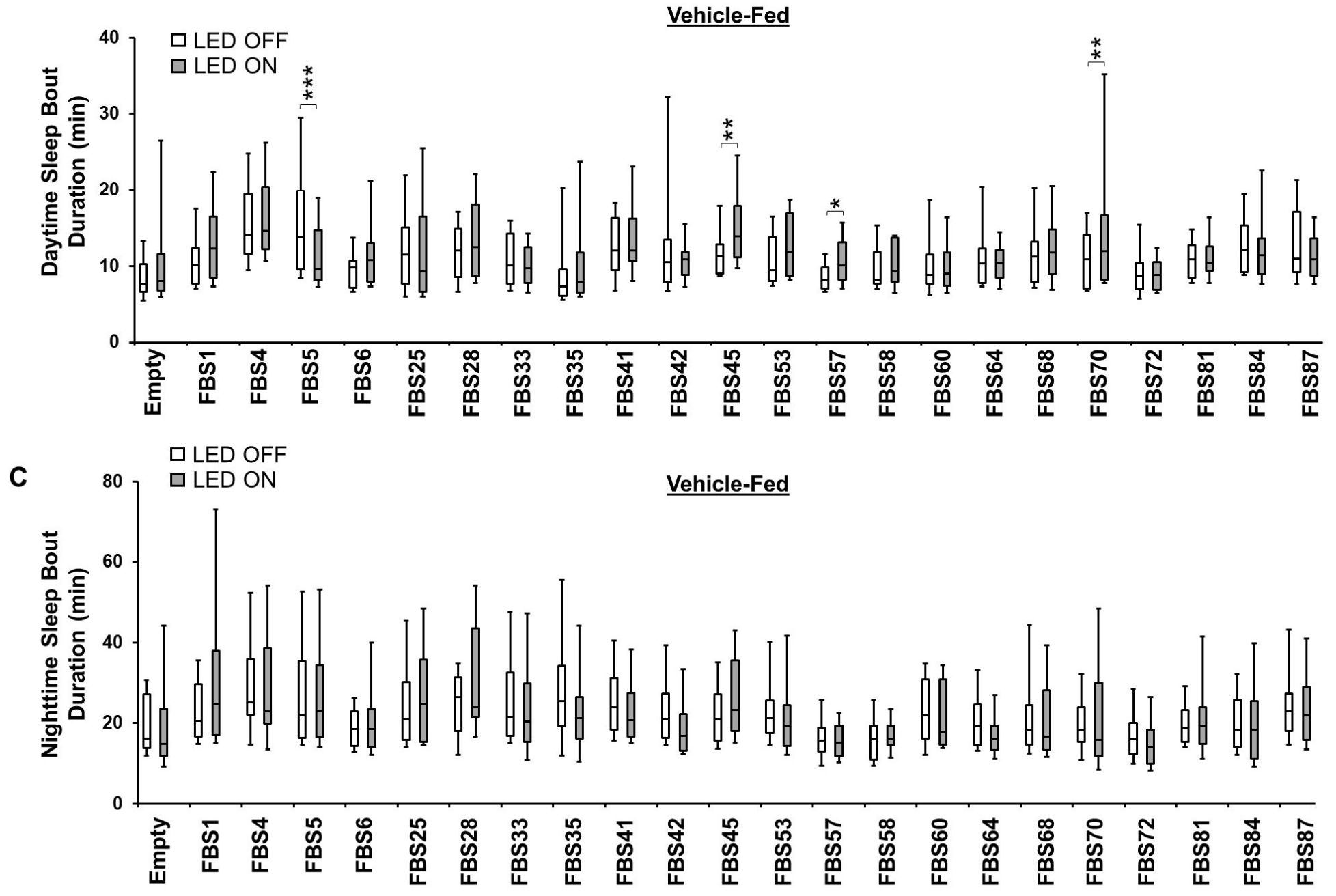


\section{Figure 3-S2}

A

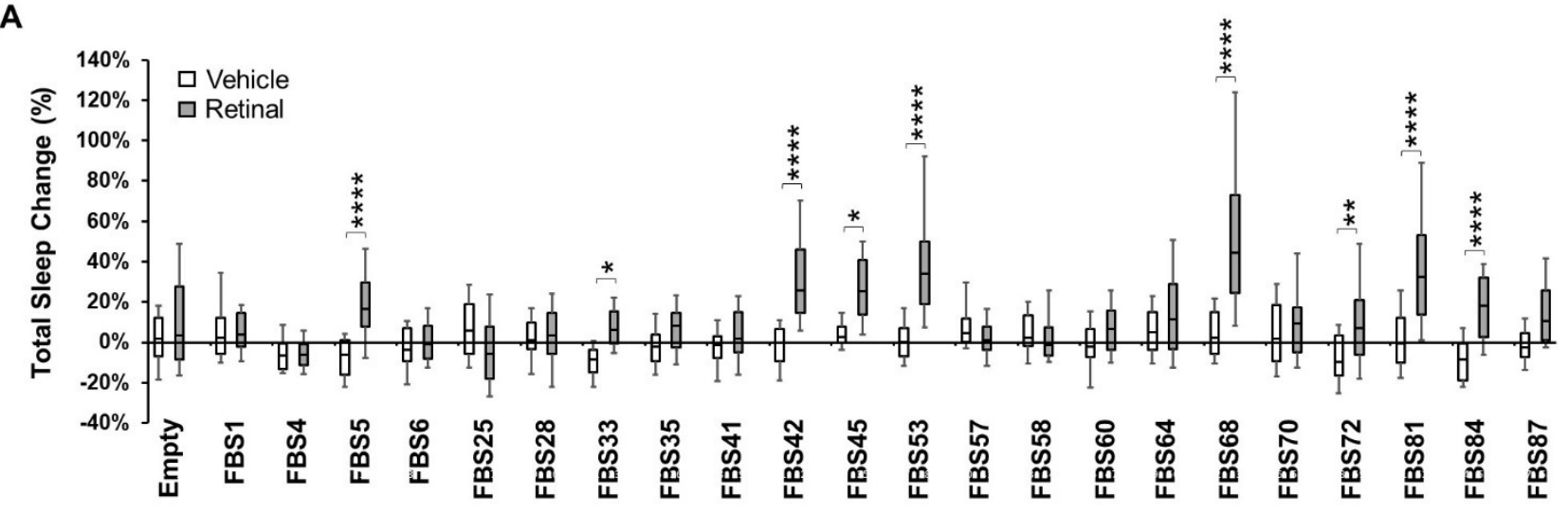

B

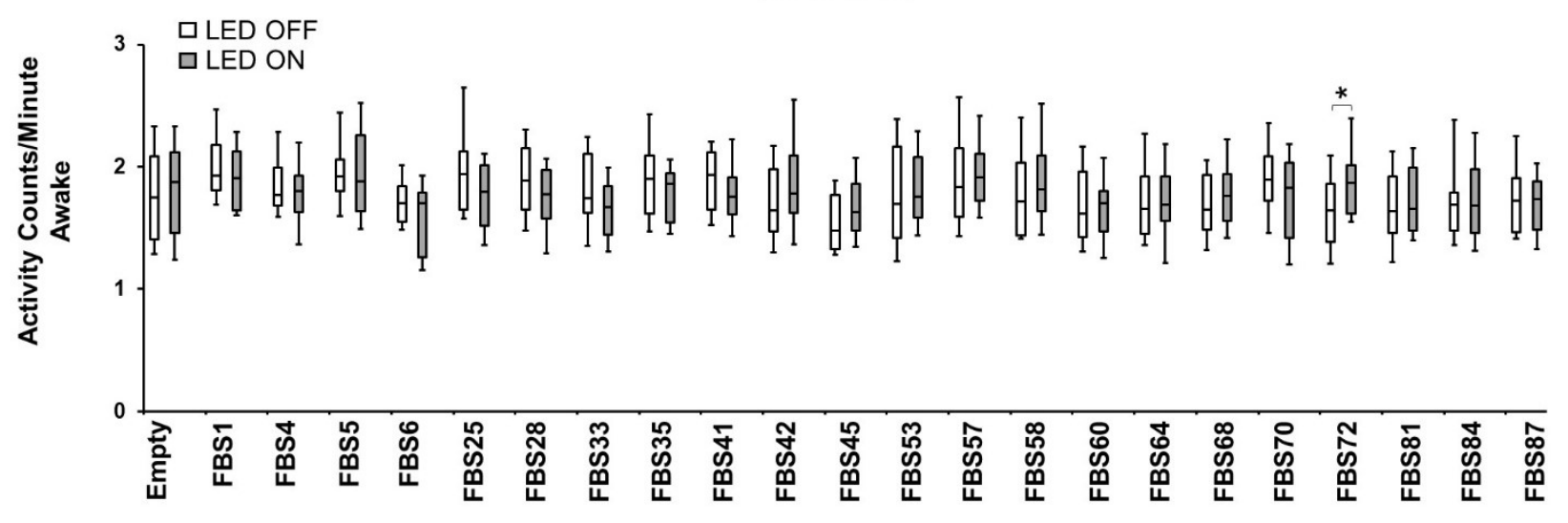

C

\section{Retinal-Fed}

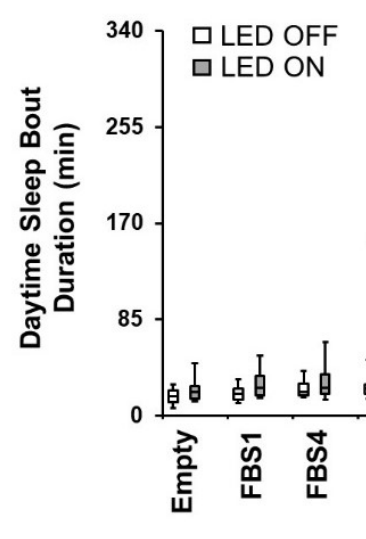

D

\section{Retinal-Fed}

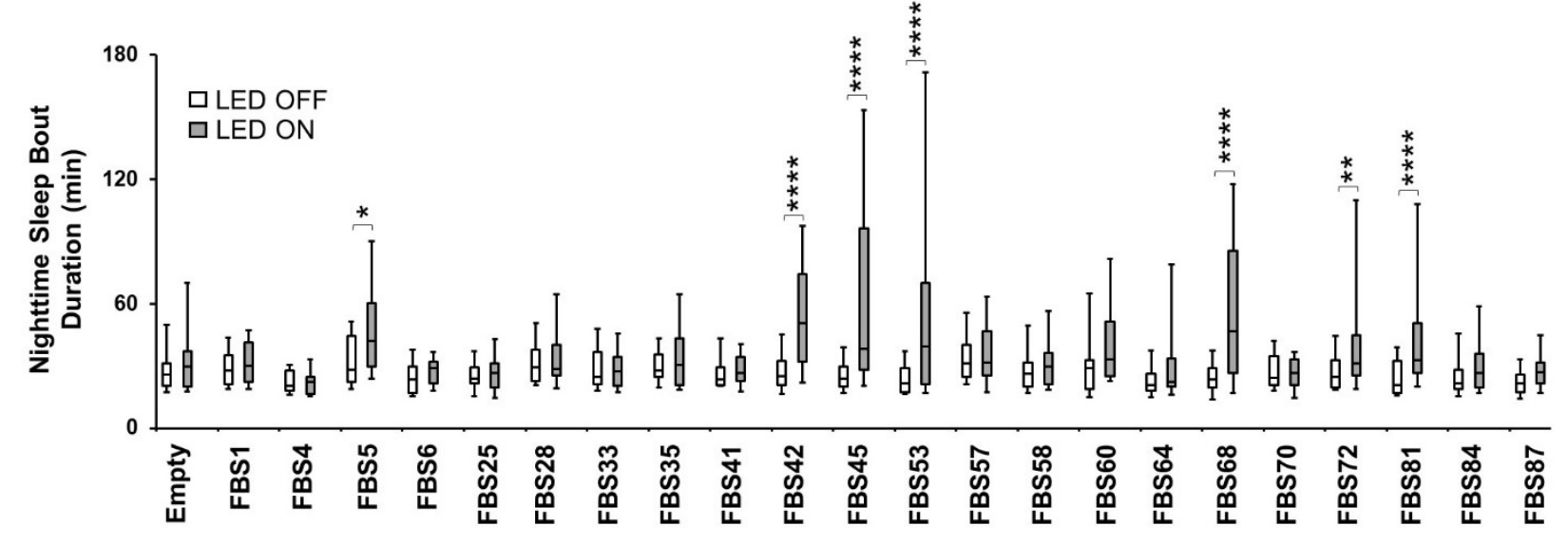




\section{Figure 3-S3}

A

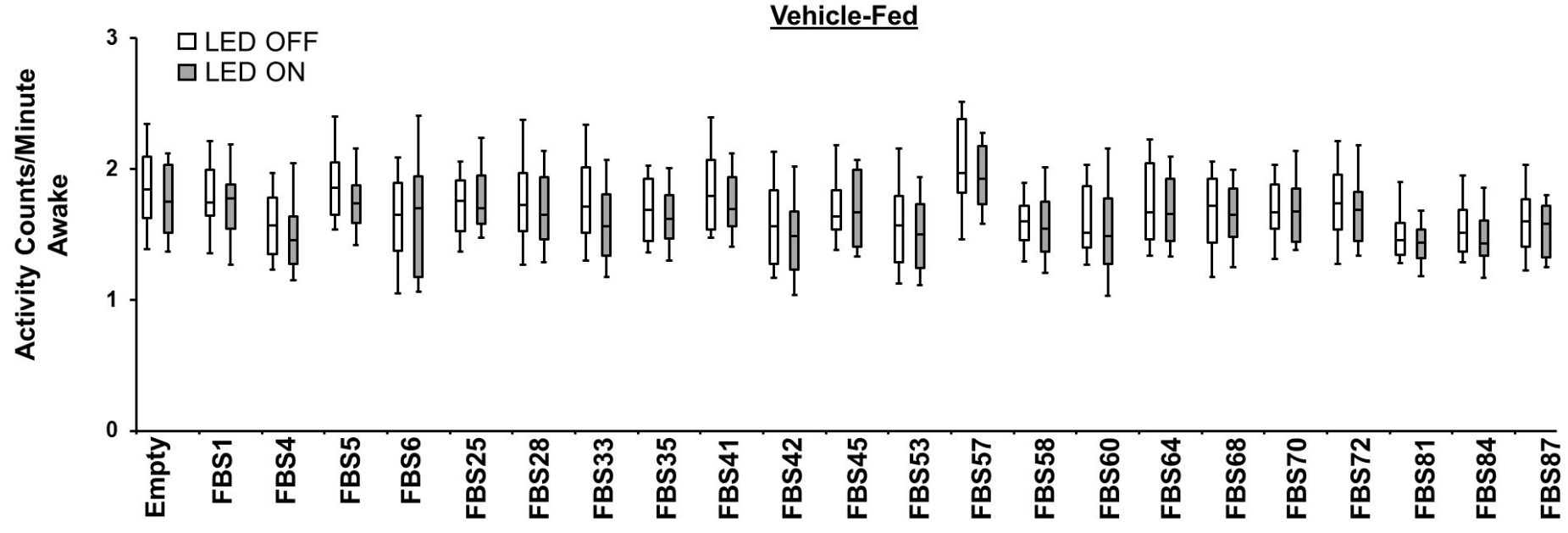

B

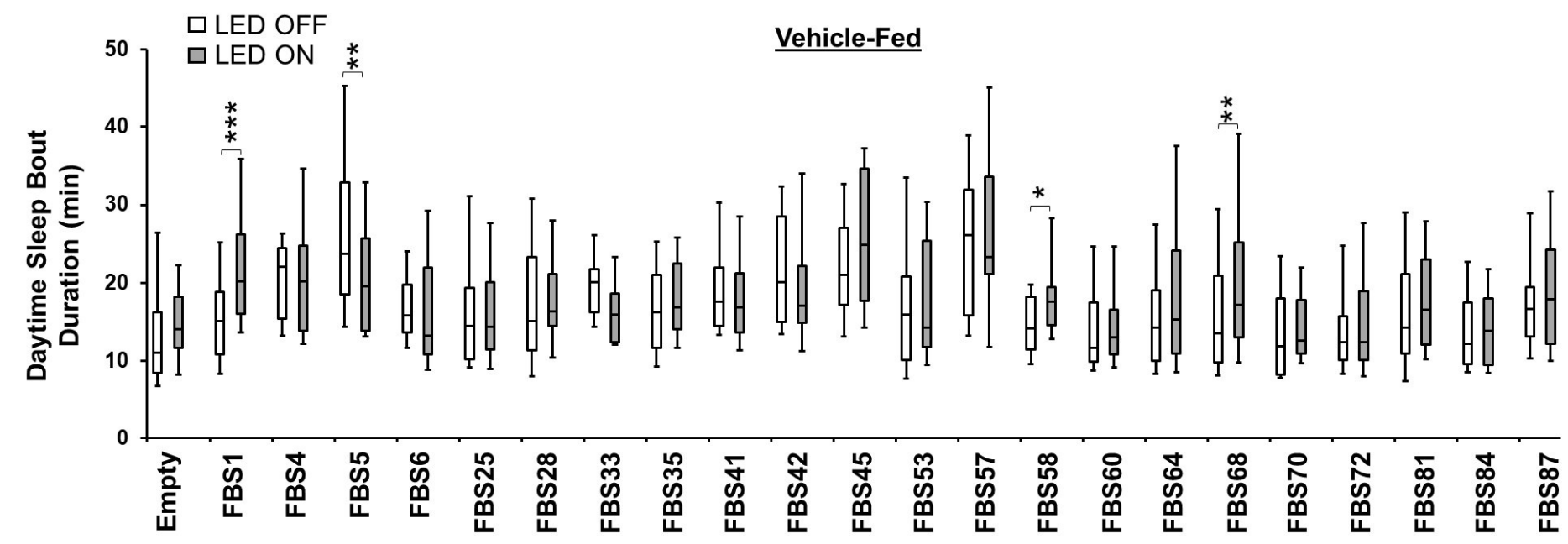

C

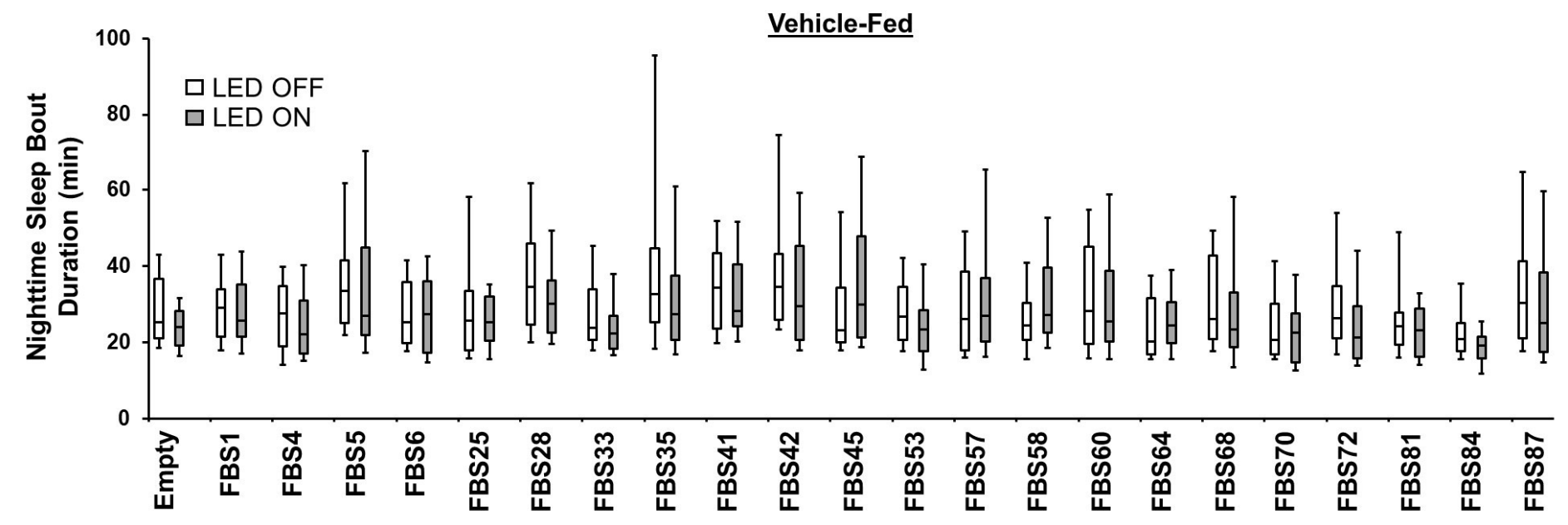




\section{Figure 4-S1}

A

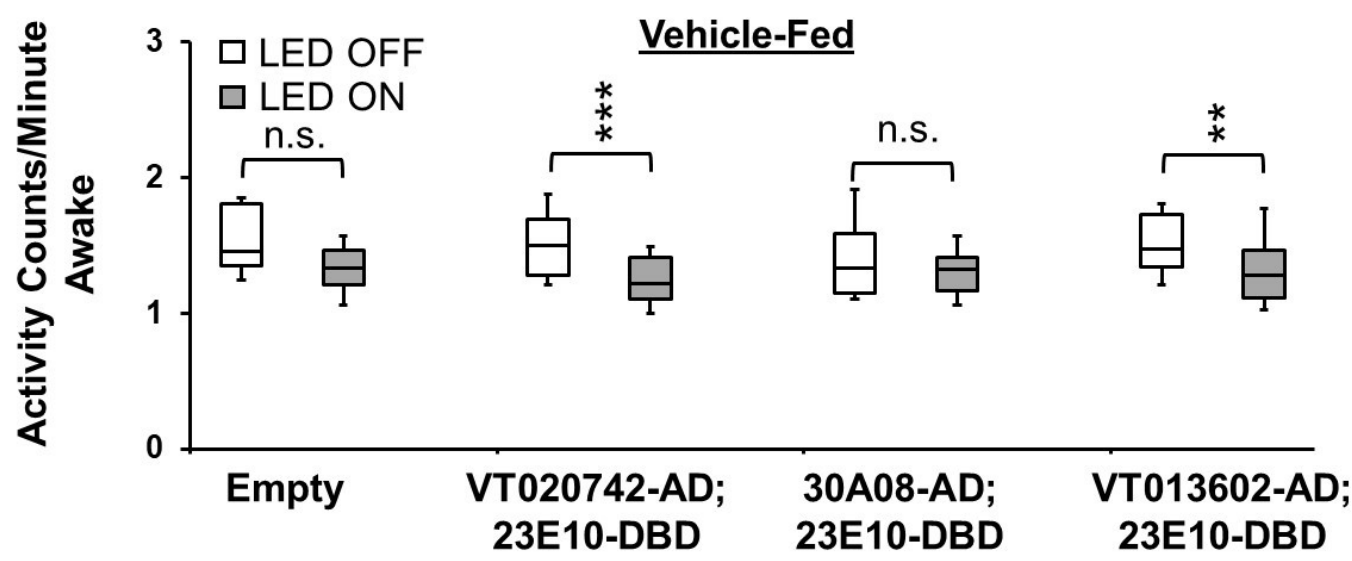

B

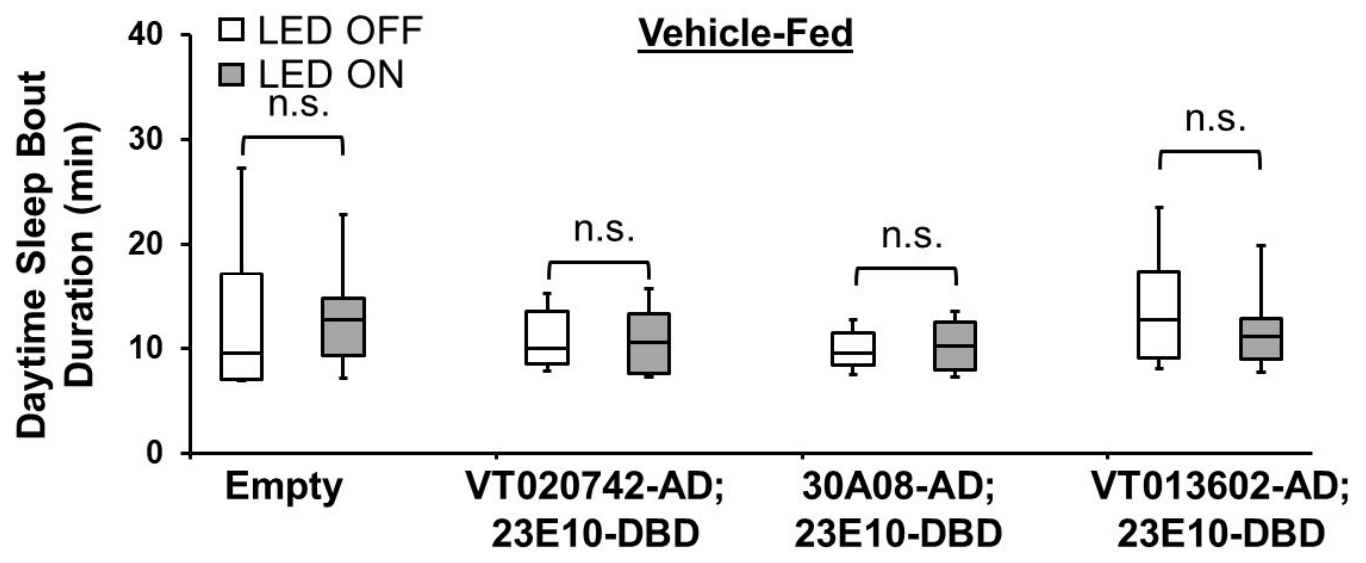

C

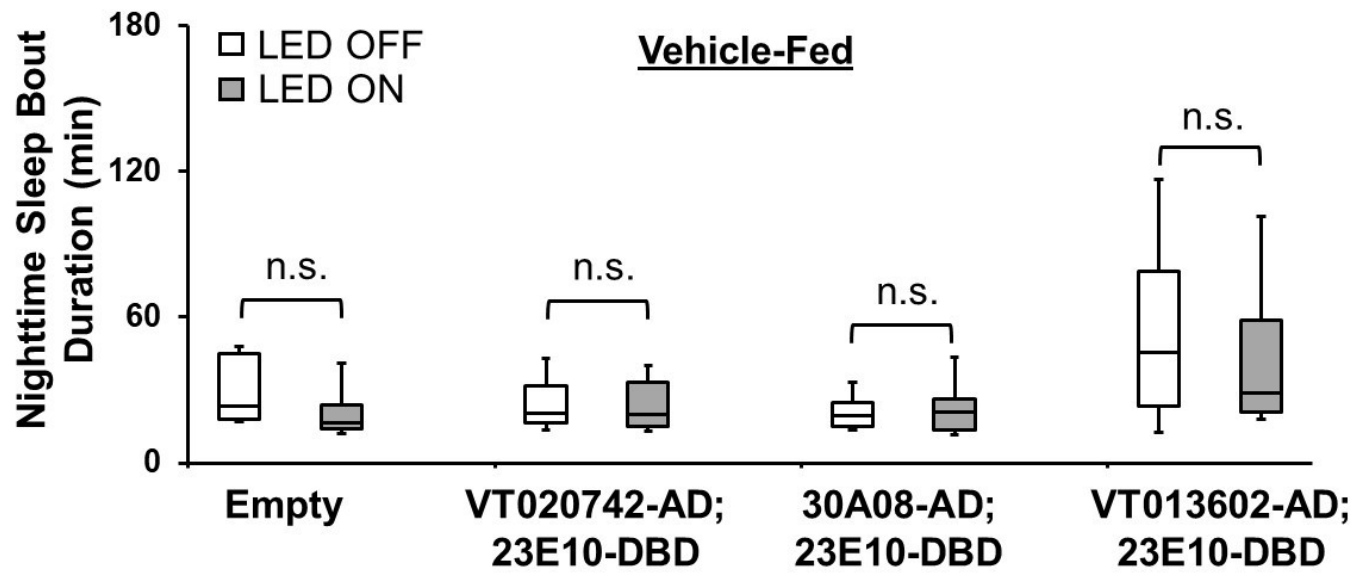




\section{Figure 4-S2}

A

\section{CsChrimson}

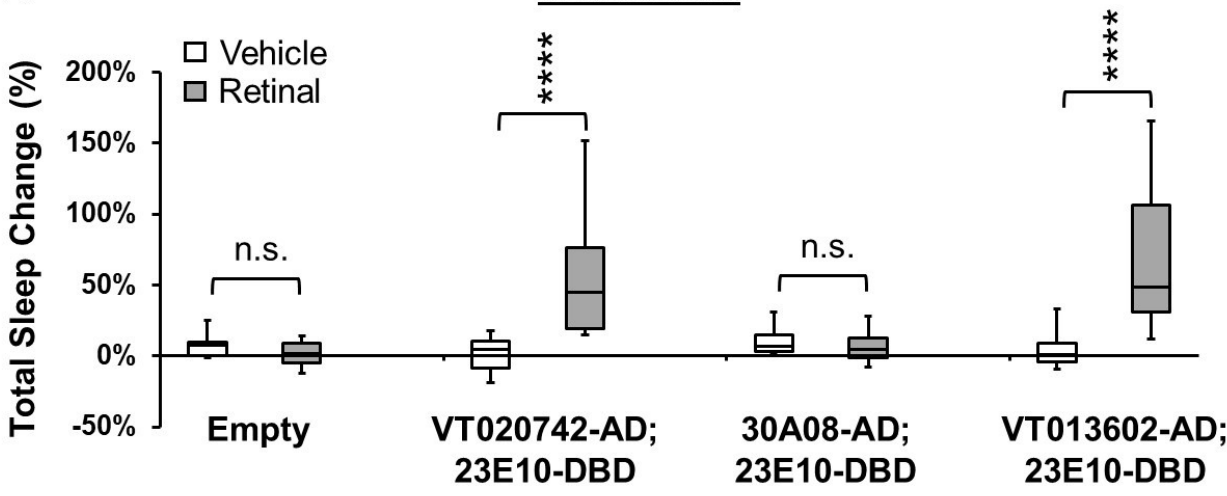

B

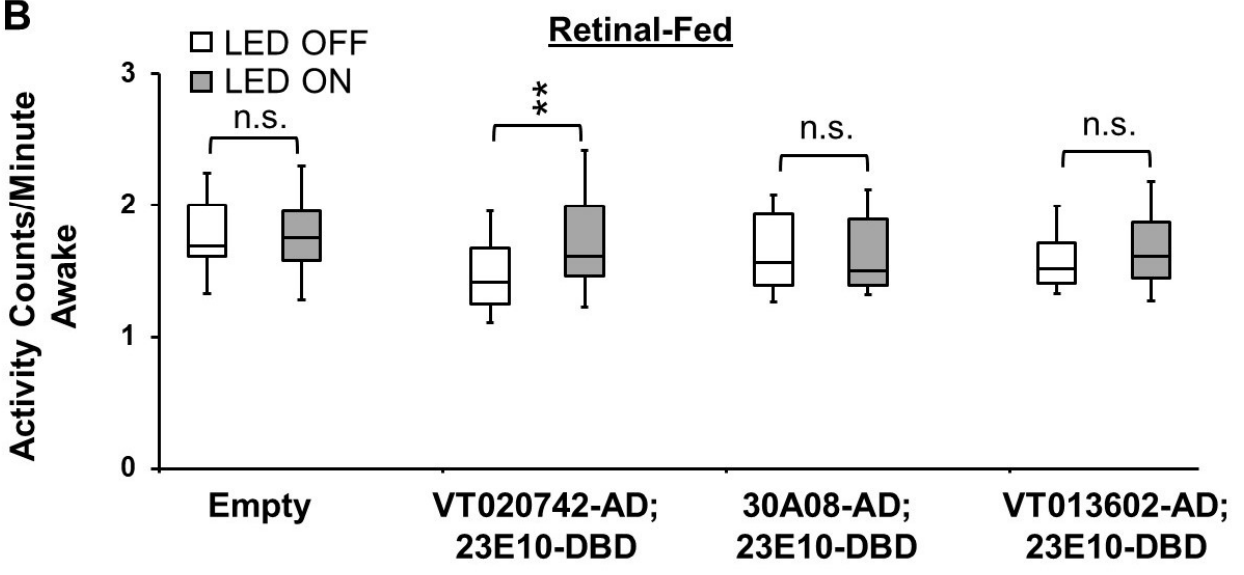

C

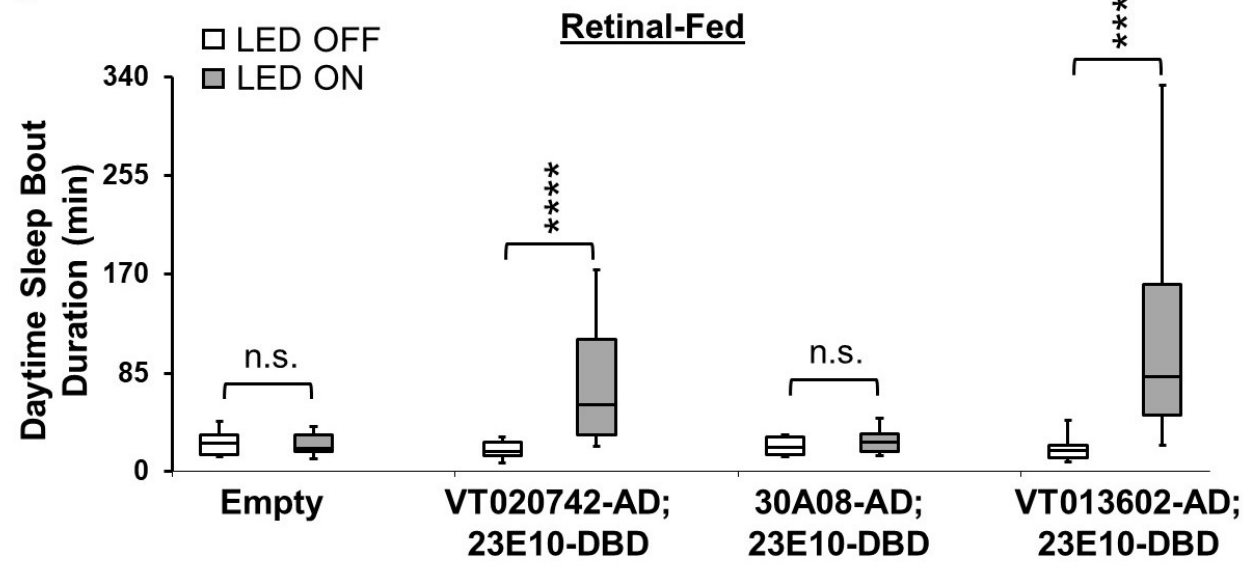

D

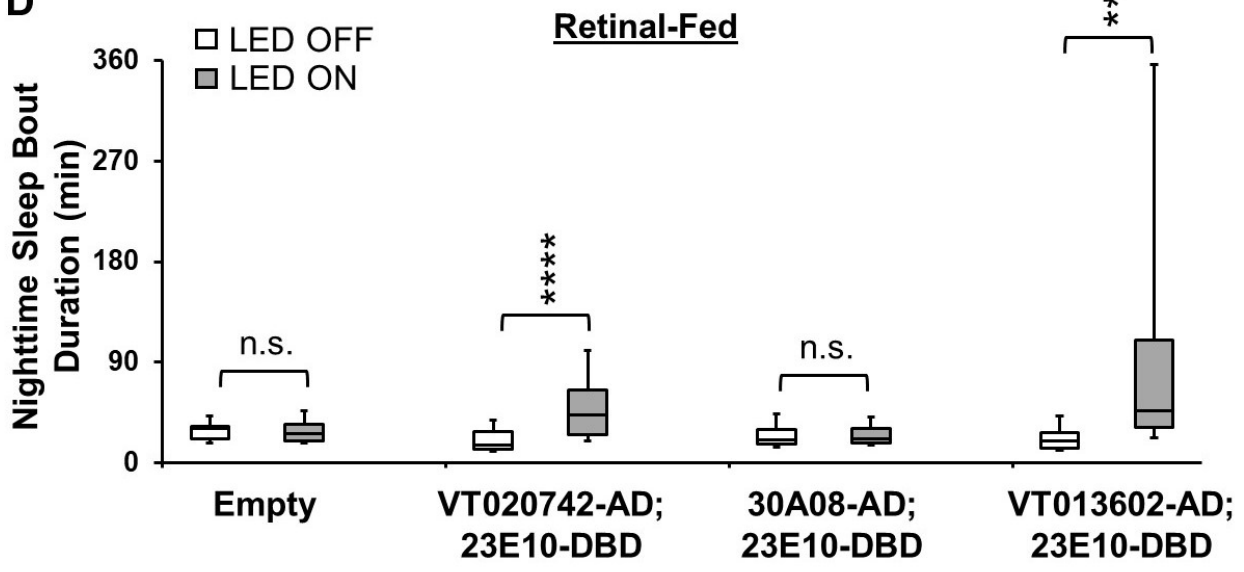


Figure 4-S3
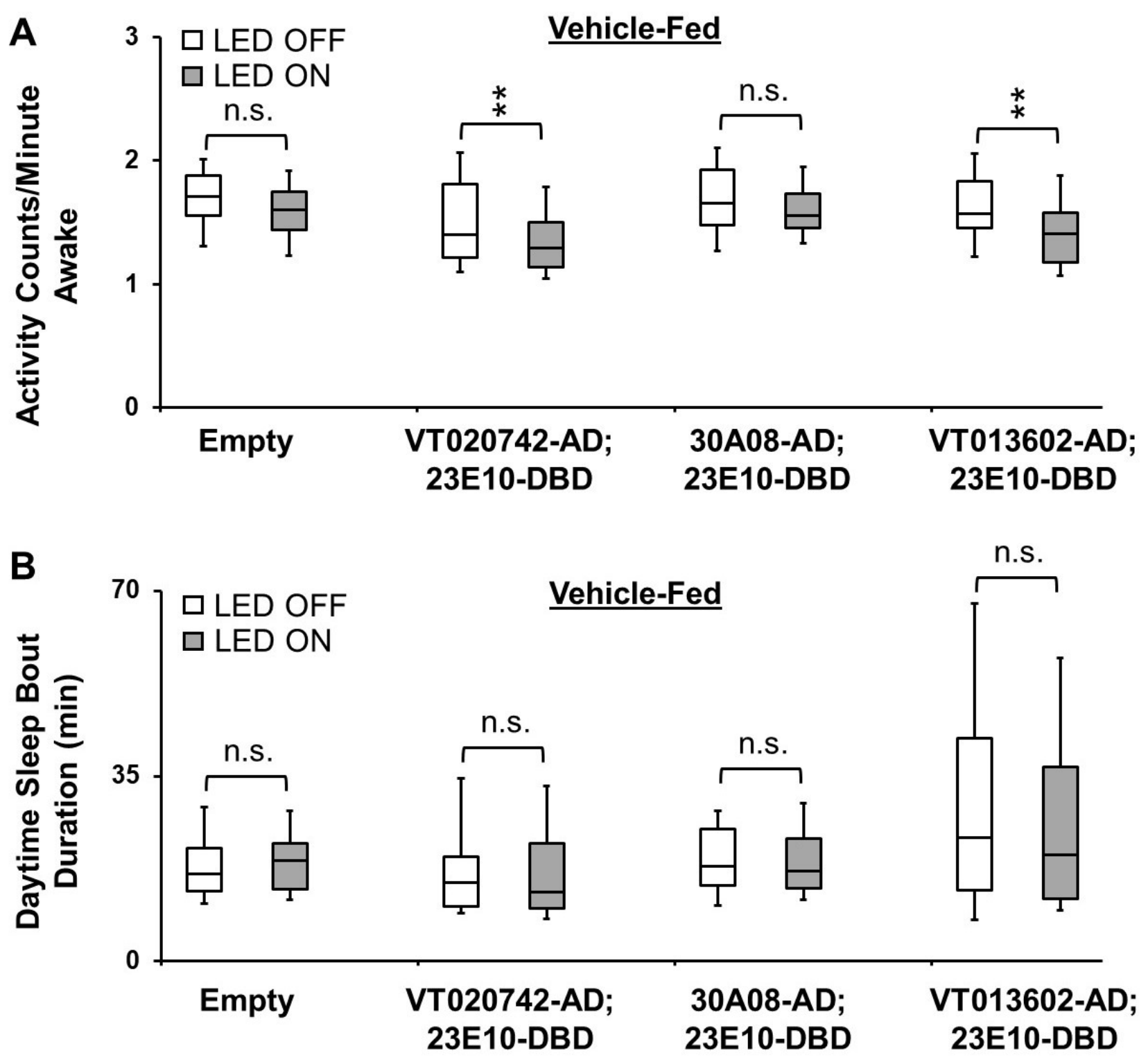

C

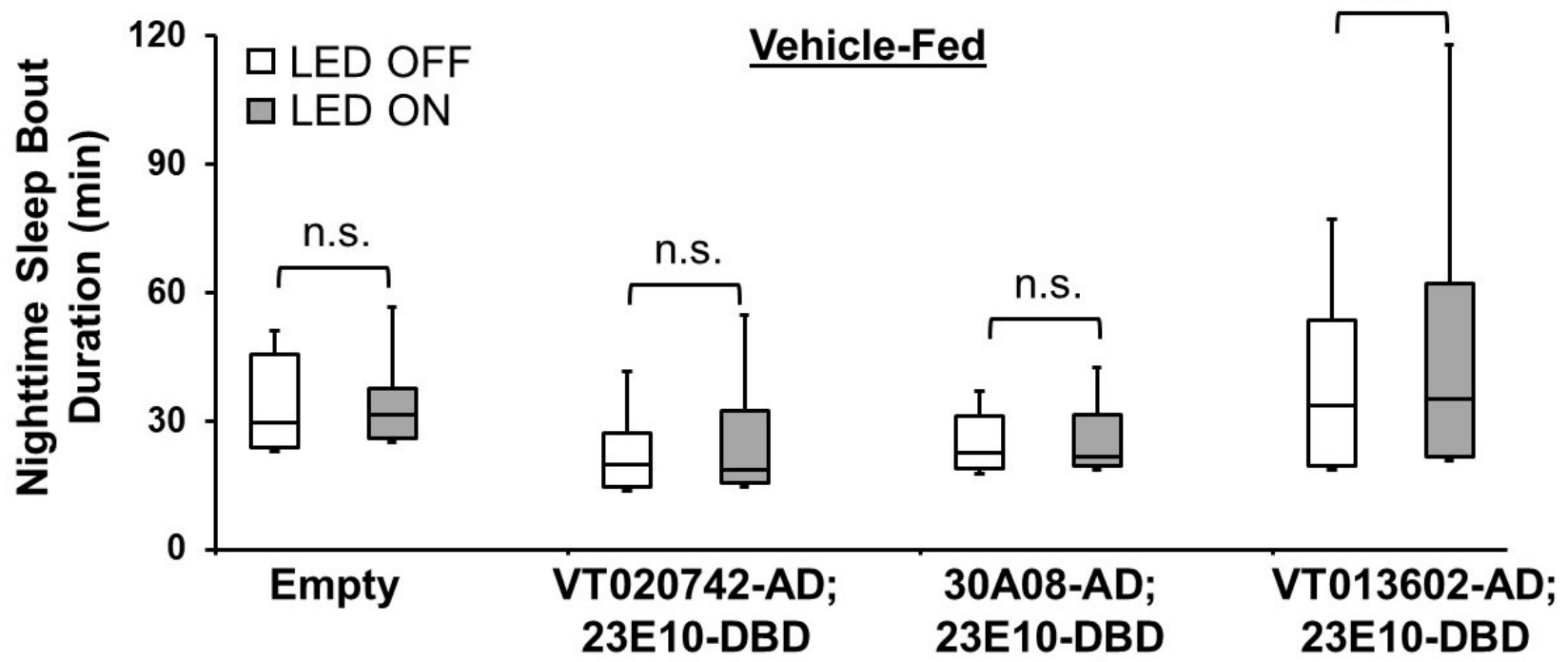


bioRxiv preprint doi: https://doi.org/10.1101/2022.02.02.478882; this version posted February 9,2022 . The copyright holder for this preprint (which was not certified by peer review) is the author/funder, who has granted bioRxiv a license to display the preprint in perpetuity. It is made available under aCC-BY-NC-ND 4.0 International license.

Figure 4-S4

A VT020742-AD; 23E10-DBD>UAS-mCD8RFP; 30A08-LexA> LexAop2mCD8GFP
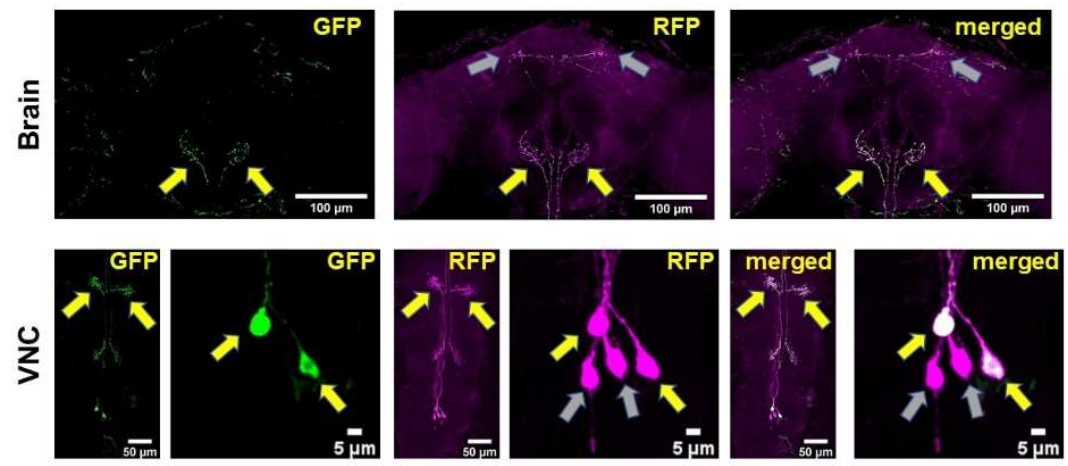

B VT020742-AD; 23E10-DBD>UAS-GFP; 30A08-LexA> LexAop2-KZip+
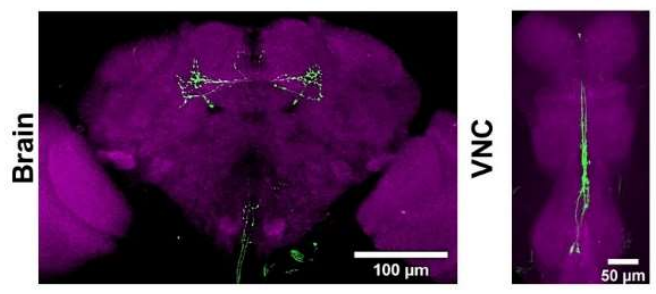

C
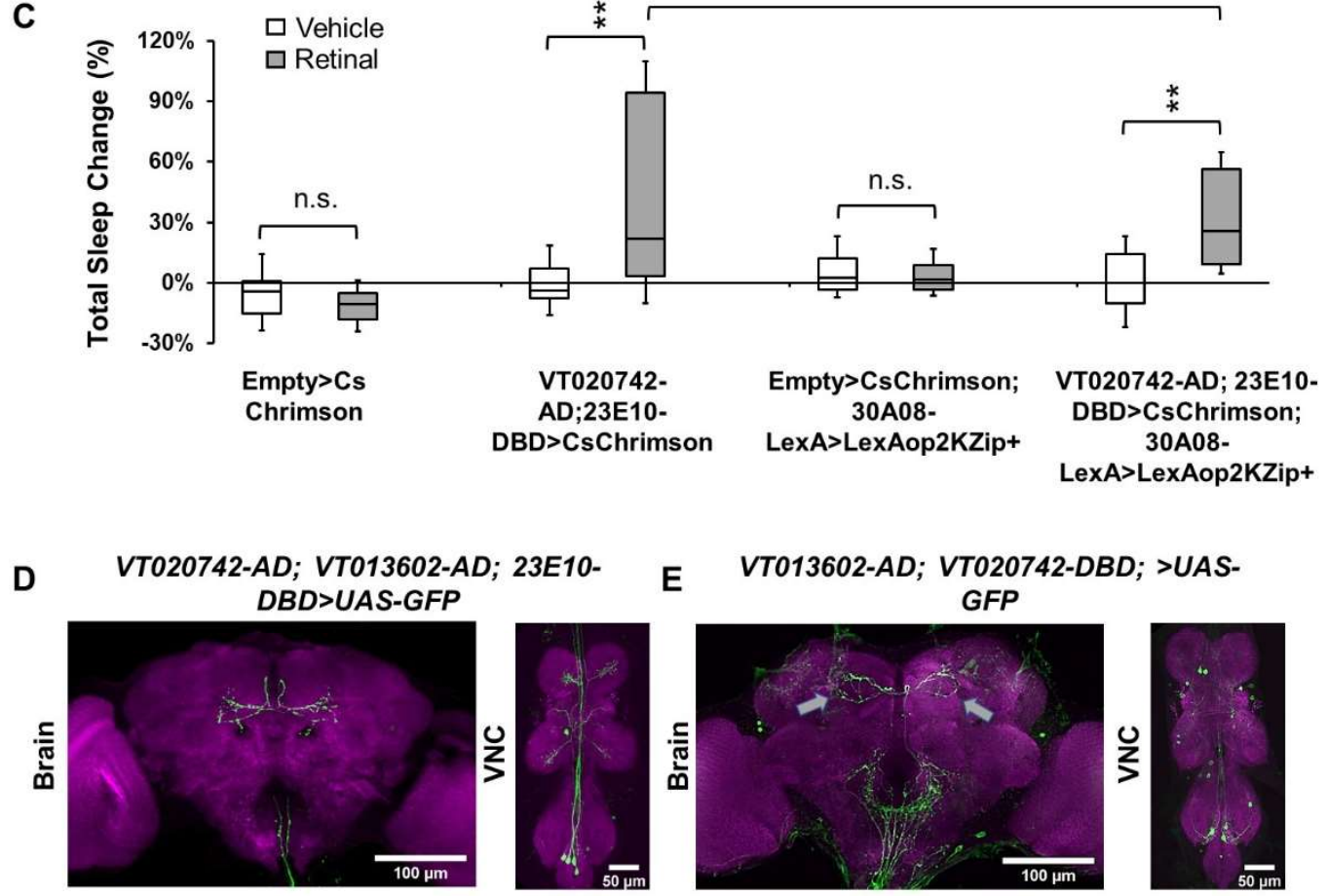

; >UAS-

F

Empty> UAS-TrpA1

$$
\text { G }
$$

VT013602-AD; VT020742DBD> UAS-TrpA1
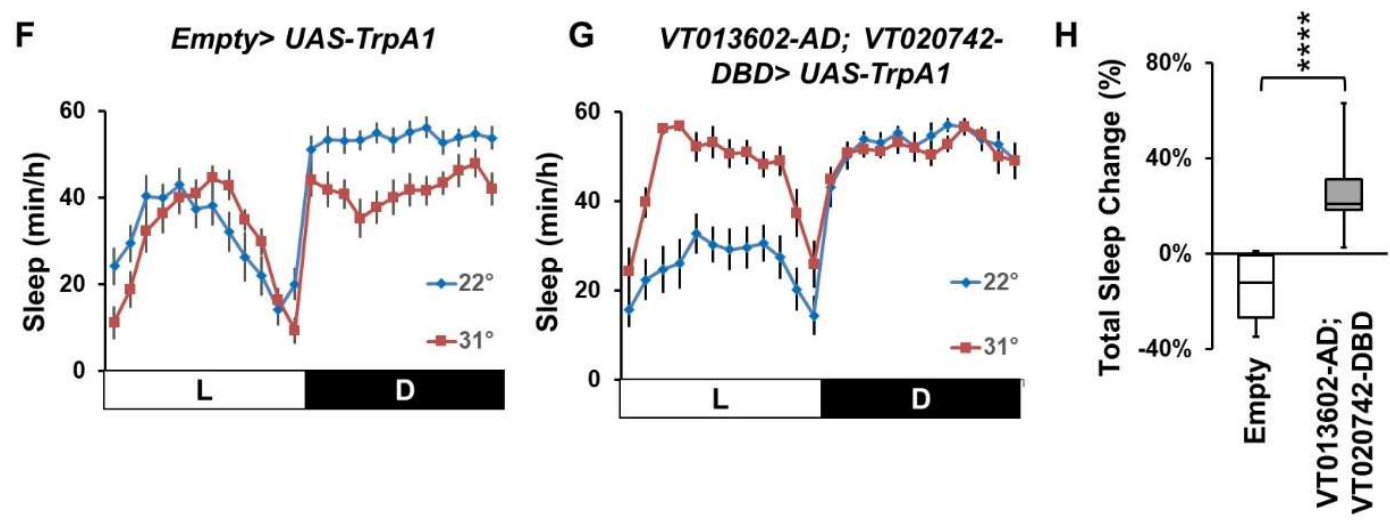
Figure 4-S5

\section{VT013602-AD; 23E10-DBD>UAS-CsChrimson}

\section{Retinal-Fed}

$\underline{\text { Vehicle-Fed }}$

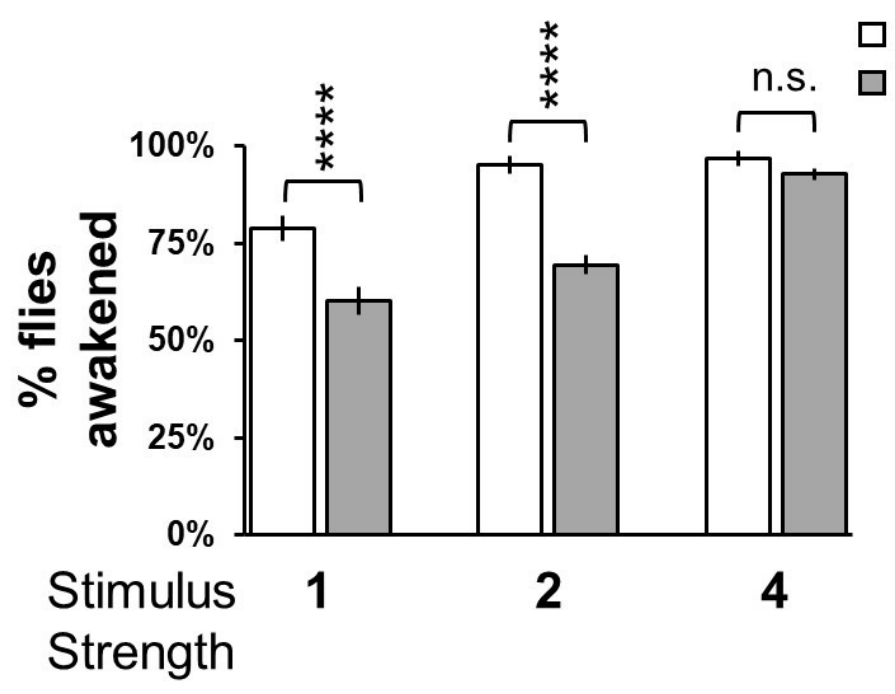

$\square$ LED OFF

LED ON

원

$100 \%$

] n.s.

n.s.

n.s.

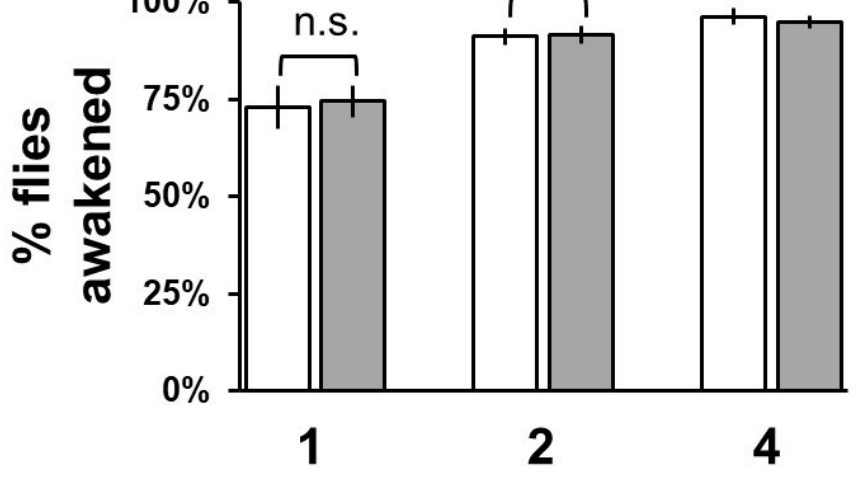


bioRxiv preprint doi: https://doi.org/10.1101/2022.02.02.478882; this version posted February 9, 2022. The copyright holder for this preprint (which was not certified by peer review) is the author/funder, who has granted bioRxiv a license to display the preprint in perpetuity. It is made available under aCC-BY-NC-ND 4.0 International license.

\section{Figure 6-S1}

A

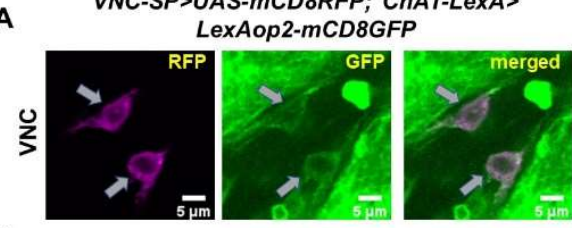

B

23E10-GAL4>UAS-mCD8RFP; ChAT-LeXA>

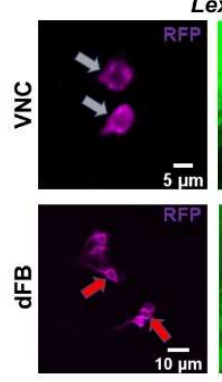

C

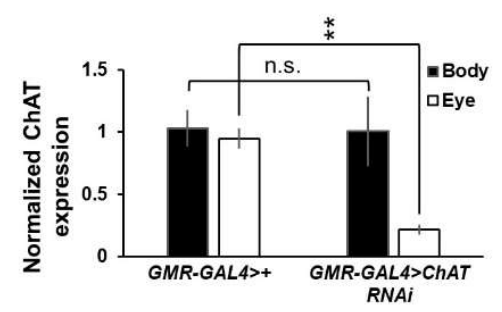

F
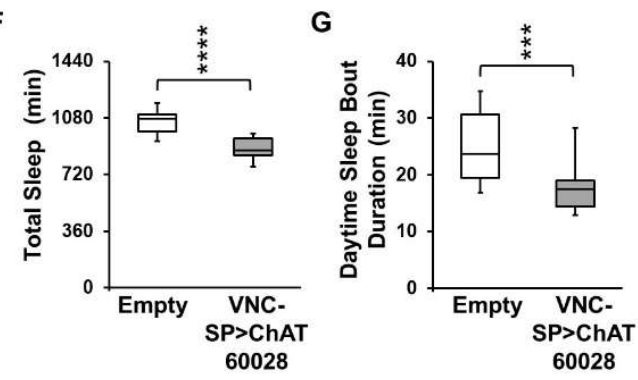

J

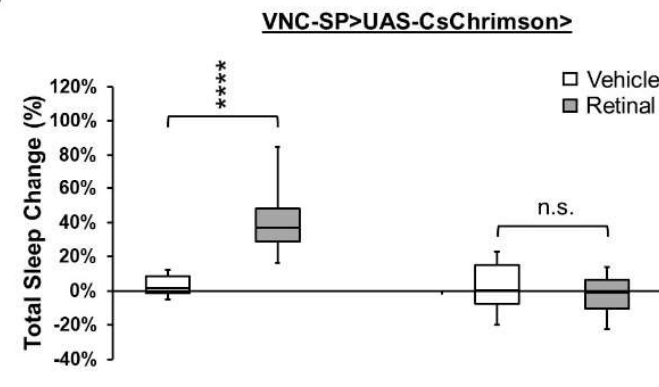

2x LexAop2-KZip+

ChAT-LexA> 2x LexAop2KZip+

L

$\underline{X}>$ UAS-CsChrimson; ChAT-LexA>LexAop2$\underline{\text { KZip }+}$

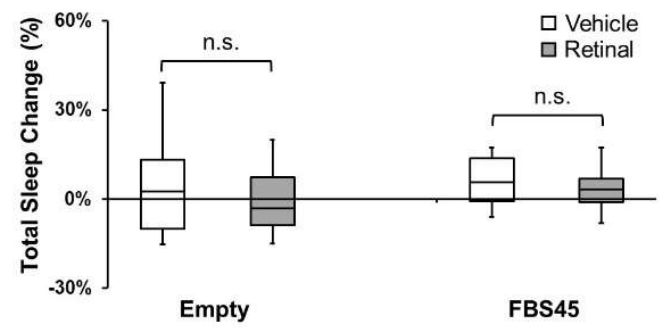

E

H

M
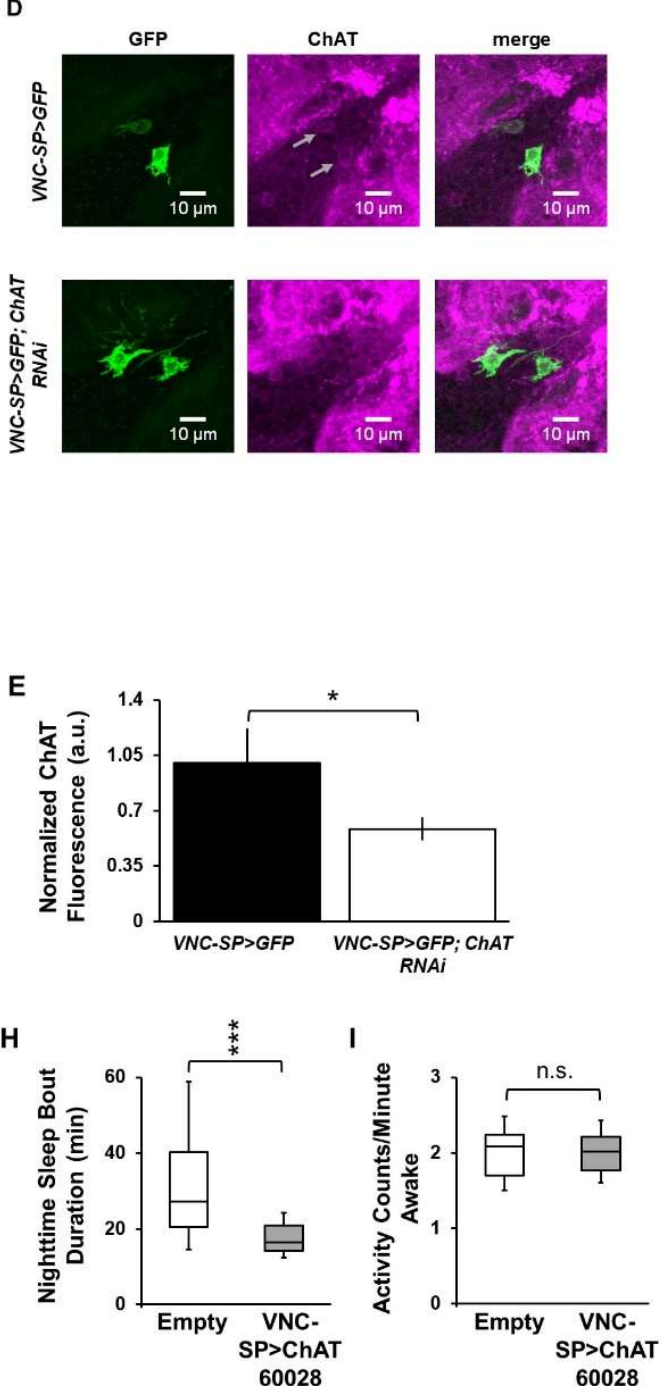

K

VNC-SP>UAS-GFP

VNC-SP>UAS-GFP;

ChAT-LexA $>2 x$

LexAop2-KZip+

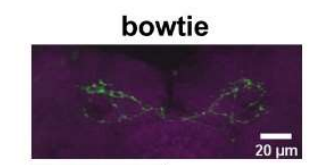

bowtie
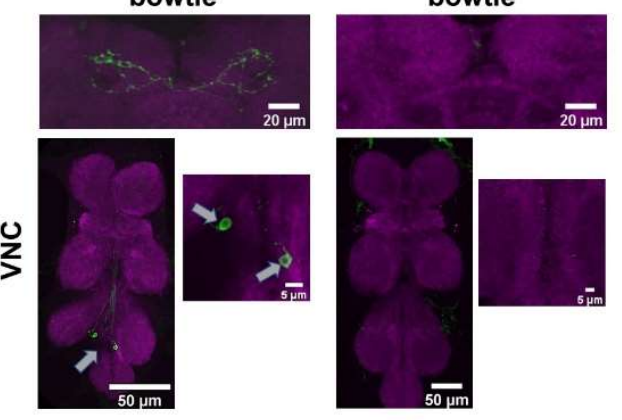

FBS45>UAS-GFP; ChAT-LexA>

LexAop2-KZip+

bowtie

bowtie
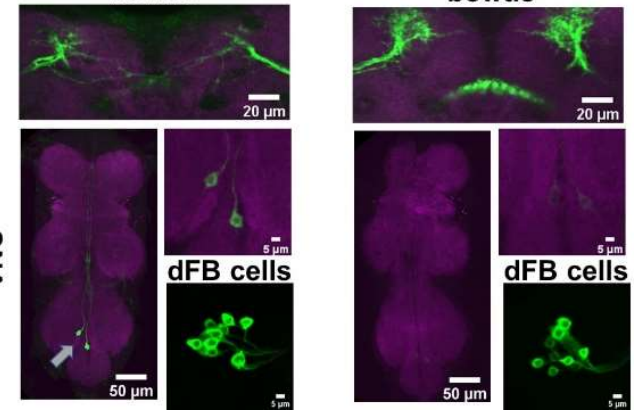
Figure 6-S2

\section{VNC-SP>UAS-5XDenMark-V5; UAS-syt.eGFP}
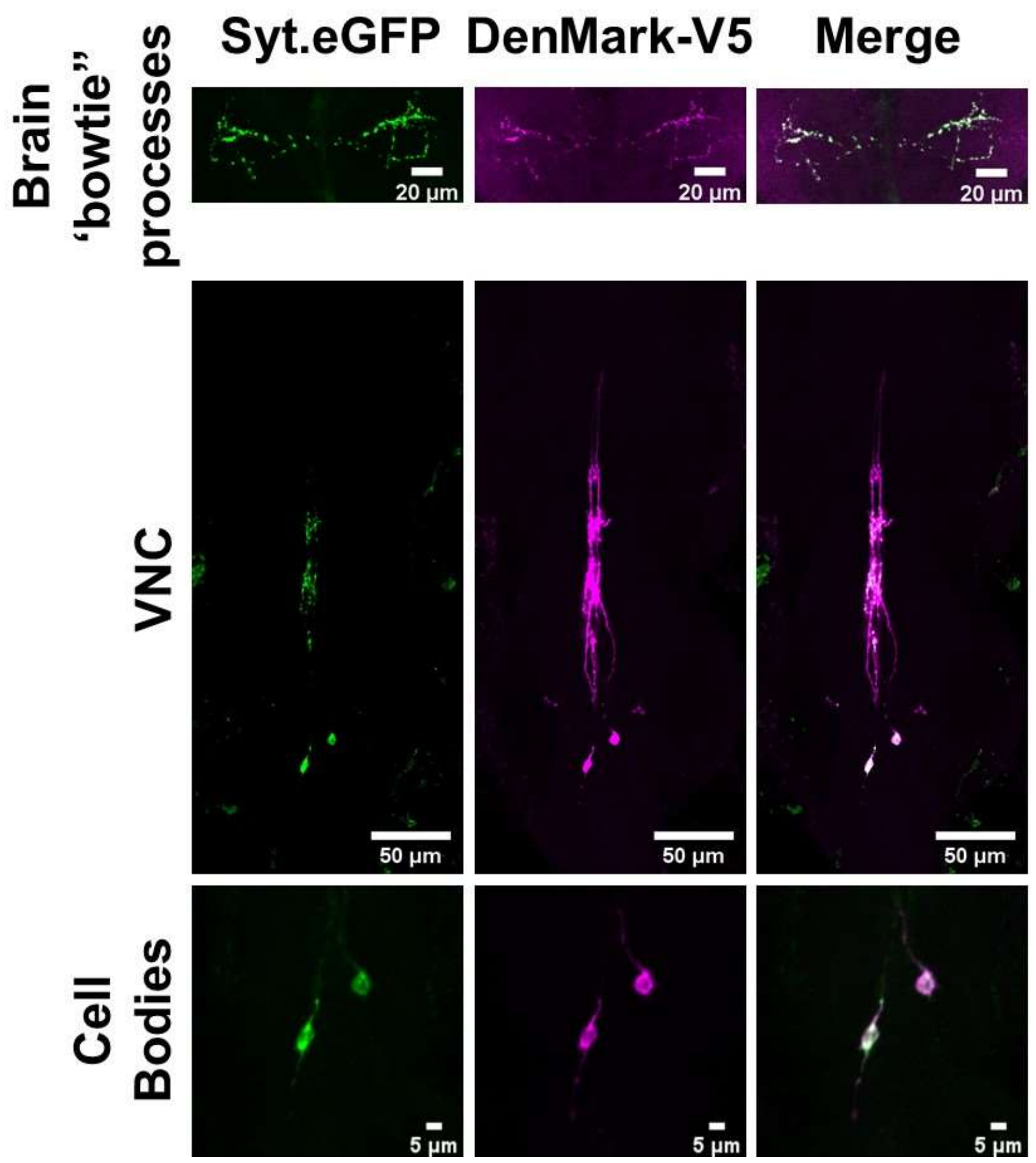